\title{
Optimal control of flow with discontinuities
}

\author{
Chris Homescu and I.M. Navon* \\ Department of Mathematics and \\ School of Computational Science and Information Technology \\ Florida State University \\ Tallahassee FL 32306
}

\begin{abstract}
Optimal control of the 1-D Riemann problem of Euler equations whose solution is characterized by discontinuities is carried out by both nonsmooth and smooth optimization methods. By matching a desired flow to the numerical model for a given time window we effectively change the location of discontinuities. The control parameters are chosen to be the initial values for pressure and density fields. Existence of solutions for the optimal control problem is proven. A high resolution model and a model with artificial viscosity, implementing two different numerical methods, are used to solve the forward model. The cost functional is the weighted difference between the numerical values and the observations for pressure, density and velocity. The observations are constructed from the analytical solution. We consider either distributed observations in time or observations calculated at the end of the assimilation window. We consider two different time horizons and two sets of observations. The gradient (respectively a subgradient) of the cost functional, obtained from the adjoint of the discrete forward model, are employed for the smooth minimization (respectively for the nonsmooth minimization) algorithm. Discontinuity detection improves the performance of the minimizer for the model with artificial viscosity by selecting the points where the shock occurs (and these points are then removed from
\end{abstract}


the cost functional and its gradient). The numerical flow obtained with the optimal initial conditions obtained from the nonsmooth minimization matches very well the observations. The algorithm for smooth minimization converges for the shorter time horizon but fails to perform satisfactorily for the longer time horizon.

Key words: optimal control of shocked flow, adjoint method, nonsmooth optimization, LBFGS unconstrained minimization, Euler equations, discontinuity detection, distributed observations

* Corresponding author.

Email addresses: chome@math.fsu.edu (Chris Homescu), navon@csit.fsu.edu

(I.M. Navon).

$U R L:$ http://www.csit.fsu.edu/ navon (I.M. Navon). 


\section{Introduction}

Optimal control methods are presently employed for various applications in different fields: e.g. aerodynamics, meteorology, acoustics, chemistry, economical models to mention but a few.

The vast majority of applications consider the minimization of a smooth cost functional. We consider the case of a nonsmooth cost function and the application of the adjoint method for minimizing this cost functional.

Recently optimal control involving nonsmooth functions has attracted the attention of an increasing number of researchers due to availability of new methods of nondifferentiable optimization employing subgradients following the seminal work of Lemarechal [37] (e.g. Lemarechal [38], Bonnans et al. [7], Schramm and Zowe [58], Luksan and Vlcek [45], Makela and Neittaanmaki [47] to cite but a few).

Nonsmooth cost functionals were employed in variational data assimilation in atmospheric sciences (Zhang et al. [71]), for inverse design problems involving transonic diffusers : 1-D (Narducci et al. [52] or 2-D (Dadone et al. [13]), in acoustics (Habbal [26]), for the research of a convex hull with bounded curvature of a given set of points (Hassold [27]), in mechanical structures (minimizing the maximal stress over an arch structure Habbal [25]), for chromatography (James and Sepulveda [34]), capital asset management (Leonard and Long [?]), in the design of a duct flow with a shock (Frank and Shubin [17], Cliff et al. [11], Iollo et al. [32]), for airfoil design (Jameson [35], Matsuzawa and Hafez [50], Iollo and Salas [31] and Giles and Pierce [20]). 
The presence of discontinuities creates serious theoretical and numerical difficulties. Good shock-capturing schemes with low continuity properties often cannot be combined successfully with efficient optimization methods requiring smooth functions (e.g. gradient-based methods). To alleviate this problem one can use methods that are relatively insensitive to the non smoothness of the cost function. Stochastic optimization methods are applied for the design of a minimum time changeover operation for a pressure vessel avoiding the formation of explosive mixtures (Barton et al. [3]) or for aerodynamic shape optimization (Huyse and Lewis [30]). Genetic algorithms (Oyama et al. [56]) were also used for wing optimization. For these non-gradient-based methods the drawback is the relatively large number of analyses required (i.e. large memory demands) as the number of variables increases.

In the case of gradient-based methods different remedies to allevaiate the influence of the discontinuities were employed. For variables which are continuous across the shock one can avoid dealing with shocks by considering cost functions based on the above variables (e.g. the surface flux for inverse nozzle design as used by Matsuzawa and Hafez [49]). For most cases the shocks were smoothed using numerical dissipation. It was shown that sometimes smoothing is equivalent to modifying the cost function (Matsuzawa and Hafez [49]). An alternative smoothing procedure has been introduced by Valorani and Dadone [63], namely a filtering process which was obtained by modifying a set of sensitivity equations through by adding artificial dissipative terms. The optimization search was performed on original nonsmooth objective function computed with an accurate (non smoothed) flow analysis but with smoothed flow sensitivities.

If the shocks are weak at design conditions (like in the case of transonic flows) 
acceptable results can be obtained by addition of artificial dissipation. However, accurate treatment of the shock waves is essential in other cases (e.g. supersonic flows). The alternative approach to shock smearing is shock fitting which involves careful integration of the objective function through the shock wave (Narducci et al. [52]). Perturbation of a discontinuous function produces delta functions and formulations based on variations of smooth functions have to be modified (Iollo et al. [32]). Another approach was to introduce the shock location as an explicit control variable (Cliff et al. [11]). A coordinate straining method was also employed by Narducci et al. [52]. It consists of a coordinate transformation aimed at aligning the calculated shock with the target followed by addition of a penalty term proportional to the square of distance between the shocks.

Results for the optimal control of the Euler equations were obtained, among others, by Anderson and Venkatakrishnan [1] (in 2-D), Arian and Salas [2] (in 2-D), Dadone and Grossman [14],[15] (2-D and 3-D), Cliff et al. [10],[9],[11] (1-D and 3-D).

Theoretical contributions (combined with practical applications in certain cases) for the adjoint method were provided by Giles and Pierce [18],[19],[20],[21] (for Euler equations) and Ulbrich [64],[65], [67], [66] (in the setting of optimal control for scalar conservation laws). A generalized adjoint for physical processes in atmospheric sciences with parameterized discontinuities was studied by $\mathrm{Xu}[70]$. Numerical aspects of the adjoint model for discontinuous nonlinear atmospheric models were discussed by Zhang et al. [72].

Problems with discontinuities in an optimal control setting or in sensitivitybased control were studied by Mohammadi and Pironneau [51], Gunzburger 
[24], [23], Tolsma and Barton [62] and Zhang et al. [71].

Practical aspects of control of problems with shocks were presented by Iollo and Salas [33], Birkmeyer et al. [5], Stanewsky [61], Jameson [35], Bein et al. [4] or Wang et al. [69].

This article presents theoretical and numerical results for an optimal control of the unsteady 1-D Riemann problem of Euler equations. The numerical solutions of the optimal control problem were obtained using both nonsmooth and smooth optimization algorithms. The present research extends our previous results in optimal control for a smooth cost functional (Homescu et al. [28]) and sensitivity analysis for discontinuous flow (Homescu and Navon [29]) with optimization methods suitable for discontinuous cost functionals.

This specific problem was chosen due to the fact that it has an analytical solution which is characterized by the presence of many types of discontinuities: shocks, contact discontinuities and wave rarefaction regions. This Riemann problem may be briefly described in the following way: a gas tube is divided by a membrane into two regions with different values for pressure and density fields and a zero velocity field. After the membrane is suddenly removed the gas moves freely.

Our optimal control problem has very interesting aerodynamic applications and consists of moving the regions of discontinuities to desired locations by matching the desired flow to the numerical flow. The control parameters consist of the initial values of pressure and density to the left and to the right of the membrane. We consider the initial velocity to the left and to the right of themembrane to be zero. The cost functional is the weighted difference between the observations and the numerical values for density, pressure and 
velocity fields. The observations obtained from the analytical solution of the Riemann problem are computed in two ways: either at the end of the assimilation window or distributed in time during the assimilation window.

Two numerical models were chosen, representative for possible approaches of solving a flow with discontinuities: a high-resolution model (HRM) and a model with artificial viscosity (AVM).

We employ a nonsmooth optimization algorithm described in Luksan and Vlcek [45], [46], Vlcek and Luksan [68], which is a hybrid algorithm that combines the characteristics of the variable metric method and the bundle method. We also apply a smooth optimization algorithm (L-BFGS) described in Nocedal [54] and Liu and Nocedal [43]). Both methods require the computation of a subgradient (respectively the gradient) of the cost functional. This subgradient (respectively gradient) is obtained from the adjoint model derived from the original numerical model. Accuracy tests for both the gradient and subgradient obtained via the adjoint method.

We consider two time horizons which are representative for the time evolution of the flow. Their length was chosen for two main reasons. First we wanted to ensure that all desired characteristics of the discontinuities are still present in the flow at the end of each time window. Second, we selected the larger time window such that if we were to slightly increase it some of the discontinuity characteristics will disappear.

We obtained excellent results using nonsmooth optimization for both models and for both time horizons. The numerical flow corresponding to the optimized initial conditions matches closely the observations and one can see from the Figures presented that the location of the discontinuities was changed to 
changed to the desired location.

The L-BFGS algorithm did not converge in many cases. Even for the cases where convergence was obtained one may notice that there is a large difference between the L-BFGS optimization results and the desired values of the control parameters.

For the model with artificial viscosity a discontinuity detection method was used to eliminate the points where the shock is located from the computation of the cost functional and its gradient (or subgradient). As a result of this technique the optimized results were obtained at the same level of accuracy but in fewer minimization iterations.

The article is organized as follows. Section 2 introduces the governing equations (unsteady Euler 1-D) for the flow including its analytical solution. Section 3 describes the discretization of the 1-D Riemann problem in space and time using both the high-resolution model and the artificial viscosity model. In section 4 we present results related to existence of solutions for optimal control problem considered. Section 5 describes the methods of nonsmooth and smooth unconstrained optimization employed for this research. Methods of detection of discontinuities in data are presented in section 6 . The adjoint method for computing the gradient (or a subgradient) of the cost functional with respect to the control parameters is presented in Appendix A. Procedures for validating the adjoint code and checking the accuracy of the gradient (subgradient) computed using the adjoint model are also presented in Appendix A. Numerical results obtained for the optimal control problem of 1-D Euler equations are displayed in the Tables and Figures of section 7. Section 7 also discusses the evolution of the cost functional for both methods of mini- 
mization, both models and both time horizons considered. Finally section 8 presents the summary and conclusions. 


\section{Governing equations}

The one-dimensional unsteady equations of gas dynamics (Euler equations) can be written in conservation law form as:

$$
\mathbf{U}_{t}+\mathbf{F}(\mathbf{U})_{x}=0
$$

where

$$
\mathbf{U}=\left[\begin{array}{c}
\rho \\
m \\
e
\end{array}\right], \quad \mathbf{F}(\mathbf{U})=\left[\begin{array}{c}
m \\
\frac{m^{2}}{\rho}+P \\
\left(\frac{m}{\rho}\right)(e+P)
\end{array}\right]
$$

$\rho$ is the density, $u$ is the velocity, $m=\rho u$ is momentum, $P$ is the pressure and $e$ is the internal energy per unit volume. The variables are related by $e=\rho \varepsilon+\frac{1}{2} \rho u^{2}$, where $\varepsilon=\frac{P}{(\gamma-1) \rho}$ is the internal energy per internal mass with $\gamma$ the ratio of specific heats (which is taken to be 1.4).

The "shock-tube problem" can be described in the following way: a tube, filled with gas, is initially divided by a membrane into two sections. The gas has a higher density and pressure in one half of the tube than in the other half, with zero velocity everywhere. The initial conditions for density, velocity and pressure are similar to the values for the Sod shock-tube problem [60]:

$$
\rho_{\text {left }}=1.0>\rho_{\text {right }}=0.125, \quad u_{\text {left }}=u_{\text {right }}=0.0, \quad p_{\text {left }}=1.0>p_{\text {right }}=0.1
$$

where the subscripts left and right correspond to the initial position with respect to the membrane. At time $t=0$ the membrane is suddenly removed and the gas is allowed to flow. We expect a net motion in the direction of lower 
pressure. Assuming the flow is uniform across the tube, there is variation in only one direction and the 1-D Euler equations apply. One should calculate the flow variables: pressure, density and velocity as a function of time and space.

The solution of this Riemann problem for Euler equations consists of 5 distinct regions (see Figures 3 and 4). The description of these regions follows with the corresponding region index in the parentheses: low pressure and density region (region 1), area between shock and contact discontinuity (region 2), area between contact discontinuity and rarefaction wave (region 3), rarefaction wave region (region $\mathrm{R}$ ), high pressure and density region (region 4).

The exact solution can be found explicitly as a function of $x$ and $t$ (Liepmann and Roshko [42]). It is given by the following equations with indices $1,2,3,4, R$ related to the corresponding 5 regions described above. 


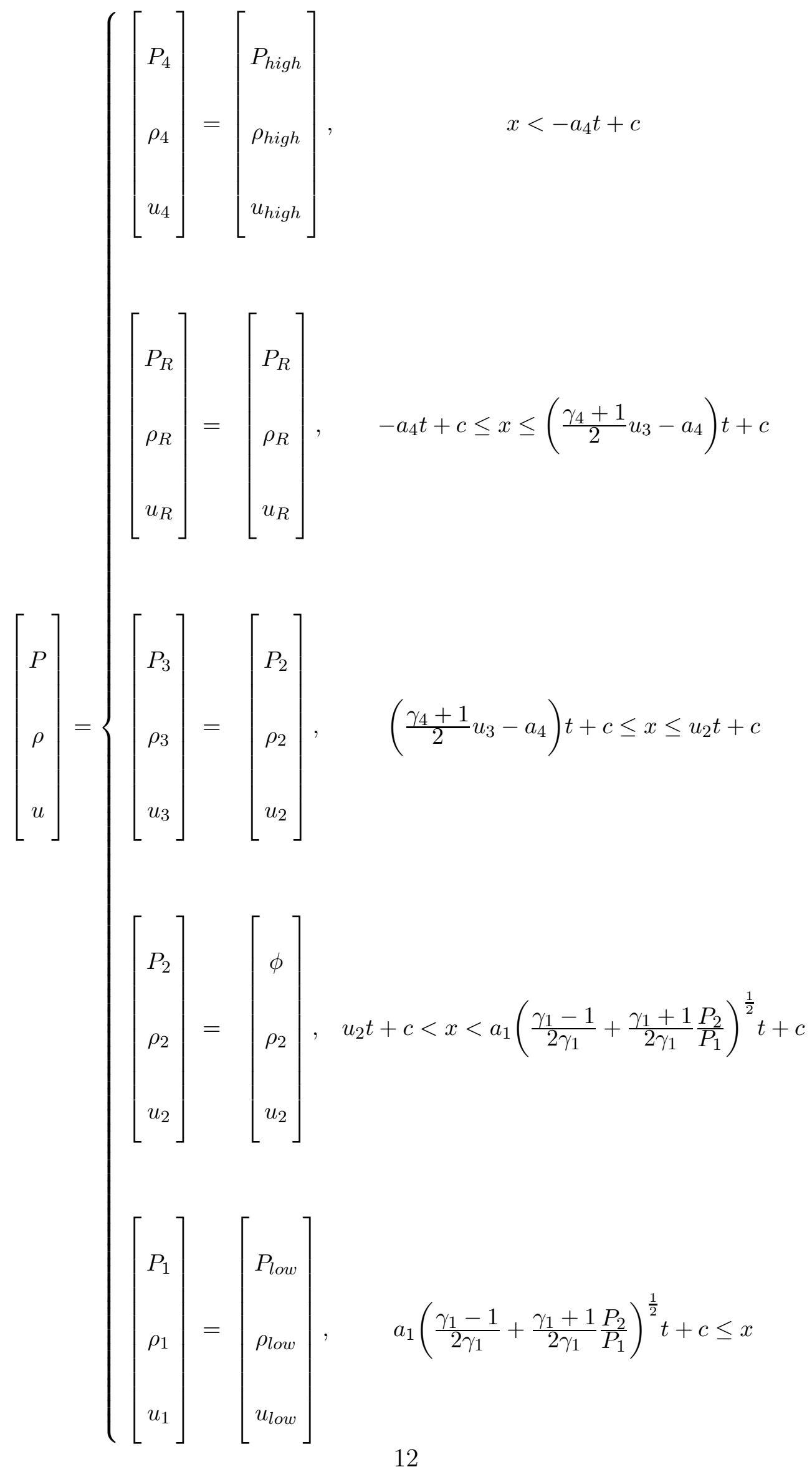


where $a_{i}^{2}=\frac{\gamma_{i} P_{i}}{\rho_{i}}, \gamma_{i}=\gamma$ for $i=1, \ldots, 4$

and where $\phi$ is given implicitly by

$$
\frac{P_{4}}{P_{1}}=\frac{\phi}{P_{1}}\left(1-\frac{\left(\gamma_{4}-1\right)\left(a_{1} / a_{4}\right)\left(\phi / P_{1}-1\right)}{\sqrt{2 \gamma_{1}} \sqrt{2 \gamma_{1}+\left(\gamma_{1}+1\right)\left(\phi / P_{1}-1\right.}}\right)^{\frac{-2 \gamma_{4}}{\gamma_{4}-1}}
$$

The remaining variables : $\rho_{2}, u_{2}$ and $\rho_{3}$ are given by

$$
\begin{array}{r}
\rho_{2}=\rho_{1} \frac{P_{2}}{P_{1}}\left(\frac{1+\frac{\gamma_{1}-1}{\gamma_{1}+1} \frac{P_{1}}{P_{2}}}{1+\frac{\gamma_{1}-1}{\gamma_{1}+1} \frac{P_{2}}{P_{1}}}\right) \\
u_{2}=a_{1}\left(\frac{P_{2}}{P_{1}}-1\right) \sqrt{\frac{2 \gamma_{1}}{\left(\gamma_{1}+1\right) \frac{P_{2}}{P_{1}}+\left(\gamma_{1}-1\right)}} \\
\rho_{3}=\rho_{4}\left(\frac{P_{3}}{P_{4}}\right)^{\frac{1}{\gamma_{4}}}
\end{array}
$$

and, in the rarefaction wave, the quantities $P_{R}, \rho_{R}$ and $u_{R}$ are given by

$$
\begin{array}{r}
P_{R}=P_{4}\left(1-\frac{\gamma_{4}-1}{2} \frac{u_{R}}{a_{4}}\right)^{\frac{2 \gamma_{4}}{\gamma_{4}-1}} \\
\rho_{R}=\rho_{4}\left(1-\frac{\gamma_{4}-1}{2} \frac{u_{R}}{a_{4}}\right)^{\frac{2}{\gamma_{4}-1}} \\
u_{R}=\left(\frac{u_{3}-u_{4}}{\frac{\gamma_{4}+1}{2} u_{3}}\right)\left(\frac{x-c}{t}\right)+\frac{a_{4} u_{3}-\left(a_{4}-\frac{\gamma+4+1}{2} u_{3}\right) u_{4}}{\frac{\gamma_{4}+1}{2} u_{3}}
\end{array}
$$

The subscripts for the variables $u_{2}, p_{R}$, etc match the corresponding region of the solution in which they are located (e.g. $p_{R}$ is the value of the pressure in the rarefaction region). 


\section{$3 \quad$ Numerical models}

The main difficulties encountered when solving numerically the shock-tube problem of gas dynamics (and, in general, for any problem which has a nonsmooth solution) appear in the regions of discontinuities. The numerical solution may be smoothed in those regions (e.g. due to introduction of a dissipation term) or it can sharpen discontinuities (using high-resolution methods). For this reason we chose one numerical model from each of the above mentioned categories: namely a model with artificial viscosity (AVM) and a high-resolution model (HRM with a Riemann solver.

As a footnote we mention that for very accurate numerical solutions adaptive mesh refinement AMR may be used in conjunction with Riemann solvers (e.g. Leveque [41] for Euler equations). Our experience with a model AMR in the framework of sensitivity analysis for discontinuous flows was presented in Homescu and Navon [29].

The main goal of our research is to perform optimal control using either smooth or nonsmooth optimization techniques for minimizing the cost functional. The minimization requires availability of either the gradient or the subgradients of the said cost functional obtained using the adjoint model derived from the forward model (either AVM or HRM models).

The first model (by T.J. Cowan [12]) uses finite elements which are piecewise constant in time and piecewise linear in space. The elements are discontinuous in time but continuous in space. By using discontinuous discretization in time we were able to march sequentially through time and solve for only a fraction of the total solution at one time. To improve the stability of the method a least- 
squares operator is added to the basic Galerkin formulation. In order to obtain non-oscillatory approximations to discontinuities discontinuity-capturing operators have been developed within the framework of this Galerkin/least squares method. For more details about this modified discontinuous Galerkin method the reader is referred to Shakib et al. [59].

An artificial viscosity term was included to stabilize the numerical solution and it has the effect of spreading flow discontinuities over several computational cells. The method employs a high-order scheme for the smooth regions of the flow combined with a low-order solution which is employed near the discontinuities. The above combination is described in Lohner et al. [44] as MC-ML method (with the high-order scheme using a consistent mass matrix while the low-order scheme employs a lumped mass matrix).

We describe below the second model (using a code from the package CLAWPACK written by R. Leveque [40], [41]) which employs Roe's approximate Riemann solver combined with an entropy fix.

Consider a standard form of a homogeneous conservation law:

$$
q_{t}(x, t)+f(q(x, t))_{x}=0
$$

The basic algorithm depends on a Riemann solver that, for each set of data $\left(q^{L}, q^{R}\right)$ returns a set of $M_{w}$ waves $W^{p}$ and speeds $\lambda^{p}$ satisfying

$$
\sum_{p=1}^{M_{w}} \mathcal{W}^{p}=q^{R}-q^{L} \equiv \Delta q
$$

It also returns the left-going and right-going flux differences $\mathcal{A}^{-} \Delta q$ and $\mathcal{A}^{+} \Delta q$ 
which satisfy the relationship:

$$
\mathcal{A}^{-} \Delta q+\mathcal{A}^{+} \Delta q=f\left(q^{R}\right)-f\left(q^{L}\right)
$$

The Roe solver employed here consists of solving a particular linear system

$$
q_{t}+A_{i} q_{x}=0
$$

where $A_{i}$ is the Roe matrix depending on data $\left(q_{i-1}, q_{i}\right)$. The solution consists of waves of the form $\mathcal{W}_{i}^{p}=\lambda_{i}^{p} r_{i}^{p}$ where $r_{i}^{p}$ is the $p$-th eigenvector of $A_{i}$, which propagate with speeds $\lambda_{i}^{p}$, the corresponding eigenvalue of $A_{i}$. The flux differences are defined as

$$
\begin{aligned}
\mathcal{A}^{+} \Delta q_{i} & =\sum_{\lambda_{i}^{p}>0} \lambda_{i}^{p} \mathcal{W}_{i}^{p} \\
\mathcal{A}^{-} \Delta q_{i} & =\sum_{\lambda_{i}^{p}<0} \lambda_{i}^{p} \mathcal{W}_{i}^{p}
\end{aligned}
$$

Since the analytical solution includes a rarefaction wave, an entropy fix which modifies the flux differences (without changing the waves) was included (otherwise the Roe linearization violates the entropy condition). 


\section{Theoretical setting of the optimal control problem}

We solve the following optimal control problem

minimize the cost functional $\mathbf{J}(q, z)$ subject to $z \in \mathcal{U}_{\text {ad }}$

(OPT)

where $z$ is the control, $\mathcal{U}_{a d}$ is the space of adnissible controls and $q=q(z)$ is the entropy solution of the system of conservation laws (Euler 1-D equations described in the previous section):

$$
\begin{aligned}
& \frac{\partial q}{\partial t}+\frac{\partial F(q)}{\partial x}=0 \\
& q(x, 0)=z(x)
\end{aligned}
$$

with $0 \leq x \leq 1$ and $0 \leq t \leq T_{W}$ ( $T_{W}$ being the length of the assimilation window).

Since the solution of the system (13) may develop discontinuities after a finite time weak solutions should be considered. An additional entropy condition must be imposed to select the "physically" relevant weak solution.

An entropy function $\mathbf{E F}$ is defined for which an additional conservation law holds for smooth solutions that becomes an inequality for discontinuous solutions (Leveque [40], Godlewski and Raviart [22]). For the Euler equations of gas dynamics (which are employed for this research) there exists a physical quantity called the entropy known to be constant along particle paths in smooth flow and to jump to higher value as the gas crosses a shock. The correct weak solution is picked out using a property of the entropy, namely that it can never jump to a lower value (this approach was employed for the high resolution model described in the previous section). 
For the system of gas dynamics equations, which is a strictly hyperbolic symmetrizable nonlinear system of conservation laws, entropy functions can be found (e.g. Godlewski and Raviart [22], Leveque [40] ).

We introduce the definition of the entropy solution (Godlewski and Raviart $[22]):$

A weak solution $q$ of (13)-(14) is called an entropy solution if q satisfies, for all entropy functions $\mathbf{E F}$ of (13) and for all test functions $\phi \in C_{0}^{1}([0,1] \times[0, \infty))$, $\phi \geq 0$,

$$
\int_{t=0}^{\infty} \int_{x=0}^{x=1}\left(\mathbf{E F}(q) \frac{\partial \phi}{\partial t}+F(q) \frac{\partial \phi}{\partial x}\right) d x d t+\int_{x=0}^{x=1} \mathbf{E F}(z(x)) \phi(x, 0) d x \geq 0
$$

We will follow here the approach of Ulbrich [66] to derive existence results for optimal controls. His work, related to scalar laws of conservation with source terms, can be extended to our case (the 1-D system of Euler equations without source terms).

For our problem the control vector $z(x)$ is:

$$
z(x)=\left[\begin{array}{c}
\rho(x, 0) \\
m(x, 0) \\
e(x, 0)
\end{array}\right]=\left[\begin{array}{c}
\rho(x, 0) \\
\rho(x, 0) u(x, 0) \\
\frac{P(x, 0)}{(\gamma-1)}+\frac{1}{2} \rho(x, 0)(u(x, 0))^{2}
\end{array}\right]
$$

with $\rho$ the density, $u$ the velocity, $m=\rho u, P$ the pressure and $\gamma$ the ratio of specific heats.

Since $z$ is obtained using the bounded initial values of the pressure, velocity and density we may consider that the controls are in $\left(L^{\infty}[0,1]\right)^{3}$. If the control 
problem (OPT) is particularized to the optimal control prblem for 1-D Euler equations for gas dynamics (described in Section 2) the existence of the optimal controls is obtained as a consequence of four properties derived below.

(P1) The function $F$, which appears in the system of conservation laws (13), is locally Lipschitz.

(P2) The admissible set $\mathcal{U}_{a d}$ is bounded in $\left(L^{\infty}[0,1]\right)^{3}$ and closed in $\left(L_{l o c}^{1}([0,1])^{3}\right.$.

(P3) If we denote by $B V((0,1))$ the space of functions of bounded variations on the interval $(0,1)$ and using the fact that the embedding $(B V(\Omega))^{3}->$ $\left(L^{1}(\Omega)\right)^{3}$ is compact for any open bounded set $\Omega \subset(0,1)$ we obtain that $\mathcal{U}_{a d}$ is compact in $\left(L_{l o c}^{1}[0,1]\right)^{3}$.

In our research the cost functional $\mathbf{J}$ for the optimal control problem (OPT) assumes two possible forms.

The first expression of the cost functional is

$$
\mathbf{J}(q, z)=\int_{0}^{1}\left(q\left(x, T_{W}\right)-q^{o b s}\left(x, T_{W}\right)\right)^{2} d x
$$

with $q^{o b s} \in L^{\infty}([0,1])$ the observations distributed at assimilation time $T_{W}$.

The second cost functional is defined as

$$
\mathbf{J}(q, z)=\int_{t=0}^{t=T_{W}} \int_{x=0}^{x=1}\left(q(x, t)-q^{o b s}(x, t)\right)^{2} d x d t
$$

with $q^{\text {obs }} \in\left(L^{\infty}\left([0,1] \times\left(0, T_{W}\right)\right)\right)^{3}$ the observations at assimilation times $0 \leq t \leq T_{W}$ 
For both forms: (16), (17) we have the following property:

(P4) The cost functional $\mathbf{J}$ is (at least) sequentially lower semicontinuous.

Using the properties (P1)-(P4) one can prove that the optimal control problem (OPT) has a solution $\hat{z} \in \mathcal{U}_{a d}$ in a similar way to the proof of existence of optimal controls obtained by Ulbrich [66].

First we prove that if $\mathbf{J}$ satisfies $\mathrm{P} 4$ ) then

$$
z \in\left(\mathcal{U}_{a d} \subset\left(L_{l o c}^{1}[0,1]\right)^{3}\right) \hookrightarrow \mathbf{J}(q, z)
$$

is sequentially lower semicontinuous.

Indeed let the sequence $\left(z^{k}\right) \subset \mathcal{U}_{a d}$ converge in $\left(L_{l o c}^{1}[0,1]\right)^{3}$ to $z_{0}$. We have that $z_{0} \in \mathcal{U}_{a d}$ using property $(\mathrm{P} 2)$. We have also that $q\left(z^{k}\right) \rightarrow q\left(z_{0}\right)$ (Godlewski and Raviart [22]). It follows from property P4) that

$$
\underline{\lim }_{k \rightarrow \infty} \mathbf{J}\left(q\left(z^{k}\right), z^{k}\right) \geq \mathbf{J}\left(q\left(z_{0}\right), z_{0}\right)
$$

which establishes the lower semicontinuity of the operator defined in (18).

Finally let $\left(z^{j}\right)$ be a minimizing sequence for the optimal control problem (OPT). Using compactness of $\mathcal{U}_{a d}$ there exists a subsequence which converges to $\hat{\mathbf{z}} \in \mathcal{U}_{a d}$. We have proven that the operator (18) is sequentially lower semicontinuous which implies that $\hat{\mathbf{z}}$ is a solution for the optimal control problem (OPT) and this concludes the proof of existence of solutions for (OPT). 


\section{Minimization algorithms}

In order to minimize the above described cost functional we employed both nonsmooth and smooth optimization algorithms. A description of specific algorithms from each class implemented in this research is provided in the next tw subsections.

\subsection{Nonsmooth Optimization}

Since the gradient of a nonsmooth function $f$ exists only almost anywhere we have to replace the gradient by the generalized gradient

$$
\begin{array}{r}
\partial f(x)=\operatorname{conv}\left\{g \mid \text { there exists a sequence }\left(x_{i}\right)_{i \in \mathbf{N}} \text { such that } \lim _{i \rightarrow \infty} x_{i}=x,\right. \\
\left.f \text { differentiable at } x_{i}, i \in \mathbf{N}, \text { and } \lim _{i \rightarrow \infty} \nabla f\left(x_{i}\right)=g\right\}
\end{array}
$$

where "conv" is the notation for the convex hull.

We assume that we can compute the value of the function and an arbitrary subgradient $g \in \partial f(x)$ (i.e. one element of the generalized gradient) at any point in the domain.

The most efficient globally convergent algorithms for nonconvex nonsmooth

optimization are based on versions of the bundle methods (e.g. Lemarechal [38], Bonnans et al. [7], Schramm and Zowe [58], Makela and Neittaanmaki [47]). We employed a hybrid method (described in Vlcek and Luksan [68] and Luksan and Vlcek [46]) which combines the characteristics of the variable metric method and the bundle method.

The algorithm generates a sequence of basic points $\left(x_{k}\right)_{k \in \mathbf{N}}$ and a sequence of 
trial points $\left(y_{k}\right)_{k \in \mathbf{N}}$ satisfying

$$
x_{k+1}=x_{k}+t_{L}^{k} d_{k}, \quad y_{k+1}=x_{k}+t_{R}^{k} d_{k}
$$

with $y_{1}=x_{1}$, where $t_{R}^{k} \in\left(0, t_{\max }\right], t_{L}^{k} \in\left[0, t_{R}^{k}\right]$ are appropriately chosen stepsizes, $d_{k}=-H_{k} \tilde{g_{k}}$ is a direction vector, $\tilde{g_{k}}$ is an aggregate subgradient and the matrix $H_{k}$ accumulates information about the previous subgradients and represents an approximation of the inverse Hessian matrix if the function $f$ is smooth.

If the descent condition $f\left(y_{k+1}\right) \leq f\left(x_{k}\right)-c_{L} t_{R}^{k} w_{k}$ is satisfied with suitable $t_{R}^{k}$, where $c_{L} \in(0,0.5)$ is fixed and $-w_{k}<0$ represents the desirable amount of descent, then $x_{k+1}=y_{k+1}$ (descent step). Otherwise a null step is taken which keeps the basic points unchanged but accumulates information about the minimized function.

The aggregation is defined in the following way: denoting by $m$ the lowest index $j$ satisfying $x_{j}=x_{k}$ (index of the iteration after last descent step) and having the basic subgradient $g_{m} \in \partial f\left(x_{k}\right)$, the trial subgradient $g_{k+1} \in \partial f\left(y_{k+1}\right)$, and the current aggregate subgradient $\tilde{g}_{k}$, we define $\tilde{g}_{k+1}$ as a convex combination of these subgradients:

$$
\tilde{g}_{k+1}=\lambda_{k, 1} g_{m}+\lambda_{k, 2} g_{k+1}+\lambda_{k, 3} \tilde{g_{k}}
$$

where the multipliers $\lambda_{k}$ can be determined easily by minimizing a simple quadratic function which depends on these three subgradients and two subgradient locality measures (this approach replaces the solution of a rather complicated quadratic programming problem which appears in the standard bundle method Lemarechal [38]). 
The matrices $H_{k}$ are generated using a symmetric rank-one update after the null steps (to preserve the property of being bounded and other characteristics required for the global convergence) or the standard BFGS update after the descent steps (for both types of updates see Fletcher [16]).

\subsection{L-BFGS Unconstrained optimization algorithm}

We also tested the L-BFGS method (Nocedal [54], Liu and Nocedal [43], Nocedal and Wright [55]) which performs the unconstrained minimization of a smooth nonlinear function for which the gradient is available. L-BFGS is a limited memory method based on the well-known BFGS (Broyden-FletcherGoldfarb-Shanno) algorithm.

The main idea of this method is to use curvature information from only the mostrecent iterations to construct the Hessian approximation. Instead of storing fully dense $n \times n$ approximations it saves just a few vectors of length $n$ that represent the approoximations implicitly.

Each step of the original BFGS method has the form

$$
x_{k+1}=x_{k}-\alpha_{k} H_{k} \nabla J_{k}, \quad k=0,1,2, \ldots
$$

where $\alpha_{k}$ is the step length and $J$ is the cost functional. $H_{k}$ is updated at each ietration by means of the formula

$$
H_{k+1}=V_{k}^{T} H_{k} V_{k}+\beta_{k} s_{k} s_{k}^{T}
$$

where

$$
\beta_{k}=\frac{1}{y_{k}^{T} s_{k}} \quad V_{K}=I-\beta_{k} y_{k} s_{k}^{T}
$$


and

$$
s_{k}=x_{k+1}-x_{k} \quad y_{k}=\nabla J_{k+1}-\nabla J_{k}
$$

We say that the matrix $H_{k+1}$ is obtained by updating $H_{k}$ using the pair $\left(s_{k}, y_{k}\right)$. For L-BFGS a modified version of $H_{k}$ is stored implicitly, by using a certain number (say $m$ ) of the vector pairs $\left(s_{l}, y_{l}\right)$ that are used in the formulae (19)$(21)$

The product $H_{k} \nabla J_{k}$ can be obtained by performing a sequence of inner products and vector summations involving $\nabla J_{k}$ and the pairs $\left(s_{l}, y_{l}\right)$. After the new iterate is computed, the oldest vector pair in the set of pairs $\left(s_{l}, y_{l}\right)$ is deleted and replaced by the new pair $\left(s_{k}, y_{k}\right)$ obtained from the current step (21). In this way the set of vector pairs includes curvature information from the $m$ most recent iterations (usually $3 \leq m \leq 10$ ).

For numerical experiments using the L-BFGS method the reader is referred to Zou et al. [73].

We would like to conclude this section discussing our preference for L-BFGS over other smooth minimization algorithms. One may argue that for our case the number of control parameters may not justify the selection of a limited memory method. We consider that our approach (using the adjoint method for the gradient computation) may be easily and succesfully implemented for optimal control problems with a much greater number of control variables. In that case improvements in the efficiency of the numerical optimization will be determined not only by choosing the adjoint method over other methods for the gradient calculation but also by selecting a limited memory minimization algorithm. 


\section{Detection of discontinuities in data}

In the setting of smooth optimization one may consider that, by eliminating some discontinuities from the computation of the cost functional and its gradient (or subgradient) one may obtain a function which is smoother (i.e. more suitable for smooth optimization). Several approaches can be found in literature for the detection of discontinuities.

The discontinuity locking system (DLS) is employed for differential-algebraic

equations (DAE) by Birta and Oren [6], Park and Barton [57], Mao and Petzold [48], to cite but a few. The idea for this approach (DLS) is to lock the function evaluator for the initial-value problem solver so that the equations evaluated are fixed while an integration step is being taken, thus presenting a smooth vector field to the solver.

The approach we employ here is a modified application of a discrete regularization method proposed by Lee and Pavlidis [36] which is presented below.

Let $\left(x_{i}, y_{i}\right)_{i=0 \cdots n}$ be the set of data points with $x_{i}<x_{i+1}$. We want to find the $n+1$ quantities $z_{i}$ that minimize a combination of the discrete curvature and the discrete difference between observation and desired data:

$$
\sum_{i=0}^{n} \alpha_{i}\left(\frac{z_{i+1}-z_{i}}{x_{i+1}-x_{i}}-\frac{z_{i}-z_{i-1}}{x_{i}-x_{i-1}}\right)^{2}+\beta \sum_{i=0}^{n}\left(z_{i}-y_{i}\right)^{2}
$$

with $\alpha_{0}=\alpha_{n}=0$ and $\alpha_{i}=1$ for $i=1, \cdots, n-1$.

Differentiating (22) with respect to $z_{k}$ and setting the derivatives to zero yields a system of $n+1$ equations with $n+1$ equations, namely:

$$
P_{k 0} z_{k+2}-\left(P_{k 1}+P_{k 0}+P_{k-1,0}\right) z_{k+1}+\left(P_{k 1}+P_{k 2}+2 P_{k-1,0}+\beta\right) z_{k}
$$




$$
-\left(P_{k 2}+P_{k 3}+P_{k-1,0}\right) z_{k-1}+P_{k 3} z_{k-2}=\beta y_{k}
$$

where

$$
\begin{aligned}
P_{k 0} & =\frac{\alpha_{k+1}}{\left(x_{k+2}-x_{k+1}\right)\left(x_{k+1}-x_{k}\right)} \\
P_{k 1} & =\frac{\alpha_{k+1}+\alpha_{k}}{\left(x_{k+1}-x_{k}\right)^{2}} \\
P_{k 2} & =\frac{\alpha_{k-1}+\alpha_{k}}{\left(x_{k}-x_{k-1}\right)^{2}} \\
P_{k 3} & =\frac{\alpha_{k-1}}{\left(x_{k}-x_{k-1}\right)\left(x_{k-1}-x_{k-2}\right)}
\end{aligned}
$$

and $z_{-2}=z_{-1}=z_{n+1}=z_{n+2}=0$.

The parameter $\beta$ is chosen such that it satisfies:

$$
\beta \gg \frac{1}{\min _{k}\left(x_{k+1}-x_{k}\right)^{2}}
$$

which implies diagonal dominance for the system of equations (23). To find discontinuities in the function or for its derivative we look at zero crossings of the error between the observation and the desired data

$$
z_{i}-y_{i}
$$

and at zero crossings of the approximate curvature

$$
\frac{z_{i+1}-z_{i}}{x_{i+1}-x_{i}}-\frac{z_{i}-z_{i-1}}{x_{i}-x_{i-1}}
$$

Slope discontinuities are characterized by successive zero crossings of type (23) and function discontinuities are characterized by zero crossings of type (23) and (24).

For our problem we are interested in eliminating only the points which are associated with the shocks (this is a trade-off between obtaining a smoother 
function and preserving as much as possible the discontinuous character of the problem for a given time interval). If one would like to single out a region of the solution (among the five regions described in section 2) which has the greatest influence during numerical optimization one would select the points where the shock occurs.

The detection of shock points was performed by considering only points with approximate curvatures above a certain threshold value. This approach was suggested to us by the fact that the curvature for the analytical solution is very steep in the shock region. 


\section{Numerical results}

The main goal for this research was to control the location of the discontinuities by matching the numerical flow to a desired flow. The cost functional was taken to be the (weighted) difference between the numerical and the desired solution. We chose the desired flow to be the exact solution of the analytical problem.

We wanted our research to include many characteristics of the class of the optimal control problems with discontinuities. Since one of the most important features for this class of problems is the character of nonsmoothness for the cost functional, one may argue that successful minimization of a very nonsmooth cost functional in 1-D with a small number of control variables will determine a greater level of confidence for the extension of this approach to problems in higher dimensions and with a much larger number of control variables. We consider that our choice of observations contributed to a greater nonsmoothness of the cost functional and, as a consequence, to improve the generality of the conclusions derived from this research.

For many problems (including ours) the problem of finding a "matching" flow at a given time is equivalent to the problem of finding the corresponding vector of initial conditions ( the initial conditions serve as the control variables in the optimal control setting).

For the practical applications of this approach (namely controlling the location of the discontinuities) it is more important to consider the impact on the flow parameters due to the change of shock location rather than the explicit description of the new discontinuity location. For this reason we concentrated our research efforts on matching the flow to a desired flow rather than intro- 
ducing the explicit shock location as a variable in the optimal control setup (as performed by Cliff et al. [11] for duct flow with quasi 1-D Euler equations).

As presented in Appendix A our method uses the discrete (forward) model to obtain the tangent linear model and then the adjoint model which provides the gradient or a subgradient of the cost functional to the smooth (nonsmooth) minimizer. If the location of the discontinuities were to be introduced as an explicit variable then the original code will have to be modified to accommodate the new requirements, a change requiring complex adjustments.

For each of the two numerical models chosen for study in this article (artificial viscosity model AVM or high-resolution model HRM) we employed unconstrained optimization methods (L-BFGS algorithm for smooth optimization and PVAR algorithm for nonsmooth optimization) described in section 5 .

The control variables were chosen to be the initial parameters to the left and to the right of the membrane: pressure $p_{L}, p_{R}$ and density $\rho_{L}, \rho_{R}$. The desired observations were obtained as exact solutions of the shock-tube problem at times $t=0.15$ or $t=0.24$ starting with prescribed initial conditions (which will be referred to as the first set of parameters

$$
\left(\rho_{L}=1.1, p_{L}=1.1, \rho_{R}=0.2, p_{R}=0.2\right)
$$

and respectively the second set of parameters

$$
\left(\rho_{L}=1.2, p_{L}=1.2, \rho_{R}=0.3, p_{R}=0.3\right)
$$

The initial guess for both sets of desired observations and for both optimization methods was taken to be

$$
\left(\rho_{L}=1.0, p_{L}=1.0, \rho_{R}=0.1, p_{R}=0.1\right)
$$


characteristic for Sod shock-tube problem $([60])$. The initial values for velocities to the left and to the right of the membrane were taken to be zero. Figures (5)-(8) show on the same graph the observations and the numerical solution obtained at the same time $t_{\text {final }}$ for the AVM or HRM model with the initial guess for the control variables serving as the initial conditions.

Comparing the initial guess with the desired values for the control parameters one may argue that the distance between them is very small which suggests a trivial optimization problem. This remark would be valid for the case of smooth functions fow which a small change in the initial conditions implies a rather small change in the output values. However, a careful study of the cost functionals described below shows that the small perturbation in the initial conditions (of order $10^{-1}$ ) determines a difference in the outputs of order $10^{2}$ or $10^{3}$.

As discussed in the section 4 (existence of solutions for the optimal control problem) we consider two expressions for the cost functional, with either observation at the end of the assimilation window or distributed observations in time.

If the observation was obtained at the end of the time window $\left(t=T_{W}\right)$ the following discrete form of the cost functional was considered:

$$
\begin{aligned}
J(\mathbf{U}(\cdot, 0), \mathbf{P}(\cdot, 0), \rho(\cdot, 0))= & \sum_{i=1}^{N \text { points }}\left(W_{\mathbf{U}}(i) \times\left(\mathbf{U}^{\text {num }}(i)-\mathbf{U}^{\text {obs }}(i)\right)^{2}\right. \\
& +W_{\mathbf{P}}(i) \times\left(\mathbf{P}^{\text {num }}(i)-\mathbf{P}^{\text {obs }}(i)\right)^{2} \\
& \left.+W_{\rho} \times\left(\rho^{\text {num }}(i)-\rho^{\text {obs }}(i)\right)^{2}\right)
\end{aligned}
$$


where

$$
\mathbf{U}(x, 0)=\left\{\begin{array}{ll}
0.0, x<0.5 \\
0.0, x>0.5
\end{array} \quad \mathbf{P}(x, 0)=\left\{\begin{array}{ll}
p_{L}, x<0.5 \\
p_{R}, x>0.5
\end{array} \quad \rho(x, 0)=\left\{\begin{array}{l}
\rho_{L}, x<0.5 \\
\rho_{R}, x>0.5
\end{array}\right.\right.\right.
$$

with $\left(\rho_{L}, p_{L}, \rho_{R}, p_{R}\right)$ the control variables described above, Npoints is the number of points for space discretization, $W_{\mathbf{U}}, W_{\mathbf{P}}, W_{\rho}$ are the weights attached to points, $\mathbf{U}^{\text {num }}, \mathbf{P}^{\text {num }}, \rho^{\text {num }}$ are the fields of velocity, pressure and density at time $t_{\text {final }}$ while $\mathbf{U}^{o b s}, \mathbf{P}^{o b s}, \rho^{o b s}$ are the observations for velocity, pressure and density.

For distributed observations the discrete form of the cost functional is:

$$
\begin{gathered}
J(\mathbf{U}(\cdot, 0), \mathbf{P}(\cdot, 0), \rho(\cdot, 0))=\sum_{j=1}^{\text {Nobs }} \sum_{i=1}^{\text {Npoints }}\left(W_{\mathbf{U}}(i) \times\left(\mathbf{U}_{(j)}^{\text {num }}(i)-\mathbf{U}_{(j)}^{\text {obs }}(i)\right)^{2}\right. \\
\quad+W_{\mathbf{P}}(i) \times\left(\mathbf{P}_{(j)}^{\text {num }}(i)-\mathbf{P}_{(j)}^{\text {obs }}(i)\right)^{2} \\
\left.\quad+W_{\rho} \times\left(\rho_{(j)}^{\text {num }}(i)-\rho_{(j)}^{\text {obs }}(i)\right)^{2}\right)
\end{gathered}
$$

where in addition to the notations described above for the previous cost functional we have Nobs the number of instances during the assimilation window for which we consider the observations, $\mathbf{U}_{(j)}^{\text {num }}, \mathbf{P}_{(j)}^{\text {num }}, \rho_{(j)}^{\text {num }}$ are the fields of velocity, pressure and density at time $t_{(j)},(1 \leq j \leq N o b s)$ while $\mathbf{U}_{(j)}^{o b s}, \mathbf{P}_{(j)}^{o b s}, \rho_{(j)}^{o b s}$ are the observations for velocity, pressure and density at the same observation times $t_{(j)}$.

The control variables employed for this research were the initial values for presure and density. The desired value for the initial velocity is 0.0 (both to the left and to the right of the membrane) and for this reason we did not consider the initial value of the velocity among the control variables. Another reason for selecting the initial values of the velocity to be zero is related to the 
physical aspects of the shock-tube problem. If the initial values for the velocity are considered control variables then during the minimization their updates may have values which are different than zero (either positive or negative values). The result may be either not physical or the corresponding variables may develop bifurcation points for which special additional issues should be addressed (Cacuci [8]).

The numerical results for both models (AVM and HRM) using either one of the optimization methods (L-BFGS and PVAR) are presented in Figures (9)-(27) as well as entries in the Tables (1)-(8). Figures (9)-(19) present the evolution of the cost functional and of the norm of the gradient (or subgradient) for the cost functional. The norms employed are the discrete versions of the norms in $L^{2}$ (gradient) and in $L^{\infty}$ (subgradient). Figures (20)-(27) show the numerical solution obtained as a result of the optimal control and the values of the optimized control parameters are presented in Tables (1)-(8).

We considered two time windows for the optimal control problem: $T_{W}=0.15$ or $T_{W}=0.24$ (in nondimensional units) which were chosen such that at the end of the time window the flow exhibits all five regions of discontinuities discussed in Section 2. If one increases the time window from $T_{W}=0.24$ to time $=0.3$ one can see from the Figure 29 that several characteristics of the discontinuities have already disappeared. For these reasons our choices of time windows are representative of the shocked flow problem.

For the HRM model the optimized values of the control parameters are in excellent agreement with the parameters' desired values for both sets of observations and for both assimilation windows if the nonsmooth optimization package PVAR was employed. Figures (9) and (10) show a decrease of more 
than 2 orders of magnitude for the cost functional. The optimal values of the control parameters

$$
\left(\rho_{L}, p_{L}, \rho_{R}, p_{R}\right)
$$

obtained as a result of nonsmooth optimization (the column PVAR in Tables (1)-(4) display a very good agreement with the desired parameters. See also Figures (20)-(21) for a comparison between the numerical optimized solution and the observations.

The optimization has essentially converged in about 25 iterations (for the first set of observations) and in about 60 iterations (for the second set of observations) for the same prescribed convergence criterion.

For the model HRM we also employed a cost functional with distributed (in time and space) observations for the larger time window $T_{W}=0.24$. The optimized values of the control parameters obtained as a result of the nonsmooth minimization PVAR are shown as entries in the column $\mathbf{P V A R}($ d.c.) (e.g. distributed controls) in Tables (2)-(4).

It can be seen from the Tables that a very good agreement was obtained with the desired values of the parameters. The corresponding Figures show that the flow obtained with the optimized control parameters as initial conditions matches very closely the observations and, in the same time, that the new location of the discontinuities matches the desired location.

One may argue that the results obtained are slightly better for distributed observations if one considers the third or fourth digit after the decimal point, which proves that we have already obtained excellent optimized results (almost identical to the desired values of the parameters) for a cost functional 
computed using only final time observations. The graphs (9) and (10) show the evolution of the distributed observations cost functional and the norm of its subgradient versus the number of minimization iterations.

For the same model (HRM) the L-BFGS minimization was employed for both time windows. This method converged only for the shorter time window $\left(T_{W}=0.15\right)$ for which we obtained similar results to PVAR as seen in the columns LBFGS and PVAR in Tables (1)-(3). For the larger time window the L-BFGS minimization did not perform successfully. It failed completely for the first set of observations (although the failure was not due to the minimizer as much as a consequence of the fact that some optimization updates did not qualify as correct physical solutions for the forward model, an aspect which is discussed below). For the second set of observations the optimized results did not converge to the desired parameters (Table (4)). This failure suggests that the the computation of the gradient by the adjoint method is more impacted by the presence of discontinuities when a larger time window is considered.

For many numerical models which smooth the discontinuities we may consider that the cost functional is "numerically" smoother. For this reason we also tested unconstrained smooth minimization methods using the gradient derived from the adjoint model obtained using a forward code with an artificial viscosity term AVM). The evolution of the cost functional and that of the norm of its gradient for the AVM model are displayed in Figures (13)-(19) while the optimized results derived from solving the optimal control problem are shown in Tables (5)-(8).

For the AVM model the nonsmooth optimization method PVAR performed successfully for both sets of above mentioned observations as was the case for 
the HRM model. The main difference was observed in the memory and CPU time requirements). The HRM model employs much fewer points and time steps compared to the AVM model: for comparable accuracy the number of mesh points for HRM model was 200 while it was 500 for AVM; respectively for the time window $T_{W}=0.24$ the HRM forward model required 192 time steps while the AVM forward model required 1000 timesteps).

The cost functional of the AVM model decreased by 3 orders of magnitude (attaining a similar order of magnitude as that obtained for the HRM model but starting at a larger value of the cost functional due to the higher number of mesh points. The number of iterations required for convergence of the AVM model was almost the same as that for the HRM model, the only improvement being observed for the time window $T_{W}=0.15$ and for the second set of observations (in fact for the time window $T_{W}=0.24$ the number of iterations increased slightly compared to HRM model). A sharper decrease is observed for the norm of the gradient due to the fact that the discontinuities in the forward AVM model are smeared causing a smoother input of the adjoint model.

The L-BFGS unconstrained minimization was successful in minimizing the cost functional for both sets of observations only for the time window $T_{W}=$ 0.15. The evolution of the cost functional and the norm of its gradient are shown in Figure (19). For the time window $T_{W}=0.24$ this method was successful only for the second set of observations as seen in Figure (18) and Table (8) respectively. For the first set of observations the results obtained using the algorithm L-BFGS did not converge to the desired parameters (Table (6)), but to an output which was consequently utilized as an initial guess for the PVAR method. The evolution of the cost functional in this case is shown 
in Figure (30). If we compare it with Figure (13) we notice that, while the total number of iterations increased, the number of iterations which were effectively used for achieving the minimization of the cost functional decreased from 25 iterations to 12 iterations (thereafter the decrease of the cost functional "stalls").

We would like to describe in more detail the "failure" of the L-BFGS method for our problem. For some cases (e.g. for HRM model using the first set of observations and time window $\left.T_{W}=0.24\right)$ the minimization per se performed successfully (i.e. a new step size was obtained and the vector of control variables was updated) but the updated vector of control variables did not qualify as a solution from the physical point of view although the requirements for an update from the optimization point of view (i.e. decrease of the cost functional) were satisfied. A typical example of optimization update encountered during the "failed" L-BFGS minimization may serve for better understanding of possible causes for failure in our case: the control parameters were updated to new values for which the forward model could not compute the numerical flow and, as a consequence, the cost functional and its gradient were not available for the next minimization iteration.

We applied scaling for the gradient of the cost functional in the cases when L-BFGS unconstrained optimization failed. We did not consider scaling for the cases with convergence to the desired parameters but we may assume that by employing scaling in thosecases one would achieve convergence in fewer minimization steps (although very little improvement will be seen for the optimized control parameters since they are in excellent agreement with the desired parameters even without scaling). 
The scaling was chosen such that all components of the gradient have numerical values of order one. An example of gradient scaling characteristic for all the cases considered is presented here: the original gradient was the vector

$$
(50.95,66236.07,37.82,-66323.43)
$$

and after scaling the new vector gradient became

$$
(5.09,6.62,3.78,-6.63)
$$

The results of L-BFGS optimization with scaled gradient are presented in the column L-BFGS scaled of the Tables (2), (4) and (6). We notice that L-BFGS optimization algorithm with scaling did not fail as for the case of L-BFGS optimization algorithm without scaling (the column L-BFGS in the same Tables). But the method did not converge to the desired values of control parameters.

Since the main reason for the poor performance of L-BFGS may be attributed to the presence of discontinuities we tested a method whereby we selectively applied weights to the points of discontinuities. These points were found by employing a discontinuity detection method described in Section 6.

The solution for shock-tube problem comprises of several distinct regions including contact discontinuities, rarefaction waves and shocks. The choice of the points where the weights were applied was based on the trade-off between the desire for a smoother function and the requirement of preserving as much as possible of the properties for the original problem. For this reason we decided upon assigning the weights only to the points where the shock occurs. Figure (28) shows the "shock" points selected from the entire number of space points. 
Different weights $($ weight $=0.0$, weight $=1.0$, weight $=25.0)$ were considered in the computation the cost functional and its gradient (weight $=0.0$ means removal of these points from the cost functional and its gradient computation, for weight $=1.0$ all the mesh points are considered to have the same influence respectively for weight $=25.0$ the influence of the shock is dominant).

These weights were applied to the cost functional and its gradient only if the AVM model was employed for the forward model. At each minimization iteration the method of discontinuity detection was reapplied and the corresponding shock points were found (the update for the control vector gives new initial conditions e.g. the shock changes its location in the forward model and consequently in the adjoint model).

For both sets of observations and for both methods of optimization the weighted minimization with weight $=25.0$ failed for time windows $T_{W}=0.15$ and $T_{W}=0.24$. The non smoothness of the cost functional was accentuated in such a way that even the method of nonsmooth optimization (which previously performed in a very robust way) had severe difficulties to perform adequately and did not converge.

In the case of weight $=0.0$ a successful minimization was obtained for both sets of observations and for both time windows as seen in the column L$\operatorname{BFGS}(w=0)$ of Tables (5)-(8) (even for the case of time window $T_{W}=0.24$ and first set of observations, for which L-BFGS did not converge previously). The evolution of the cost functional and the behavior of the norm of its gradient are presented in Figures (15) and (16). Comparing them with the evolution of the same quantities described in Figures (18) and (19) we observe that the number of minimization iterations decreased by a factor of two. When we com- 
pared the optimized values of the control parameters in Tables (5)-(8) for the case weight $=0.0$ with the same values for the case "no weight considered" (which, in fact, is equivalent to assignment of a unit weight) we obtained very satisfactorily results for both cases.

We tested the weighted cost functional with weight $=0.0$ in the case of nonsmooth optimization for the time window $T_{W}=0.15$ and for both sets of observations. Although a small reduction in the number of iterations was obtained we did not consider that the magnitude of this reduction justifies this approach for the larger time window (in addition, by eliminating the shock points from the cost functional computation the method of nonsmooth minimization may disregard some nonsmooth characteristics of the problem which may prove very useful from the physical point of view). 


\section{Summary and Conclusions}

We solved an optimal control problem for a flow which includes several types of discontinuities (namely we carried out a flow matching for a 1-D Riemann problem for Euler equations: shock-tube problem). The optimal controls considered were the initial conditions at the left and at the right of the membrane for pressure and density. Existence results were proven for the solution of the described optimal control problem.

Here flow matching was equivalent to relocation of discontinuities to a desired location. Since in all practical control applications discontinuities are captured using either high-resolution models or models which smooth the solution we employed here two numerical models representative of both approaches.

The cost functional was taken to be the (weighted) difference between the numerical and the desired solution. The observations were taken either at the end of the time window or we had observations distributed in space and time within the assimilation time window.

For each forward code its corresponding adjoint model was then employed for computing the gradient (or a subgradient) of the cost functional required for carrying out the minimization of the cost functional (either nonsmooth or smooth algorithms for optimization). The two assimilation windows for minimization were chosen such that the flow with discontinuities retained all its characteristics at the end of each time window. The flow changes some of these characteristics if we were to use a slightly larger time window.

The method of nonsmooth optimization (PVAR) employed for minimizing 
the cost functional was found to be very robust. For each of the different sets of observations employed we obtained optimized values of the control parameters which were in very good agreement with the desired results. The method of smooth optimization (LBFGS) provided good results only in the case of the shorter time window and failed for the longer time window.

For the artificial viscosity model we tested a method of weights (which were attached to the points were the shock occurs) for the cost functional and its gradient. The shock points were identified using a method of discontinuities detection described in Section 5. When the weights were chosen to be 0.0 (i.e. removal of the shock region) the number of iterations required for attaining a prescribed convergence when using the smooth minimization LBFGS was reduced by a factor of two.

A very useful characteristic of the methodology for optimal control for discontinuous flow presented in this article is the ease with which it can be implemented in applications where the forward model is already discretized.

Although this research was performed for a 1-D space and time problem, we consider that one can make a very good argument for the extension of the present methodology to models in higher space dimensions (due to the fact that many types of discontinuities encountered in higher dimensions appear also in the solution of this 1-D optimal control problem). Extending this approach to optimal control problems with discontinuities in 2-D or 3-D makes the adjoint method even more appealing computationally, due to the much larger number of control parameters involved.

A very important topic which will be addressed in future research is related to the issue of noisy observations. One may expect in this case that the cost 
functional will have new components which will account for the effect of the noise.

We consider our research is a only small step towards the complete solution of optimal control of problems with discontinuities. Although the number of research works discussing numerical aspects for optimal control problems with discontinuities is extremely small we are confident that, given the importance of this subject, many other researchers will dedicate their efforts to advancing the knowledge for this class of problems 


\section{Appendix :Computation of the gradient (a subgradient) of the cost functional using the adjoint method}

Assuming that the cost functional has the following form:

$$
\mathbf{J}[X, \Lambda]=\frac{1}{2} \sum_{k=0}^{R}\left[\mathbf{X}\left(t_{k}\right)-\mathbf{X}^{o b s}\left(t_{k}\right)\right]^{T} \mathbf{W}\left(t_{k}\right)\left[\mathbf{X}\left(t_{k}\right)-\mathbf{X}^{o b s}\left(t_{k}\right)\right]
$$

with $\mathbf{W}\left(t_{k}\right)$ being a diagonal weighting matrix, $t_{0} \leq t_{k} \leq t_{R}$, $\left[t_{0}, t_{R}\right]$ the minimization window, $R$ the number of time steps in the minimization window and $\Lambda$ the vector of control parameters respectively, the gradient (a subgradient) of the cost functional with respect to the control parameters: $\left(\nabla_{\Lambda} \mathbf{J}[\Lambda]\right)^{T}$ is computed using the adjoint method.

The notions of gradient or subgradient are employed here in the numerical sense (the analytical Riemann problem has only subgradients due to the presence of discontinuities). As result of the adjoint method we obtained the numerical gradient in the case of smoothed model and respectively one numerical subgradient in the case of the high-resolution model.

We define the adjoint equations for the adjoint variables $\hat{\Lambda}^{(k)}$ :

$$
\hat{\boldsymbol{\Lambda}}^{(k)}\left(t_{0}\right)=\mathbf{Q}_{k}^{T} \hat{\mathbf{\Lambda}}^{(k)}\left(t_{k}\right), \text { for } k=1, \cdots, R
$$

If the adjoint variable $\hat{\boldsymbol{\Lambda}}^{(k)}(t)$ at time $t_{k}$ is initialized as:

$$
\hat{\Lambda}^{(k)}\left(t_{k}\right)=\mathbf{W}\left(t_{k}\right)\left[\mathbf{X}\left(t_{k}\right)-\mathbf{X}^{\text {obs }}\left(t_{k}\right)\right]
$$


then the gradient of the cost function with respect to the control parameters can be obtained as in Navon et al. [53]:

$$
\nabla_{\boldsymbol{\Lambda}} \mathbf{J}[X]=\sum_{k=0}^{R} \hat{\boldsymbol{\Lambda}}^{(k)}\left(t_{k}\right)
$$

If we linearize the nonlinear model about the current model solution we obtain the tangent linear model (TLM). The transpose of the TLM is the adjoint model.

If we view the tangent linear model as the result of the multiplication of a number of operator matrices: $\mathbf{A}_{1} \mathbf{A}_{2} \cdots \mathbf{A}_{M}$ where each matrix $A_{i}, i=1, \cdots, M$ represents either a subroutine or a single DO-loop, then the adjoint model can be viewed as being a product of adjoint subproblems: $\mathbf{A}_{M}^{T} \mathbf{A}_{M-1}^{T} \cdots \mathbf{A}_{1}^{T}$.

The correctness of the adjoint of each operator was checked using the following identity:

$$
(\mathbf{A Q})^{T}(\mathbf{A Q})=\mathbf{Q}^{T}\left(\mathbf{A}^{T}(\mathbf{A} \mathbf{Q})\right)
$$

where $\mathbf{Q}$ represents the input of the original code and $\mathbf{A}$ can be either a single DO loop or a subroutine. All subroutines of the adjoint model were subjected to this test.

The accuracy of the gradients calculated by the adjoint method should be at the level of machine precision. Errors could result due to coding mistakes, round-off errors or the presence of non differentiable functions.

For a smooth cost functional the correctness of the adjoint model is checked by examining the first-order approximations to a nonlinear perturbation of 
the cost functional (see Navon et al. [53]):

$$
\boldsymbol{\Phi}(\eta)=\frac{\mathbf{J}(\mathbf{X}+\eta \mathbf{h}))-\mathbf{J}(\mathbf{X})}{\eta \mathbf{h}^{\mathbf{T}} \nabla \mathbf{J}(\mathbf{X})}
$$

where $\|\mathbf{h}\|=1, \eta$ scalar and $\nabla \mathbf{J}(\mathbf{X})$ is the gradient of the cost functional $\mathbf{J}(\mathbf{X})$ with respect to $\mathbf{X}$ computed using the adjoint code.

For a differentiable cost functional the gradient derived from the adjoint model can be assumed to be completely accurate (up to the machine error) when $\lim _{\eta \rightarrow 0}|\Phi(\eta)|=1$. A validity region of the gradient test is normally obtained for $10^{-3} \geq \eta \geq \epsilon$ (where $\epsilon$ is the machine accuracy). For $\eta>10^{-3}$ we have truncation error and for $\eta$ near the machine accuracy roundoff errors prevail.

In our case the exact observations employed for the cost functional contain discontinuities which determine the nonsmooth character of the cost functional. We considered the gradient obtained from the adjoint model to be sufficiently accurate if the following tolerances were satisfied

$$
\lim _{\eta \rightarrow 0}|\boldsymbol{\Phi}(\eta)|=\delta
$$

for $10^{-3} \geq \eta \geq 10^{-10}$

where $\delta$ is a constant number which depends on the problem parameters. The results of the gradient check test are displayed in Figures (1) and (2).

From these graphs we can notice that the gradient ratio tends to a constant number in all cases which decreases slightly as we increase the time window. 


\section{Acknowledgments}

The authors acknowledge the support from the School of Computational Science and Information Technology, Florida State University. They would like to thank L. Luksan, R. Leveque, T. Cowan and G. Wahba for their insightful comments. The second author acknowledge the support from the NSF grant number ATM-9731472 managed by Dr. Linda Peng whom he would like to thank for her support.

\section{References}


[1] W.K. Anderson and A. Venkatakrishnan, Aerodynamic design optimization on unstructured grids with a continuous adjoint formulation, Comput. \& Fluids 28, 443(1999).

[2] E. Arian and M.D. Salas, Admitting the inadmissible: adjoint formulation for incomplete cost functionals in aerodynamic optimization, ICASE Report 97-69, 1997.

[3] P.I. Barton, J.R. Banga and S. Galan, Optimization of hybrid discrete/continuous dynamic systems, Comput. Chem. Eng. 24, 2171(2000).

[4] T. Bein, H. Hanselka and E. Breitbach, An adaptive spoiler to control the transonic shock, Smart Mater. Struct. 9(2), 141(2000).

[5] J. Birkmeyer, H. Rosemann and E. Stanewsky, Shock control on a swept wing, Aerosp. Sci. and Technol. 4(3), 147(2000).

[6] L.G. Birta and T.I. Oren, A robust procedure for discontinuity handling in continuous system simulation, T. Soc. Comput. Simul. 2(3), 189(1985).

[7] J.F. Bonnans, J. Gilbert, C. Lemarechal and C. Sagastizabal, Optimisation numerique: aspects theoriques et pratiques, (Springer-Verlag Paris, 1997).

[8] D.G. Cacuci, Global optimization and sensitivity analysis, Nucl. Sci. Eng. 104, 78(1990).

[9] E.M. Cliff, M. Heinkenschloss and A.R. Shenoy, Adjoint-based methods in aerodynamic design optimization, In Computational methods for optimal design and control (Birkhauser, 1996), p.91.

[10] E.M. Cliff, M. Heinkenschloss and A.R. Shenoy, On the optimality system for a 1-D Euler flow problem, AIAA Paper 96-3993, 1996.

[11] E.M. Cliff, M. Heinkenschloss and A.R. Shenoy, An optimal control problem for flows with discontinuities J. Optimiz. Theory App. 94(2), 273(1997). 
[12] T.J. Cowan, Private communication 2001.

[13] A. Dadone, M. Valorani and B. Grossman, Optimization of 2D fluid design problems with nonsmooth or noisy objective function In Computational Fluid Dynamics '96 (Wiley and Sons, 1996), p.425.

[14] A. Dadone and B. Grossman, Progressive optimization of inverse fluid dynamic design problems, Comput. \& Fluids 29, 1(2000).

[15] A. Dadone and B. Grossman, Fast convergence of inviscid fluid dynamic design problems. In Proceedings of ECCOMAS 2000, Barcelona 11-14 September 2000 www.imamod.ru/jour/conf/ECCOMAS2000/pdf/148.pdf

[16] R. Fletcher, Practical methods of optimization, (Wiley and Sons, 1987).

[17] P.D. Frank and G.Y. Shubin, A comparison of optimization-based approaches for a model computational aerodynamics design problem, J. Comput. Phys. 98, 74(1992).

[18] M.B. Giles and N.A. Pierce, Adjoint equations in CFD: duality, boundary conditions and solution behaviour, AIAA Paper 97-1850, 1997.

[19] M.B. Giles and N.A. Pierce, Analytic adjoint solutions for the quasi-1D Euler equations, J. Fluid Mech. 426, 327(2001).

[20] M.B. Giles and N.A. Pierce, An introduction to the adjoint approach to design, Flow Turbul. Combust. 65(3-4), 393(2000).

[21] M.B. Giles and N.A. Pierce, On the properties of solutions of the adjoint Euler equations. In Numerical Methods for Fluid Dynamics VI (ICFD, Oxford, 1998).

[22] E.Godlewski and P.A. Raviart, Numerical approximation of hyperbolic systems of conservation laws, (Springer-Verlag, 1996).

[23] M. Gunzburger, Sensitivities, Adjoints and Flow Optimization, Int. J. Numer. Meth. Fl. 31, 53(1999). 
[24] M. Gunzburger, Sensitivities in computational methods for optimal flow control. In Computational methods for optimal design and control, (Birkhauser, 1996), p.197.

[25] A. Habbal, Direct approach to the minimization of the maximal stress over an arch structure, J. Optimiz. Theory App. 97(3), 551(1998).

[26] A. Habbal, Nonsmooth shape optimization applied to linear acoustics, SIAM J. Optimiz. 8(4), 989(1998).

[27] E. Hassold, Automatic differentiation applied to a nonsmooth optimization problem. In Numerical Methods in Engineering '96, (Wiley, 1996), p.835.

[28] C. Homescu, I.M. Navon and Z. Li, Suppression of vortex shedding for flow around a circular cylinder using optimal control, Int. J. Numer. Meth. Fl. 38, $43(2002)$.

[29] C. Homescu and I.M. Navon, Numerical and theoretical considerations for sensitivity calculation of discontinuous flow, Accepted at Syst. Control Lett. (2002).

[30] L. Huyse and M.R. Lewis, Aerodynamic shape optimization of two-dimensional airfoils under uncertain conditions, ICASE Report 2001-1, 2001.

[31] A. Iollo A and M.D. Salas, Optimum transonic airfoils based on Euler equations Comput. \& Fluids 28, 653(1999).

[32] A. Iollo, M.D. Salas and S. Ta'asan, Shape optimization governed by the Euler equations using an adjoint method ICASE Report 93-78, 1993.

[33] A. Iollo A and M.D. Salas, Contribution to the optimal shape design of twodimensional internal flows with embedded shocks J. Comput. Phys. 125(1), 124(1996). 
[34] F. James and M. Sepulveda, Convergence results for the flux identification in a scalar conservation law, SIAM J. Control Optim. 37(3), 869(1999).

[35] A. Jameson, A perspective on computational algorithms for aerodynamic analysis and design, Prog. Aerosp. Sci. 37, 197(2001).

[36] D. Lee and T. Pavlidis, One-dimensional regularization with discontinuities, IEEE Trans. on Pattern Anal. Mach. Intell.10(6), 822(1988).

[37] C. Lemarechal, Nondifferentiable optimization, Subgradient and $\epsilon$ subgradient methods, In Optimization and Operations Research, Lecture Notes in Economics and Mathematical Systems 117, (Springer-Verlag, 1976), p.191.

[38] C. Lemarechal, Nondifferentiable optimization. In Handbooks in Operations Research and Management Science vol.1 Optimization, (North Holland, 1989).

[39] D. Leonard and N.V. Long, Optimal control theory and static optimization in eonomics, (Cambridge Univ. Press, 1992).

[40] R. Leveque, Numerical methods for conservation laws, (Birkhauser, 1992).

[41] CLAWPACK: A Software Package for Conservation Laws and Hyperbolic Systems. http://www.amath.washington.edu/ rjl/clawpack

[42] H.W. Liepmann and A. Roshko, Elements of gas dynamics, (Wiley and Sons, 1957).

[43] D. Liu and J. Nocedal, On the limited memory BFGS method for large scale optimization Math. Program. B, 45, 503(1989).

[44] R. Lohner, K. Morgan, J. Peraire and M. Vahdati, Finite element flux-corrected transport (FEM-FCT) for the Euler and Navier-Stokes equations. Int. J. Numer. Meth. Fl. 1987; 7 :1093-1109 
[45] L. Luksan and J. Vlcek, NDA: Algorithms for nondifferentiable optimization, Institute of Computer Science, Academy of Sciences of the Czech Republic, ICS Report 797(2000).

[46] L. Luksan and J. Vlcek, Algorithm811: NDA: Algorithms for nondifferentiable optimization, ACM T. Math. Software,27(2), 193(200).

[47] M.M. Makela and P. Neittaanmaki, Nonsmooth Optimization, World Scientific, 1992.

[48] G. Mao and L.R. Petzold, Efficient integration over discontinuities for differential-algebraic systems, Comput. Math. Appl.43, 65(2002).

[49] T. Matsuzawa and M. Hafez, Optimum shape design using adjoint equations for compressible flows with shock waves, Computational Fluid Dynamics 7(3), 343(1998).

[50] T. Matsuzawa and M. Hafez, Treatment of shock waves in design optimization via adjoint equation approach, Computational Fluid Dynamics 7(4), 405(1998).

[51] B. Mohammadi and O. Pironneau, Applied Shape Optimization for Fluids, (Oxford Science Publications, 2001).

[52] R.P. Narducci, B. Grossman and R.T Haftka, Sensitivity algorithms for an inverse design problem involving a shock wave, Inverse Probl. Eng.2, 49(1995).

[53] I.M. Navon, X. Zou, J. Derber and J. Sela, Variational data assimilation with an adiabatic version of the NMC spectral model, Mon. Wea. Rev.120, 1433(1992).

[54] J. Nocedal, Updating Quasi-Newton matrices with limited storage, Math. Comput.151(35), 773(1980).

[55] J. Nocedal and S.J. Wright, Numerical Optimization, (Springer, 1999).

[56] A. Oyama, S. Obayashi and K. Nakahashi, Transonic wing optimization using genetic algorithm, AIAA Paper 97-1854, 1997. 
[57] T. Park and P.I. Barton, State event location in differential-algebraic models, ACM Trans. Model. Comput. Simul. 6(2), 137(1996).

[58] H. Schramm and J. Zowe, A version of the bundle idea for minimizing a nonsmooth function: conceptual idea, convergence analysis, numerical results, SIAM J. Optim.2(1), 121(1992).

[59] F. Shakib, T.J.R. Hughes and Z. Johan, A new finite formulation for computational fluid dynamics: X. The compressible Euler and Navier Stokes equation, Comput. Methods Appl. Mech. Eng. 89, 141(1991).

[60] G.A. Sod, A survey of finite-difference methods for systems of nonlinear conservation laws, J. Comput. Phys.27(1), 1(1978).

[61] E. Stanewsky, Adaptive wing and flow control technology, Prog. Aerosp. Sci.37, $583(2001)$.

[62] J.E. Tolsma and P.L. Barton, Hidden discontinuities and parametric sensitivity calculations, SIAM J. Sci. Comput.23(6), 1862(2002).

[63] M. Valorani and A. Dadone, Sensitivity derivatives for non-smooth or noisy objective functions in fluid design problems, In Numerical Methods for Fluid Dynamics, vol. 5, (Oxford Clarendon Press, 1995), p.605.

[64] S. Ulbrich, On the existence and approximation of solutions for the optimal control of nonlinear hyperbolic conservation laws, In Optimal control of partial differential equations (Chemnitz 1998) International Series of Numerical Mathematics 133, (Birkhauser, 1999), p.287.

[65] S. Ulbrich, A sensitivity and adjoint calculus for discontinuous solutions of hyperbolic conservation laws with source terms, Technical Report, Department of Computational and Applied Mathematics, Rice University 2000,TR00-10. To appear in SIAM J. Control Optim. 
[66] S. Ulbrich, Optimal control of nonlinear hyperbolic conservation laws with source terms, Habilitation Thesis, Fakultat fur Mathematik, Technische Universitat Munchen, January 2002.

[67] S. Ulbrich, Adjoint-based derivative computations for the optimal control of discontinuous solutions of hyperbolic conservation laws, Technical Report, Fakultat fur Mathematik, Technische Universitat Munchen September 2001. To appear in Syst. Control Lett. (2002)

[68] J. Vlcek and L. Luksan, Globally convergent variable metric method for nonconvex nondifferentiable unconstrained minimization, J. Optimiz. Theory App.111(2), 407(2001).

[69] Y.J. Wang, H. Matsuhisa and Y. Honda, Loads on lower limb joints and optimal action of muscles for shock reduction, JSME Int. J. C-Mech. SY.42(3), $574(1999)$.

[70] Q. Xu, Generalized adjoint for physical processes with parameterized discontinuities. Part I: Basic issues and heuristic examples Part II: Vector formulations and matching conditions, J. Atmos. Sci. 53(8), 1123(1996).

[71] S. Zhang, X. Zou, J.E. Ahlquist, I.M. Navon and J. Sela, Use of differentiable and nondifferentiable optimization algorithms for variational data assimilation with discontinuous cost functions Mon. Wea. Rev.128(12), 4031(2000).

[72] S. Zhang, X. Zou and J.E. Ahlquist, Examination of numerical results from tangent linear and adjoint of discontinuous nonlinear models, Mon. Wea. Rev. 129, 2791(2001).

[73] X. Zou, I.M. Navon,M. Berger, P.K. Phua,T. Schlick, F.X. LeDimet, Numerical experience with Limited-Memory Quasi-Newton methods for largescale unconstrained nonlinear minimization, SIAM J. Optim.3(3), 582)1993). 


\section{Captions}

\section{TABLES}

- Table (1): Optimization results for the high-resolution model for time $=0.15$ and first set of observations

- Table (2): Optimization results for the high-resolution model for time $=0.24$ and first set of observations

- Table (3): Optimization results for the high-resolution model for time $=0.15$ and second set of observations

- Table (4): Optimization results for the high-resolution model for time $=0.24$ and second set of observations

- Table (5): Optimization results for the artificial viscosity model for time $=0.15$ and first set of observations

- Table (6): Optimization results for the artificial viscosity model for time $=0.24$ and first set of observations

- Table (7): Optimization results for the artificial viscosity model for time $=0.15$ and second set of observations

- Table (8): Optimization results for the artificial viscosity model for time $=0.24$ and second set of observations 


\section{FIGURES}

- Figure (1): The accuracy check for the gradient for the numerical model with dissipation for time $=0.15$ (top) and time $=0.24$ (bottom)

- Figure (2): The accuracy check for the gradient for the high-resolution numerical model for time $=0.15$ (top) and time $=0.24$ (bottom)

- Figure (3): Numerical $(\diamond)$ and analytical (red line) solution of high-resolution model for the shock-tube problem at time $=0.24$ for a) pressure, b) density and c) velocity

- Figure (4): Numerical $(\diamond)$ and analytical (red line) solution of artificial viscosity model for the shock-tube problem at time $=0.24$ for a) pressure, b) density and c) velocity

- Figure (5): Initial guess of high-resolution model( $\diamond)$ and exact observation (red line) for the shock-tube problem at time $=0.24$ using the first set of observations a) pressure, b) density and c) velocity

- Figure (6): Initial guess for artificial viscosity model ( $\diamond)$ and exact observation (red line) for the shock-tube problem at time $=0.24$ using the first set of observations a) pressure, b) density and c) velocity

- Figure (7): Initial guess of high-resolution model( $\diamond)$ and exact observation (red line) for the shock-tube problem at time $=0.24$ using the second set of observations a) pressure, b) density and c) velocity

- Figure (8): Initial guess for artificial viscosity model $(\diamond)$ and exact observation (red line) for the shock-tube problem at time $=0.24$ using the second set of observations a) pressure, b) density and c) velocity

- Figure (9): Evolution of the cost functional and subgradient norm during nonsmooth minimization with the high-resolution model for first set of observations 
(at time $=0.15(\mathrm{a}, \mathrm{b})$ and at time $=0.24(\mathrm{c}, \mathrm{d}))$

- Figure (10): Evolution of the cost functional and subgradient norm during nonsmooth minimization with the high-resolution model for second set of observations (at time $=0.15(\mathrm{a}, \mathrm{b})$ and at time $=0.24(\mathrm{c}, \mathrm{d}))$

- Figure (11): Evolution of the cost functional and subgradient norm during nonsmooth minimization with the high-resolution model at time $=0.24$ for first set $(\mathrm{a}, \mathrm{b})$ and second set of distributed observations (c,d)

- Figure (12): Evolution of the cost functional and subgradient norm during LBFGS minimization with the high-resolution model for time $=0.15$ (first set of observations $(\mathrm{a}, \mathrm{b})$ and second set of observations $(\mathrm{c}, \mathrm{d}))$

- Figure (13): Evolution of the cost functional and gradient norm during nonsmooth minimization with the artificial viscosity model for first set of observations (at time $=0.15(\mathrm{a}, \mathrm{b})$ and at time $=0.24(\mathrm{c}, \mathrm{d}))$

- Figure (14): Evolution of the cost functional and gradient norm during nonsmooth minimization with the artificial viscosity model for second set of observations (at time $=0.15(\mathrm{a}, \mathrm{b})$ and at time $=0.24(\mathrm{c}, \mathrm{d}))$

- Figure (15): Evolution of the cost functional and gradient norm during LBFGS minimization with the artificial viscosity model with weight $=0.0$ for time $=0.15$ (first set of observations (a,b) and second set of observations $(c, d)$ )

- Figure (16): Evolution of the cost functional and gradient norm during LBFGS minimization with the artificial viscosity model with weight $=0.0$ for time $=0.24$ (first set of observations (a,b) and second set of observations $(c, d)$ )

- Figure (17): Evolution of the cost functional and gradient norm during nonsmooth minimization with the artificial viscosity model with weight $=0.0$ for time $=0.15$ (first set of observations (a,b) and second set of observations $(c, d)$ ) 
- Figure (18): Evolution of the cost functional and gradient norm during LBFGS minimization with the artificial viscosity model for time $=0.24$ and second set of observations

- Figure (19): Evolution of the cost functional and gradient norm during LBFGS minimization with the artificial viscosity model for time $=0.15$ (first set of observations $(a, b)$ and second set of observations $(c, d))$

- Figure (20): Desired solution (first set of observations) (red line) and numerical solution $(\diamond)$ after nonsmooth optimization of high-resolution model at time $=0.24$ for a) pressure, b) density and c) velocity

- Figure (21): Desired solution (second set of observations) (red line) and numerical solution $(\diamond)$ after nonsmooth optimization of high-resolution model at time $=0.24$ for a) pressure, b) density and c) velocity

- Figure (22): Desired solution (second set of observations) (red line) and numerical solution $(\diamond)$ after LBFGS optimization of high-resolution model at time $=0.24$ for a) pressure, b) density and c) velocity

- Figure (23): Desired solution (first set of observations) (red line) and numerical solution $(\diamond)$ after nonsmooth optimization of artificial velocity model at time $=0.24$ for a) pressure, b) density and c) velocity

- Figure (24): Desired solution (second set of observations) (red line) and numerical solution $(\diamond)$ after nonsmooth optimization of artificial velocity model at time $=0.24$ for a) pressure, b) density and c) velocity

- Figure (25): Desired solution (first set of observations) (red line) and numerical solution $(\diamond)$ after LBFGS optimization of artificial velocity model at time $=0.24$ for a) pressure, b) density and c) velocity

- Figure (26): Desired solution (second set of observations) (red line) and numerical 
solution $(\diamond)$ after LBFGS optimization of artificial velocity model at time $=0.24$ for a) pressure, b) density and c) velocity

- Figure (27): Desired solution (first set of observations) (red line) and numerical solution $(\diamond)$ after LBFGS optimization (with weight=0.0) of artificial velocity model at time $=0.24$ for a) pressure, b) density and c) velocity

- Figure (28): The result of finding discontinuities (red filled triangles) for the numerical solution of artificial velocity model at time $=0.24$ for a) pressure, b) density and c) velocity

- Figure (29): Numerical $(\diamond)$ and analytical (red line) solution of high-resolution model for the shock-tube problem at time $=0.30$ for a) pressure, b) density and c) velocity

- Figure (30): Evolution of the cost functional and gradient norm during nonsmooth minimization with the initial guess from the LBFGS output for the artificial viscosity model for time $=0.24$ and first set of observations 
Table 1

Optimization results for the high-resolution model for time $=0.15$ and first set of observations

\begin{tabular}{|c|c|c|c|}
\hline Parameter & Desired & LBFGS & PVAR \\
\hline$\rho_{L}$ & 1.1 & 1.10143 & 1.10059 \\
$p_{L}$ & 1.1 & 1.10251 & 1.10187 \\
$\rho_{R}$ & 0.2 & 0.19934 & 0.19942 \\
$p_{R}$ & 0.2 & 0.19865 & 0.19884 \\
\hline
\end{tabular}

Table 2

Optimization results for the high-resolution model for time $=0.24$ and first set of observations

\begin{tabular}{|c|c|c|c|c|c|}
\hline Parameter & Desired & LBFGS & PVAR & LBFGS scaled & PVAR (d.c.) \\
\hline$\rho_{L}$ & 1.1 & Failed & 1.09815 & 1.04032 & 1.10088 \\
$p_{L}$ & 1.1 & Failed & 1.08966 & 0.99664 & 1.10915 \\
$\rho_{R}$ & 0.2 & Failed & 0.19993 & 0.13887 & 0.20122 \\
$p_{R}$ & 0.2 & Failed & 0.19894 & 0.99628 & 0.19886 \\
\hline
\end{tabular}


Table 3

Optimization results for the high-resolution model for time $=0.15$ and second set of observations

\begin{tabular}{|c|c|c|c|}
\hline Parameter & Desired & LBFGS & PVAR \\
\hline$\rho_{L}$ & 1.2 & 1.20161 & 1.20052 \\
$p_{L}$ & 1.2 & 1.20342 & 1.20278 \\
$\rho_{R}$ & 0.3 & 0.29712 & 0.29752 \\
$p_{R}$ & 0.3 & 0.29973 & 0.29953 \\
\hline
\end{tabular}

Table 4

Optimization results for the high-resolution model for time $=0.24$ and second set of observations

\begin{tabular}{|c|c|c|c|c|c|}
\hline Parameter & Desired & LBFGS & PVAR & LBFGS scaled & PVAR (d.c.) \\
\hline$\rho_{L}$ & 1.2 & 1.03479 & 1.19406 & 1.38023 & 1.20689 \\
$p_{L}$ & 1.2 & 0.85757 & 1.19203 & 0.84461 & 1.20698 \\
$\rho_{R}$ & 0.3 & 0.35072 & 0.30308 & 0.37357 & 0.30294 \\
$p_{R}$ & 0.3 & 0.25325 & 0.29946 & 0.26728 & 0.29962 \\
\hline
\end{tabular}


Table 5

Optimization results for the artificial viscosity model for time $=0.15$ and first set of observations

\begin{tabular}{|c|c|c|c|c|c|}
\hline Parameter & Desired & LBFGS & LBFGS (w=0) & LBFGS (w=25) & PVAR \\
\hline$\rho_{L}$ & 1.1 & 1.09712 & 1.10031 & Failed & 1.09685 \\
$p_{L}$ & 1.1 & 1.09947 & 1.10459 & Failed & 1.09933 \\
$\rho_{R}$ & 0.2 & 0.20432 & 0.20514 & Failed & 0.20439 \\
$p_{R}$ & 0.2 & 0.19756 & 0.19786 & Failed & 0.19782 \\
\hline
\end{tabular}

Table 6

Optimization results for the artificial viscosity model for time $=0.24$ and first set of observations

\begin{tabular}{|c|c|c|c|c|c|c|c|}
\hline Parameter & Desired & LBFGS & LBFGS & LBFGS & PVAR & PVAR & LBFGS \\
& 1.1 & 1.02638 & 1.09742 & Failed & 1.09737 & 1.09741 & 1.03685 \\
$\rho_{L}$ & 1.1 & 1.00347 & 1.10173 & Failed & 1.09966 & 1.09961 & 0.96042 \\
$p_{L}$ & 0.2 & 0.18012 & 0.20004 & Failed & 0.20357 & 0.20344 & 0.13276 \\
$\rho_{R}$ & 0.2 & 0.19296 & 0.20154 & Failed & 0.19874 & 0.19867 & 0.35517 \\
\hline
\end{tabular}


Table 7

Optimization results for the artificial viscosity model for time $=0.15$ and second set of observations

\begin{tabular}{|c|c|c|c|c|c|}
\hline Parameter & Desired & LBFGS & LBFGS (w=0) & LBFGS (w=25) & PVAR \\
\hline$\rho_{L}$ & 1.2 & 1.19784 & 1.19327 & Failed & 1.19768 \\
$p_{L}$ & 1.2 & 1.19856 & 1.18962 & Failed & 1.19778 \\
$\rho_{R}$ & 0.3 & 0.30582 & 0.29983 & Failed & 0.30583 \\
$p_{R}$ & 0.3 & 0.29714 & 0.30134 & Failed & 0.29702 \\
\hline
\end{tabular}

Table 8

Optimization results for the artificial viscosity model for time $=0.24$ and second set of observations

\begin{tabular}{|c|c|c|c|c|c|}
\hline Parameter & Desired & LBFGS & LBFGS (w=0) & LBFGS (w=25) & PVAR \\
\hline$\rho_{L}$ & 1.2 & 1.19832 & 1.19872 & Failed & 1.19764 \\
$p_{L}$ & 1.2 & 1.19846 & 1.19641 & Failed & 1.19825 \\
$\rho_{R}$ & 0.3 & 0.30615 & 0.30373 & Failed & 0.30527 \\
$p_{R}$ & 0.3 & 0.29891 & 0.29778 & Failed & 0.29735 \\
\hline
\end{tabular}



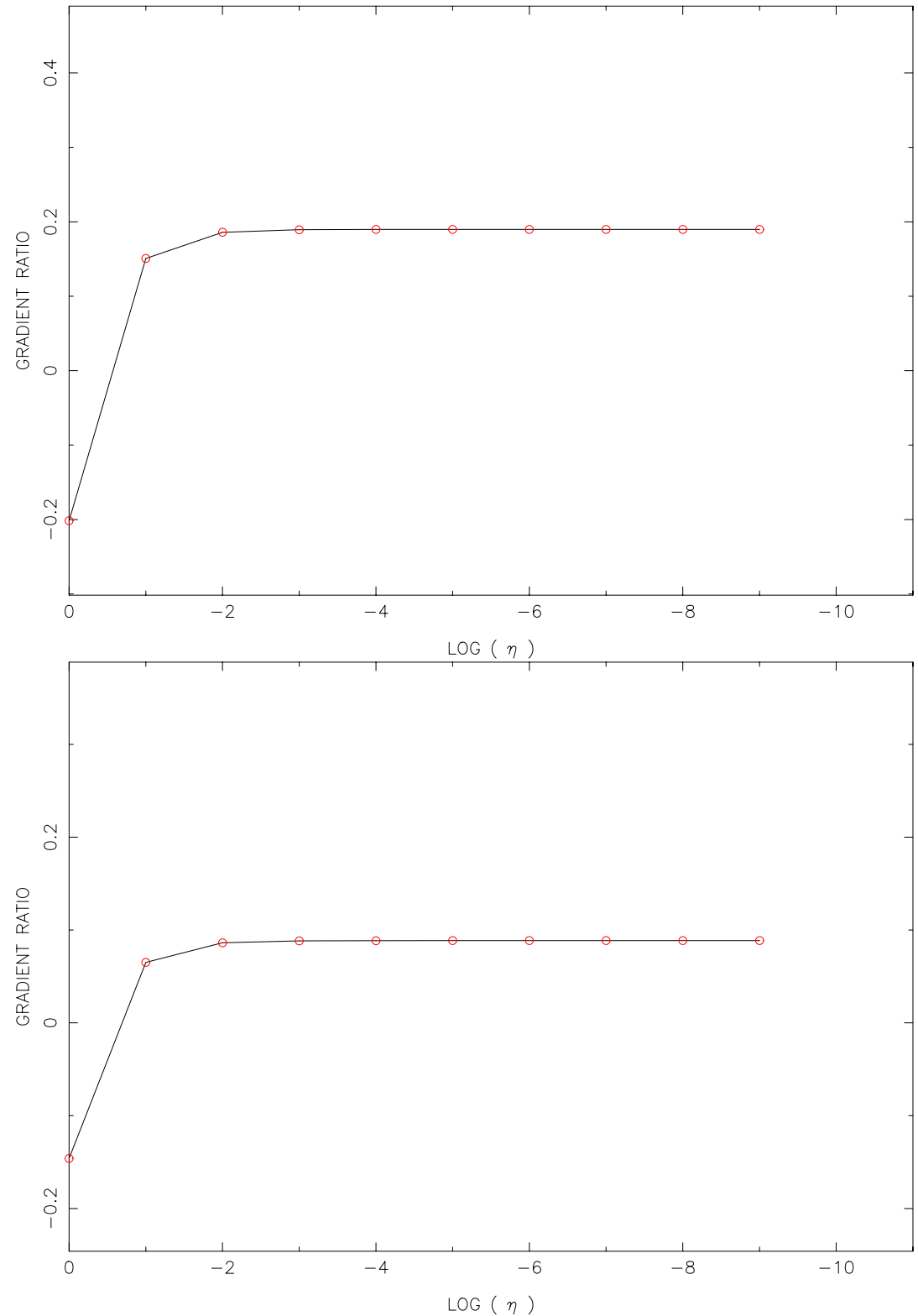

Fig. 1. The accuracy check for the gradient for the numerical model with dissipation for time $=0.15$ (top) and time $=0.24$ (bottom) 

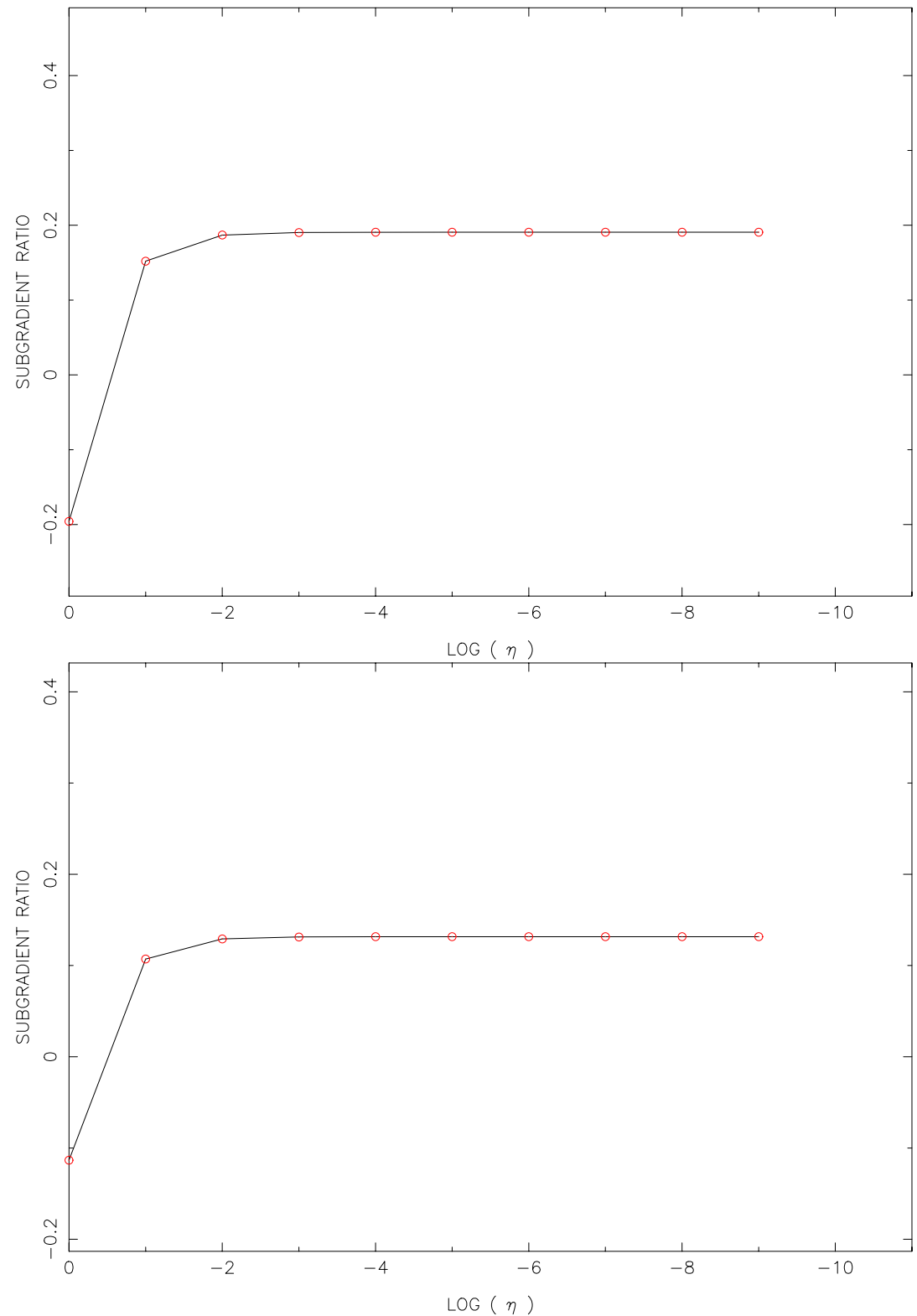

Fig. 2. The accuracy check for the gradient for the high-resolution numerical model for time $=0.15$ (top) and time $=0.24$ (bottom) 


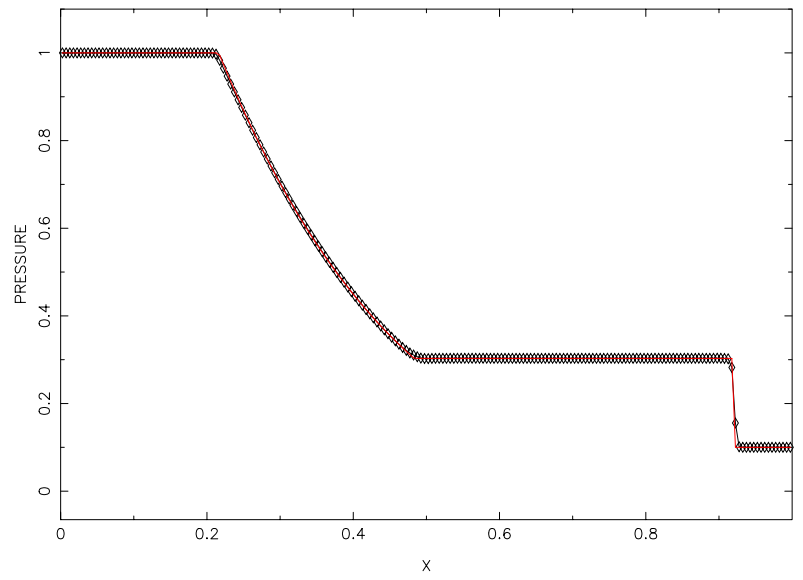

(a)

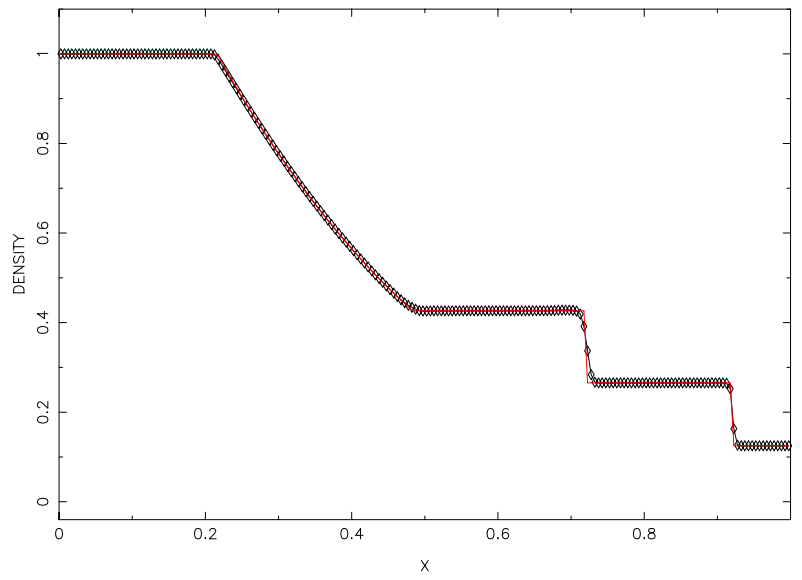

(b)

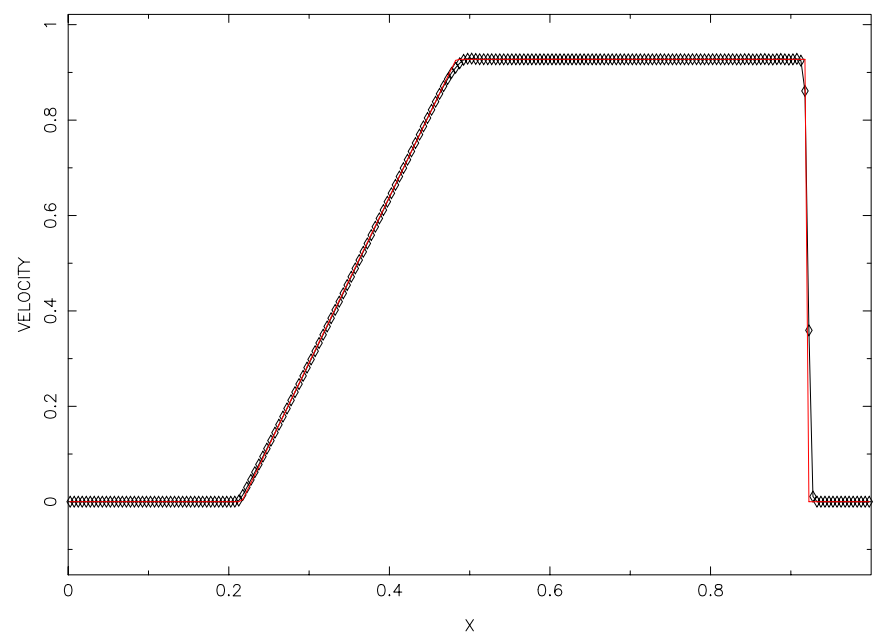

(c)

Fig. 3. Numerical $(\diamond)$ and analytical (red line) solution of high-resolution model for the shock-tube problem at time $=0.24$ for a) pressure, b) density and c) velocity. 


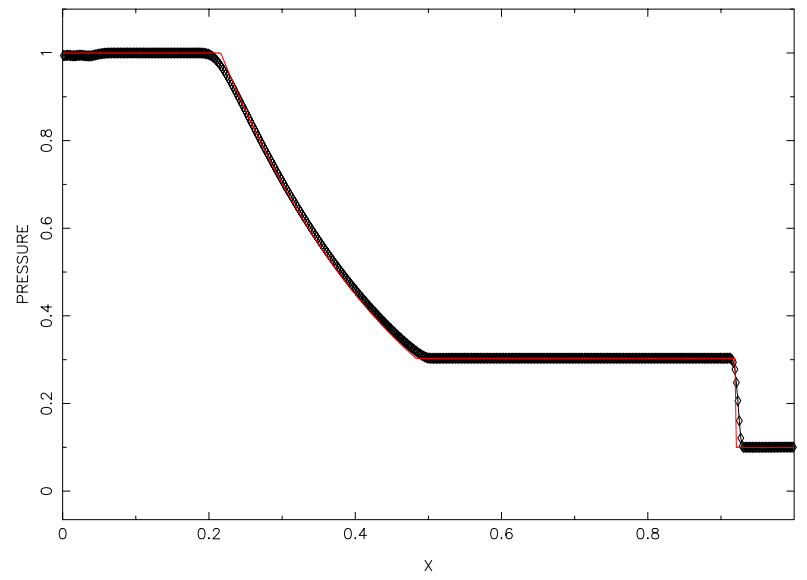

(a)

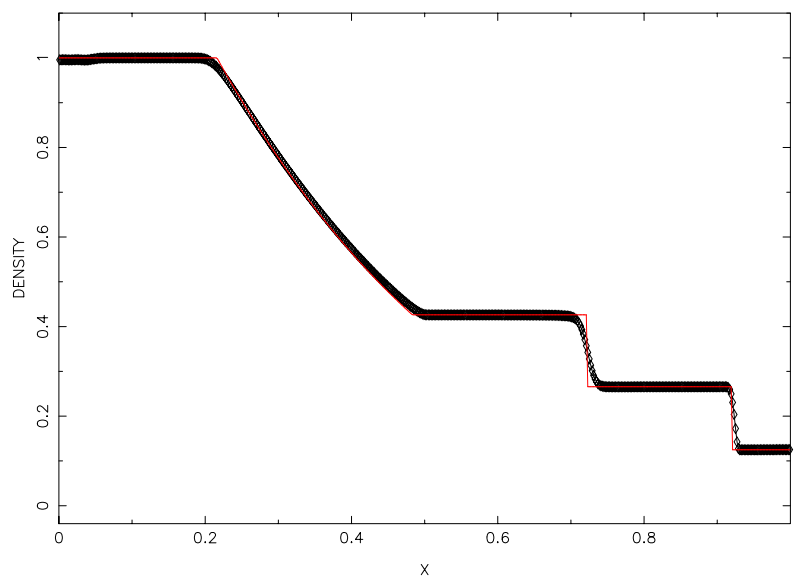

(b)

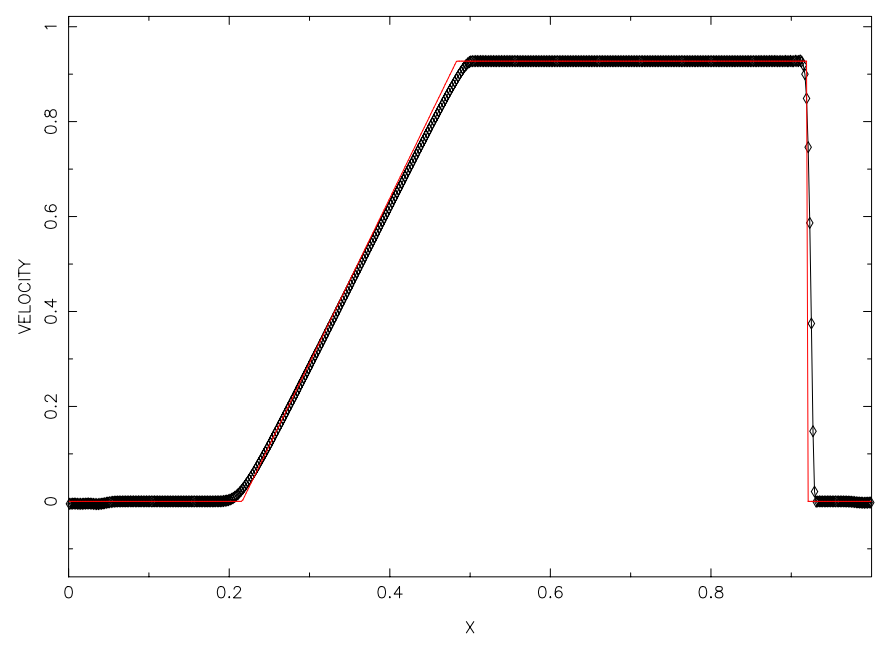

(c)

Fig. 4. Numerical $(\diamond)$ and analytical (red line) solution of artificial viscosity model for the shock-tube problem at time $=0.24$ for a) pressure, b) density and c) velocity. 


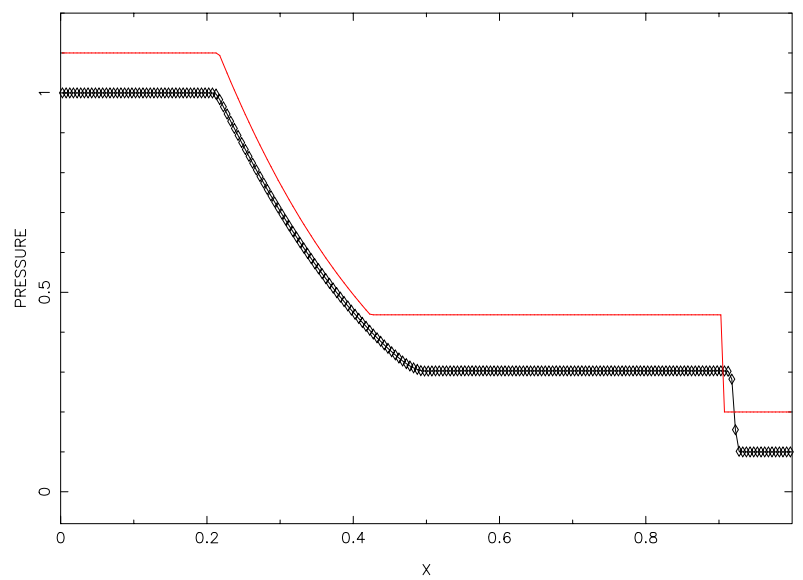

(a)

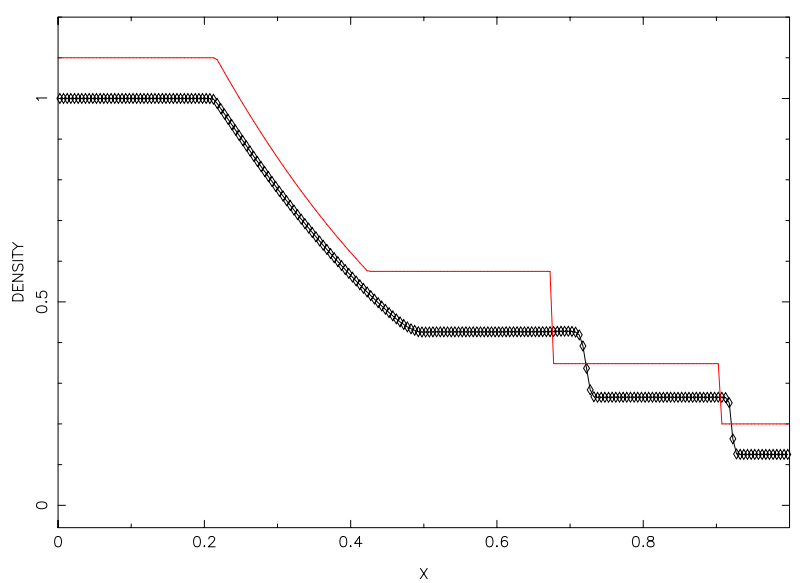

(b)

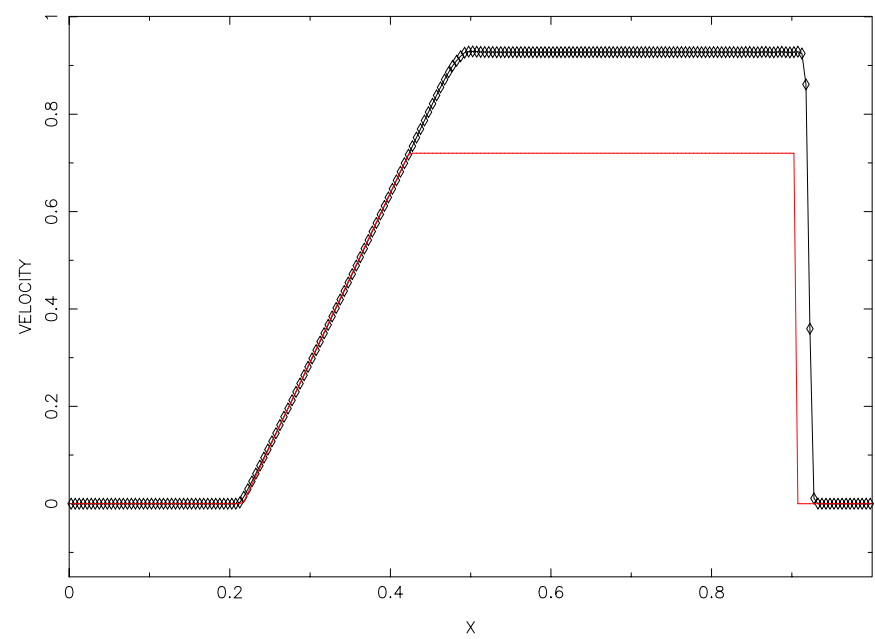

(c)

Fig. 5. Initial guess of high-resolution model $(\diamond)$ and exact observation (red line) for the shock-tube problem at time $=0.24$ using the first set of observations a) pressure, b) density and c) velocity. 


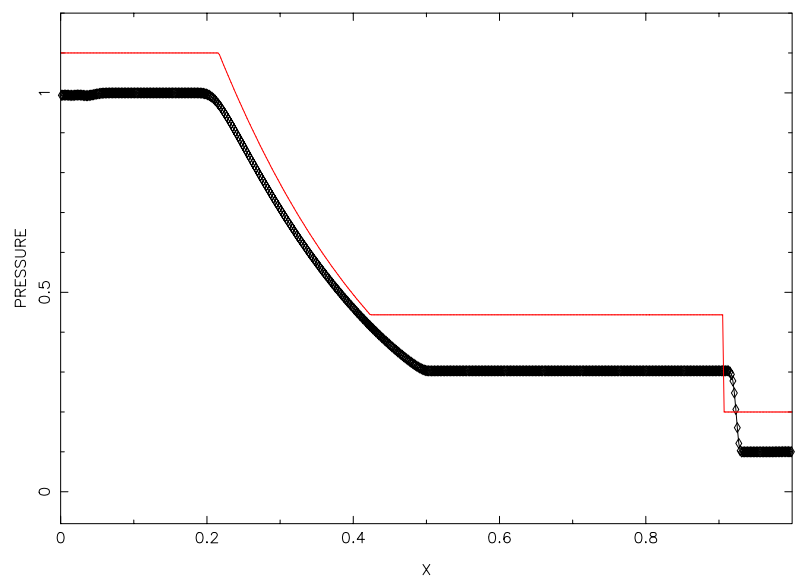

(a)

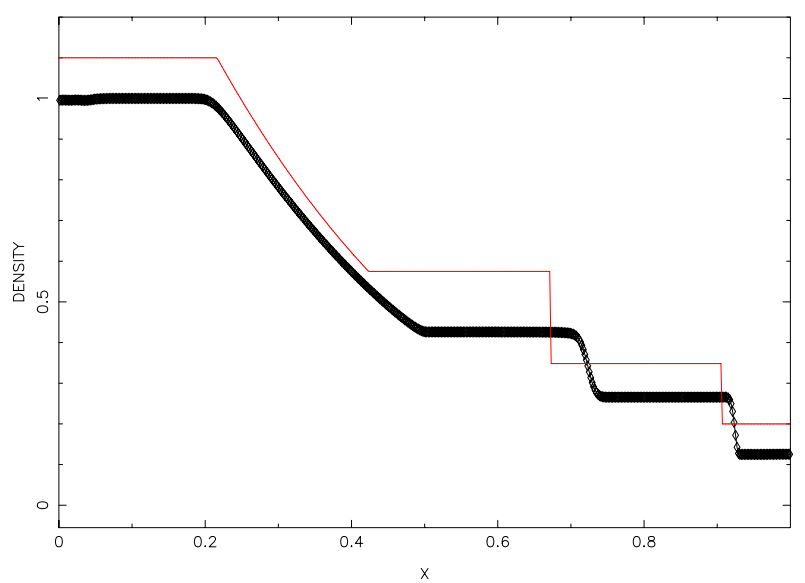

(b)

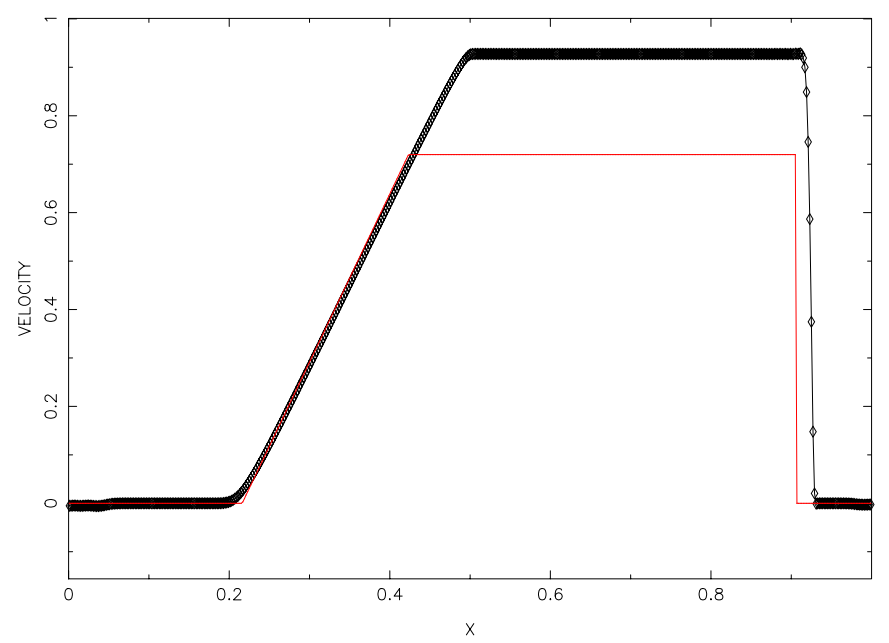

(c)

Fig. 6. Initial guess for artificial viscosity model $(\diamond)$ and exact observation (red line) for the shock-tube problem at time $=0.24$ using the first set of observations a) pressure, b) density and c) velocity. 


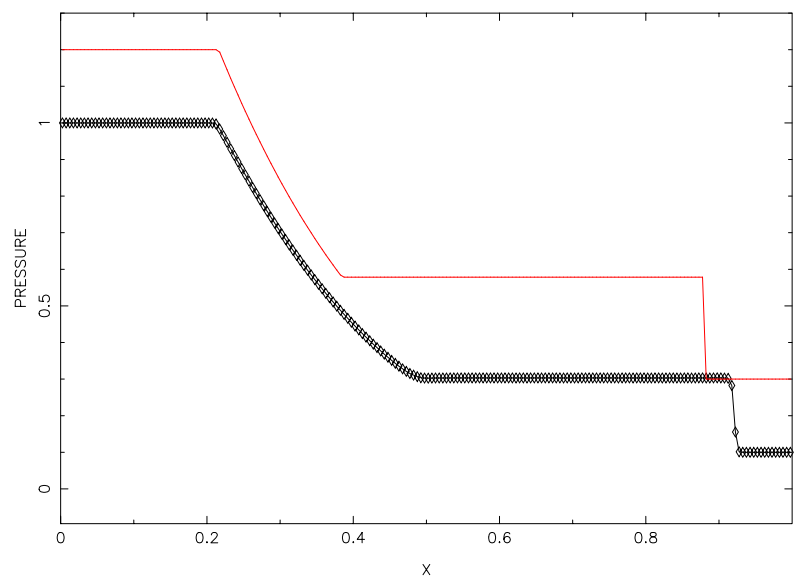

(a)

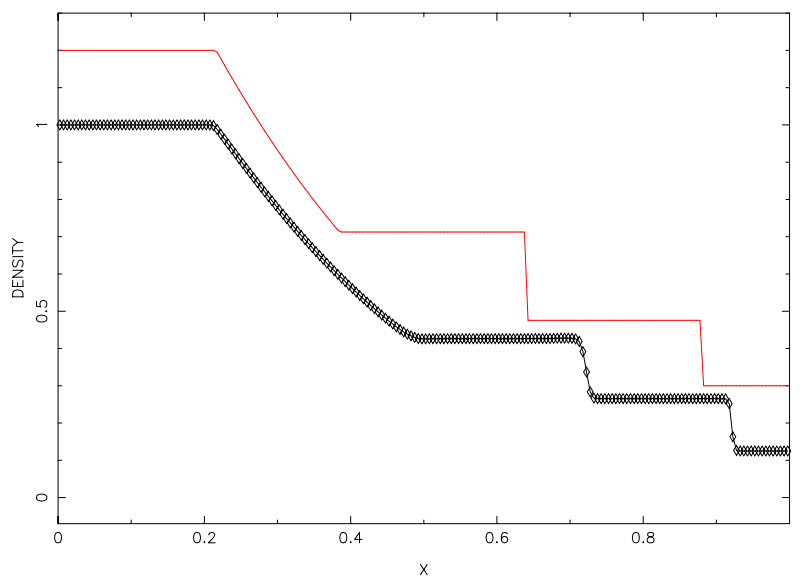

(b)

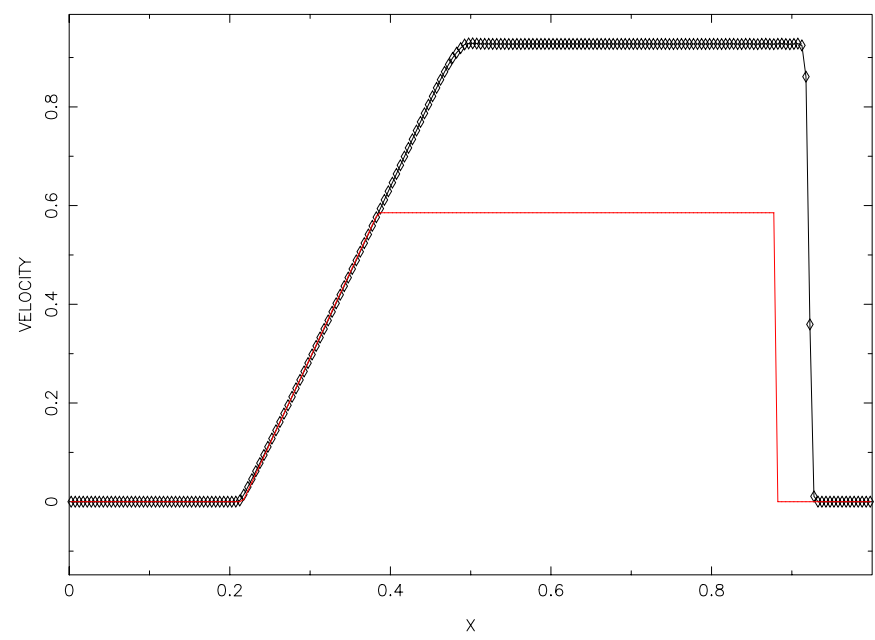

(c)

Fig. 7. Initial guess of high-resolution $\operatorname{model}(\diamond)$ and exact observation (red line) for the shock-tube problem at time $=0.24$ using the second set of observations a) pressure, b) density and c) velocity. 


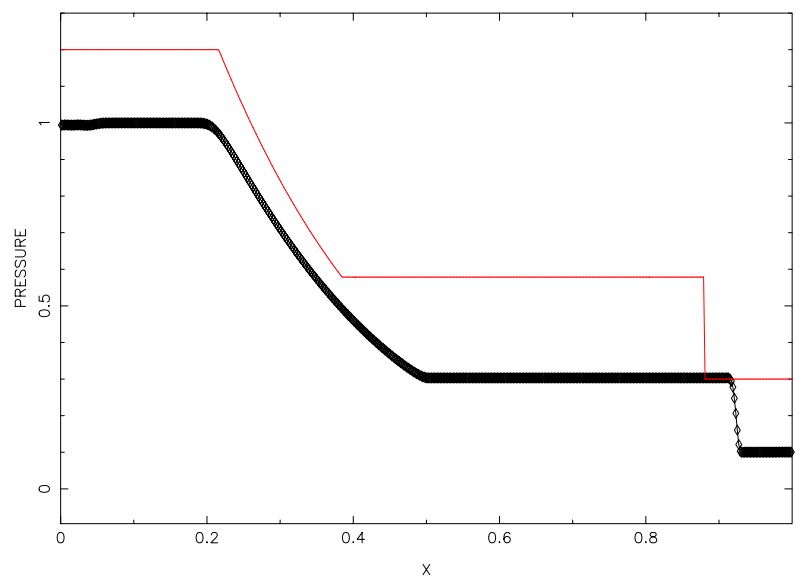

(a)

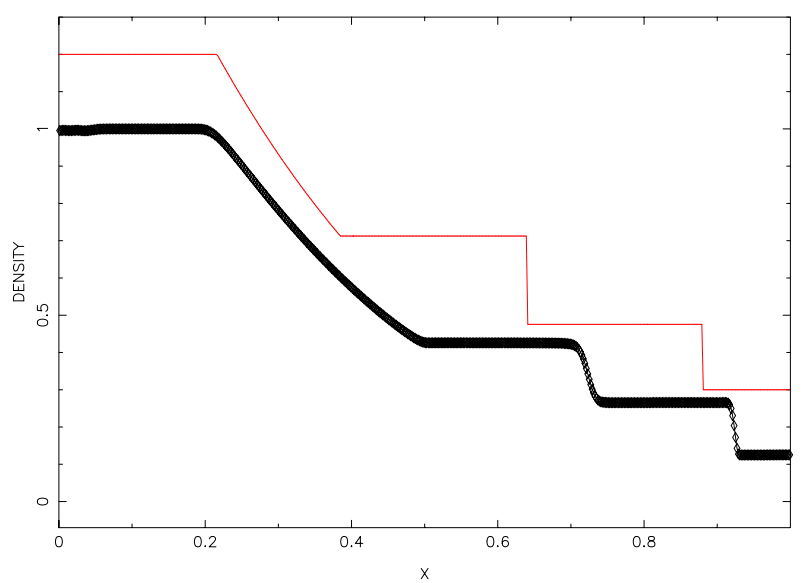

(b)

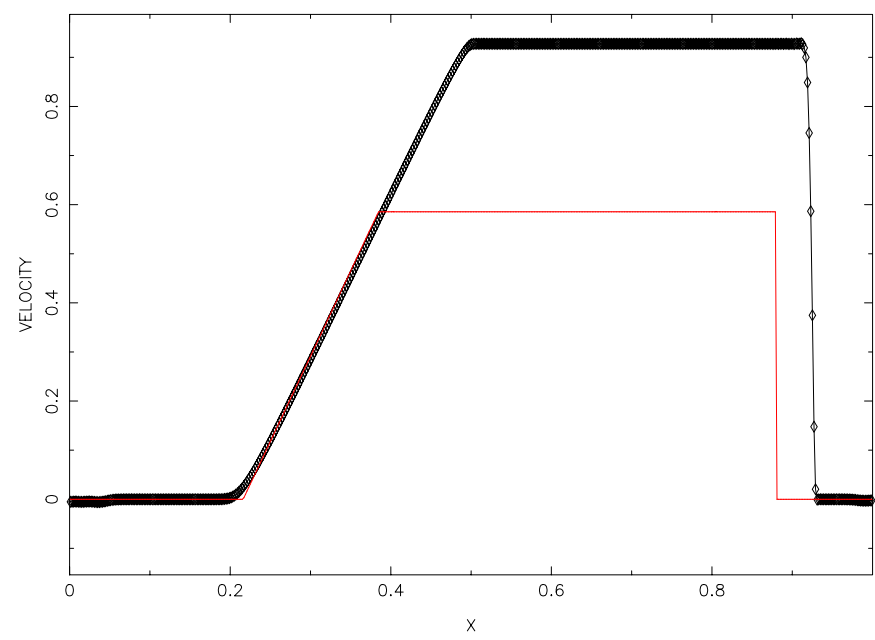

(c)

Fig. 8. Initial guess for artificial viscosity model $(\diamond)$ and exact observation (red line) for the shock-tube problem at time $=0.24$ using the second set of observations a) pressure, b) density and c) velocity. 


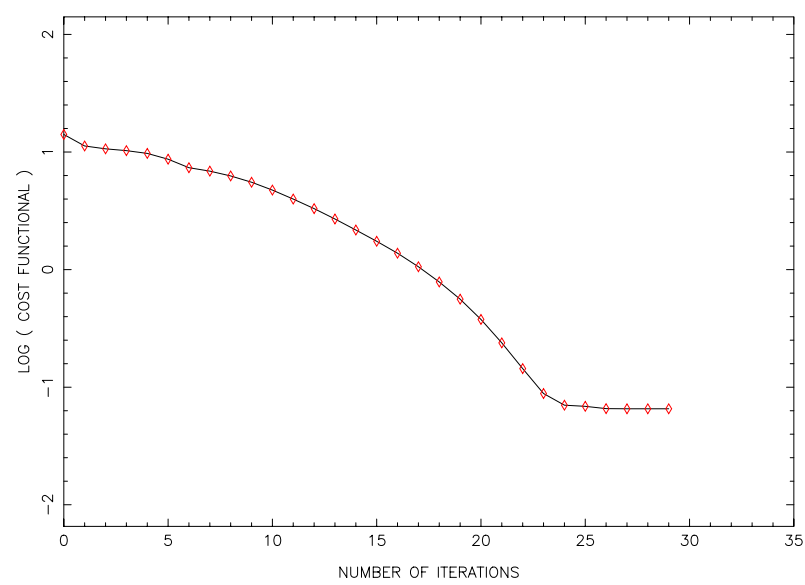

(a)

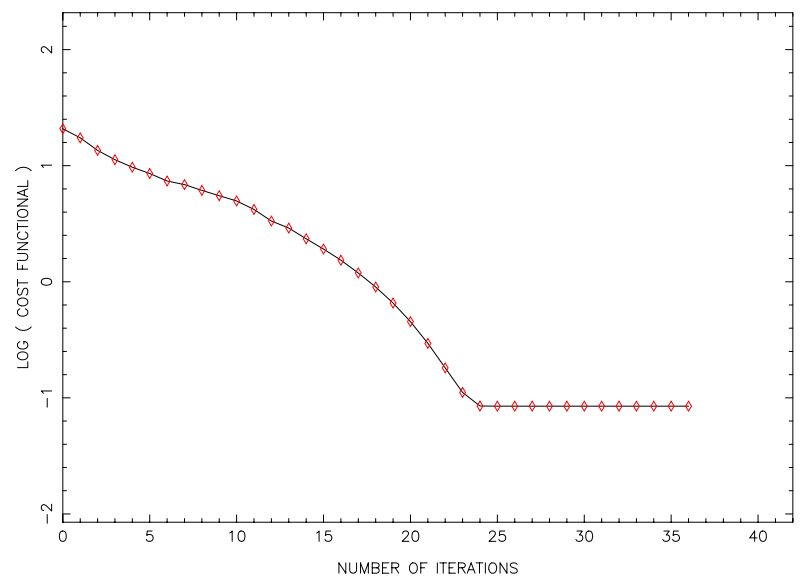

(c)

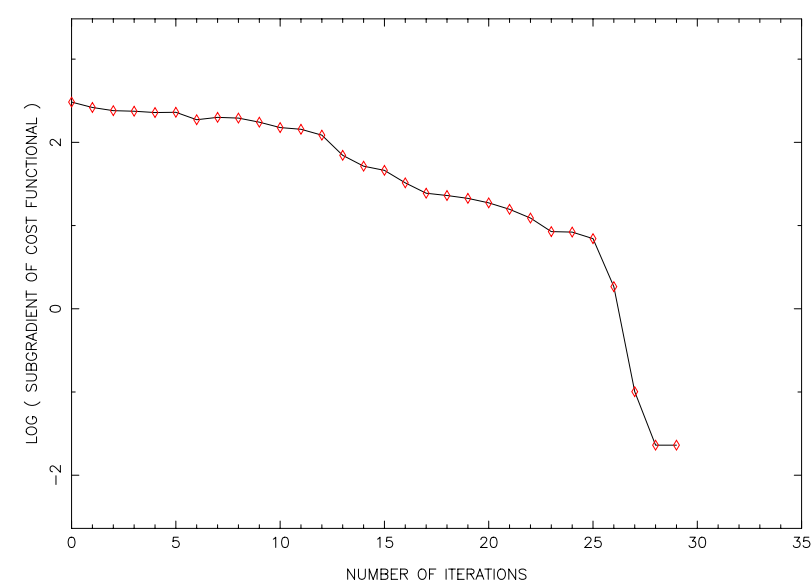

(b)

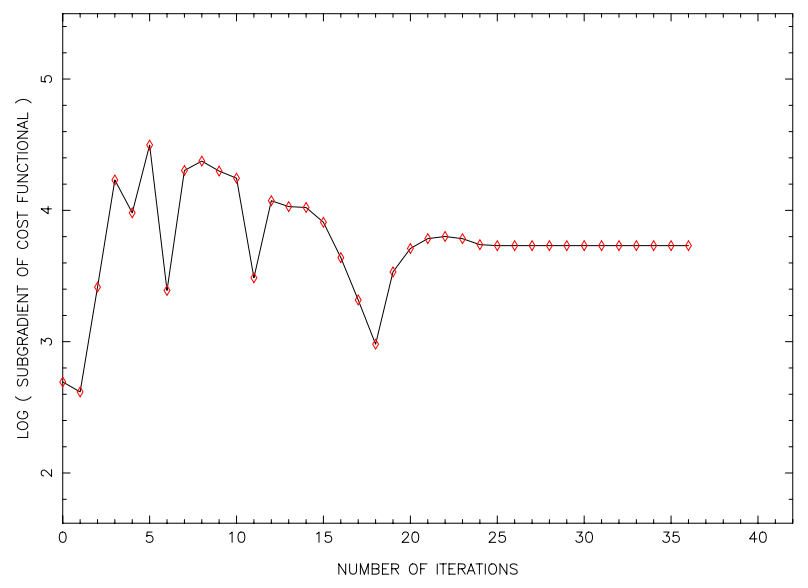

(d)

Fig. 9. Evolution of the cost functional and subgradient norm during nonsmooth minimization with the high-resolution model for first set of observations (at time $=0.15(\mathrm{a}, \mathrm{b})$ and at time $=0.24(\mathrm{c}, \mathrm{d}))$. 


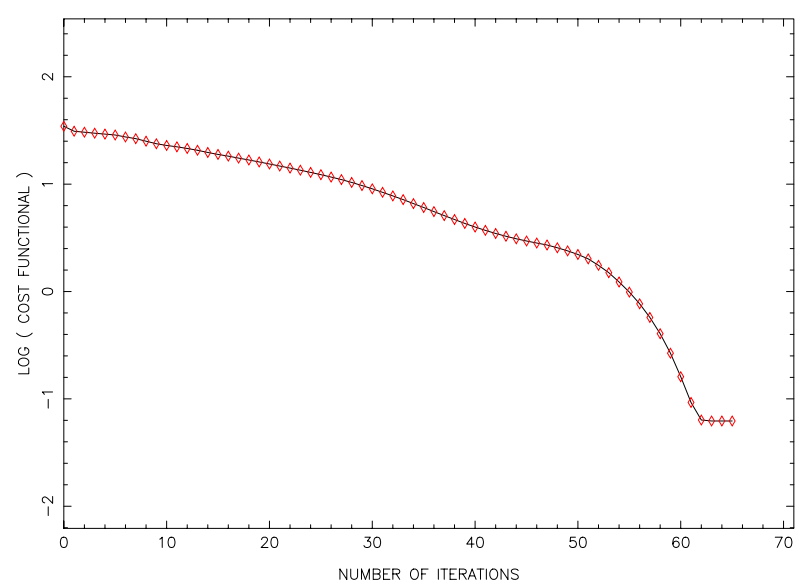

(a)

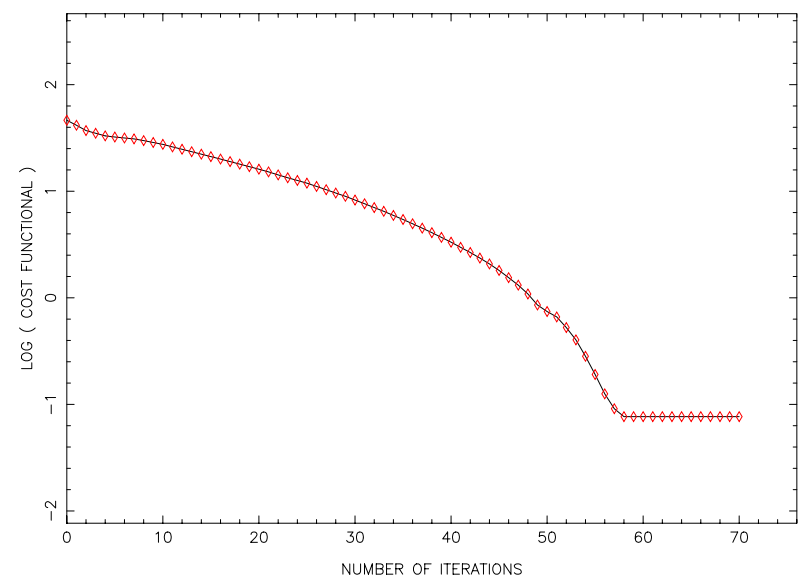

(c)

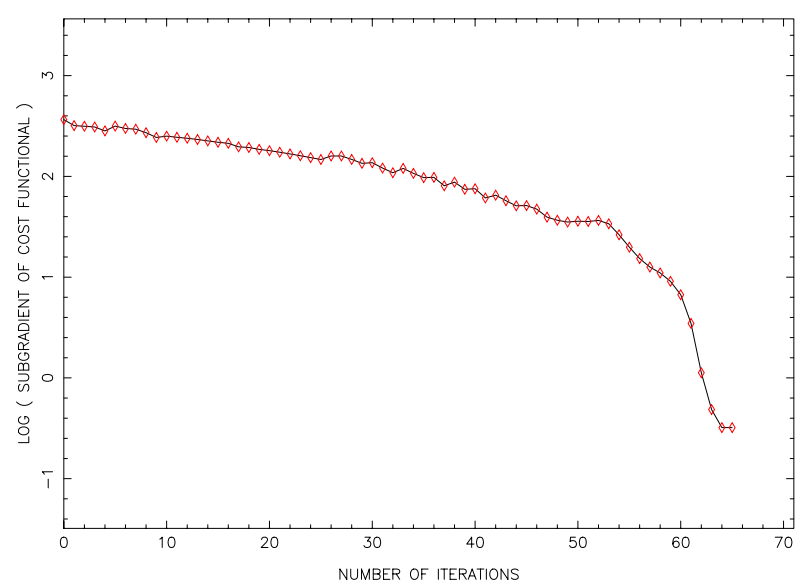

(b)

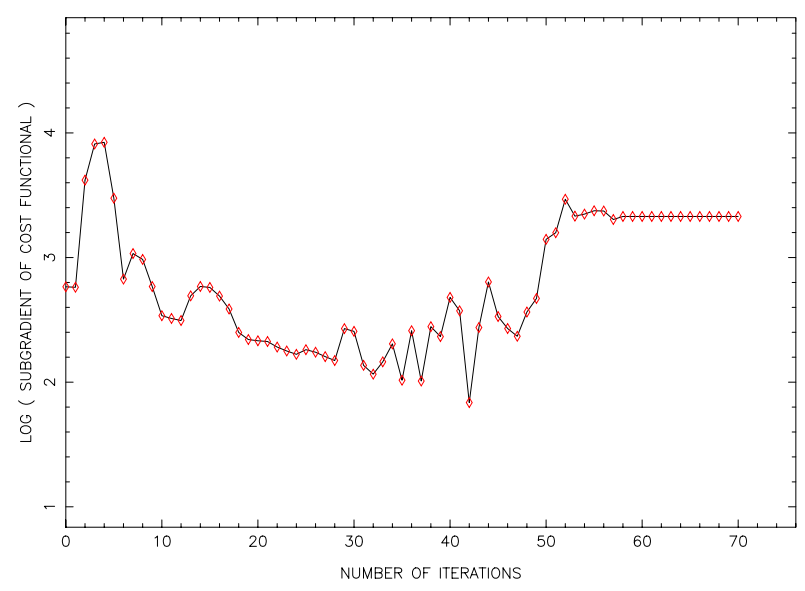

(d)

Fig. 10. Evolution of the cost functional and subgradient norm during nonsmooth minimization with the high-resolution model for second set of observations (at time $=0.15(\mathrm{a}, \mathrm{b})$ and at time $=0.24(\mathrm{c}, \mathrm{d}))$. 


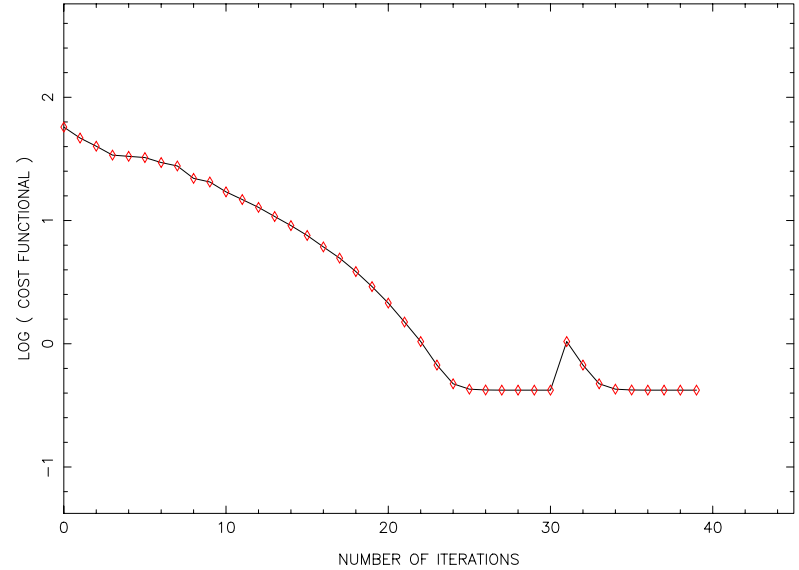

(a)

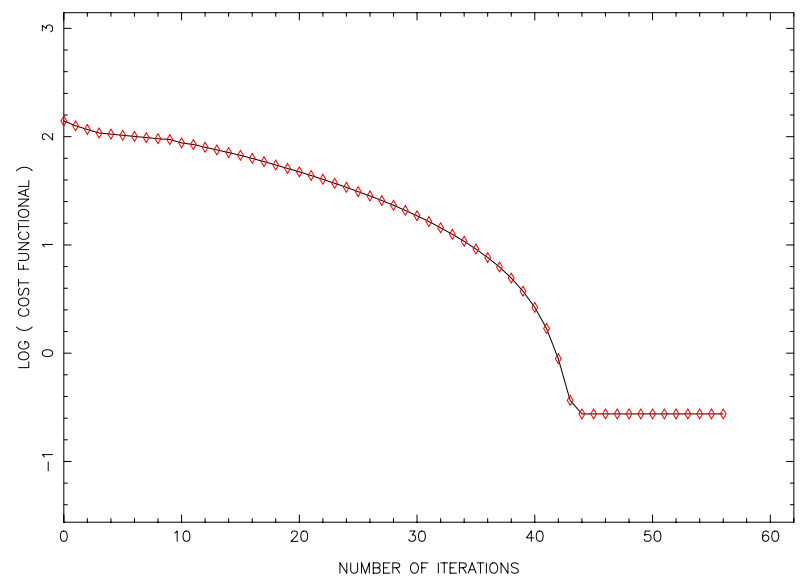

(c)

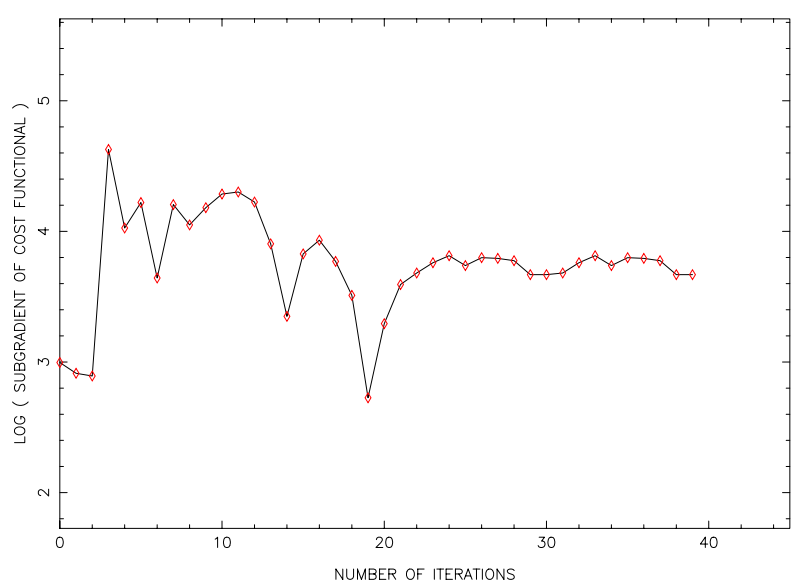

(b)

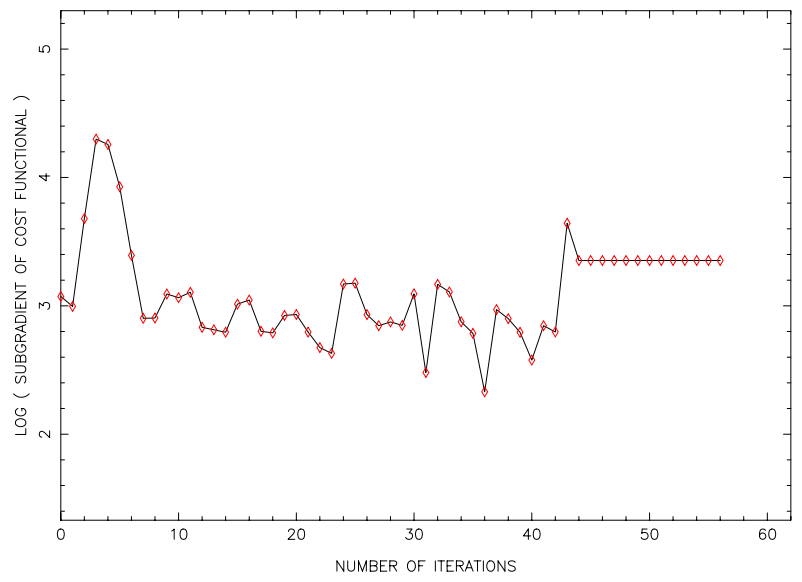

(d)

Fig. 11. Evolution of the cost functional and subgradient norm during nonsmooth minimization with the high-resolution model at time $=0.24$ for first set(a,b) and second set of distributed observations $(c, d)$. 


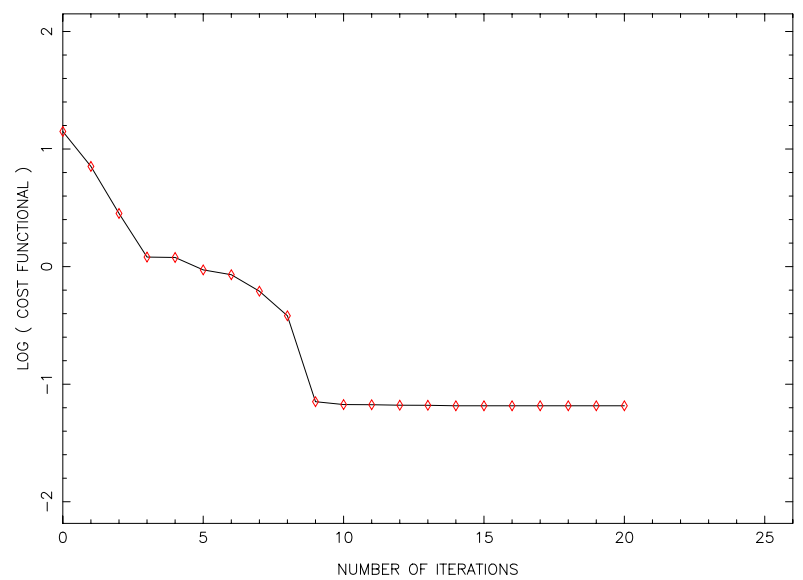

(a)

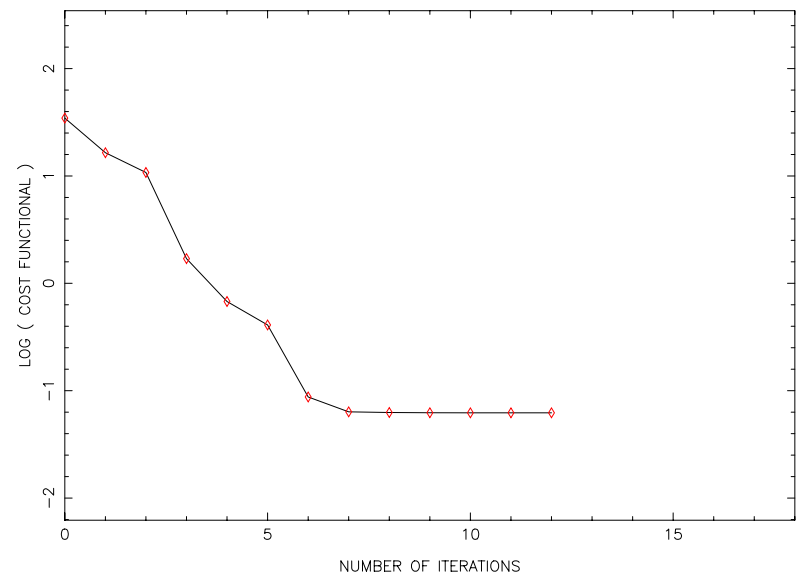

(c)

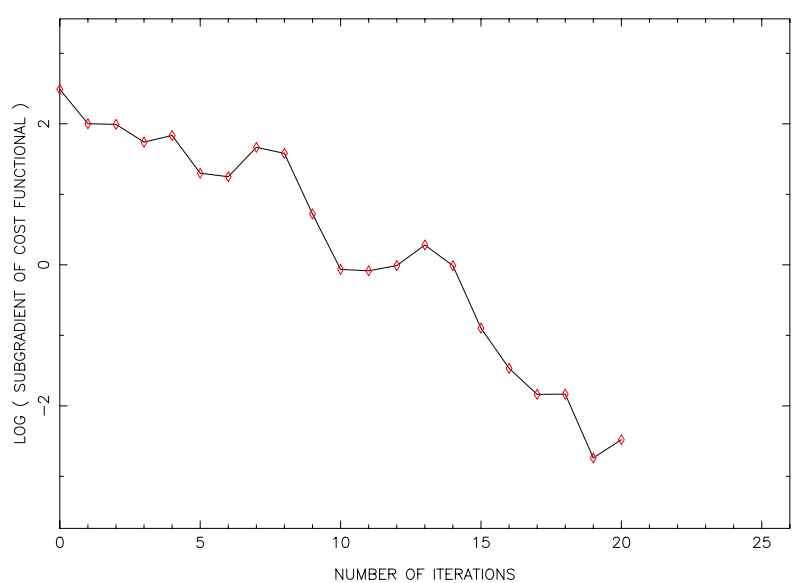

(b)

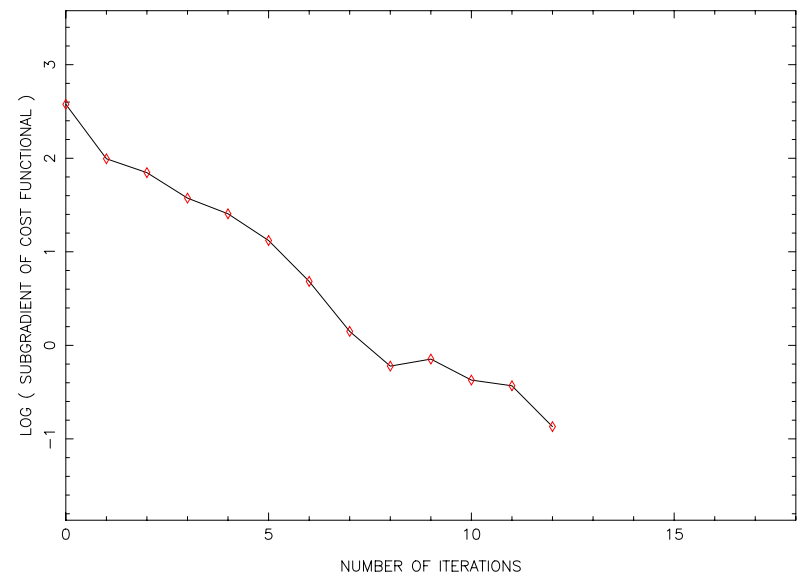

(d)

Fig. 12. Evolution of the cost functional and subgradient norm during LBFGS minimization with the high-resolution model for time $=0.15$ (first set of observations $(a, b)$ and second set of observations $(c, d))$. 


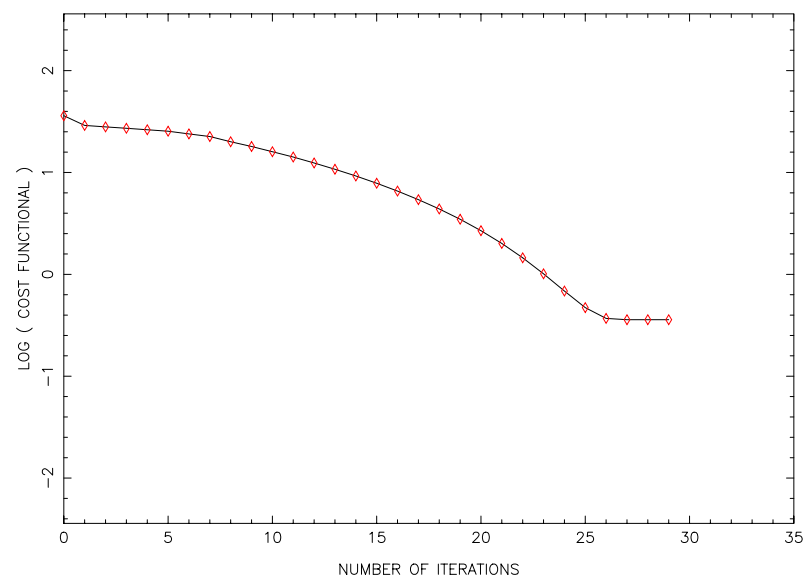

(a)

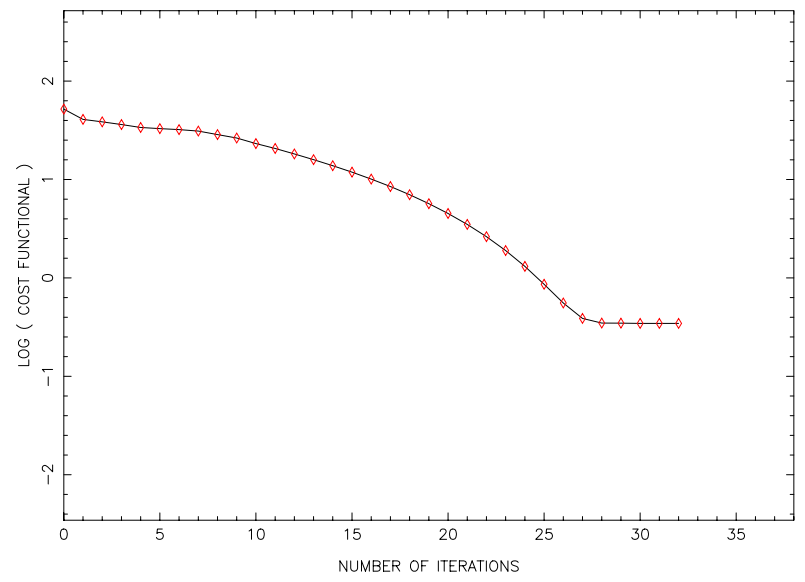

(c)

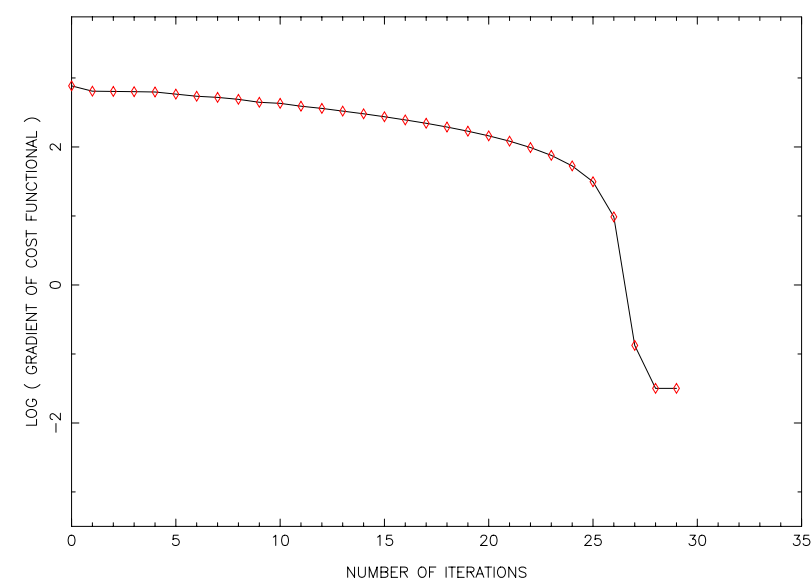

(b)

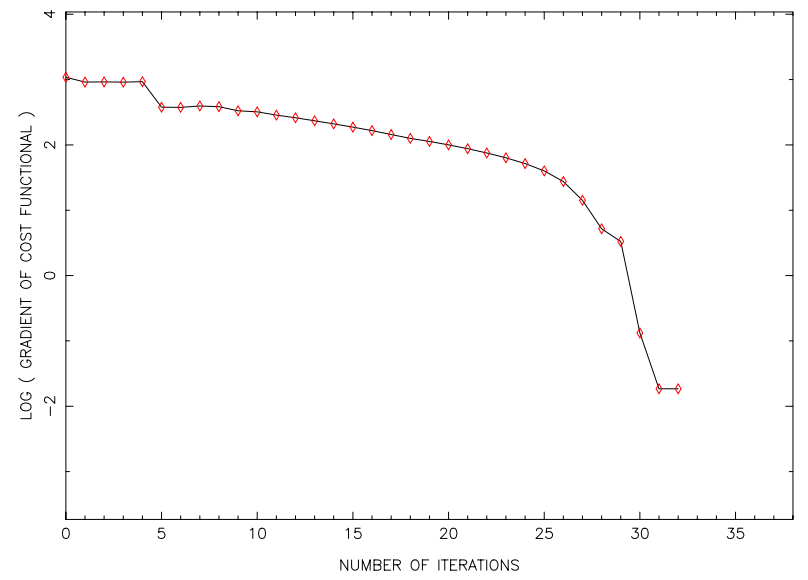

(d)

Fig. 13. Evolution of the cost functional and gradient norm during nonsmooth minimization with the artificial viscosity model for first set of observations (at time $=0.15$ $(\mathrm{a}, \mathrm{b})$ and at time $=0.24(\mathrm{c}, \mathrm{d}))$. 


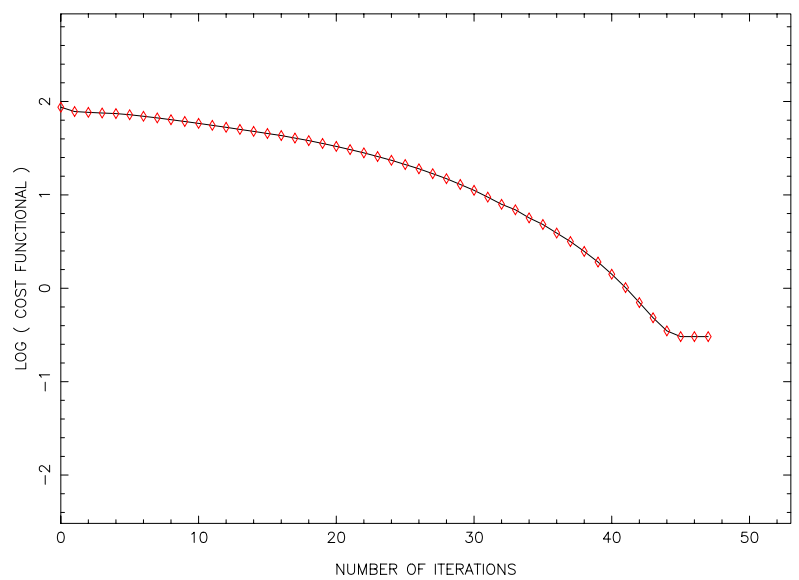

(a)

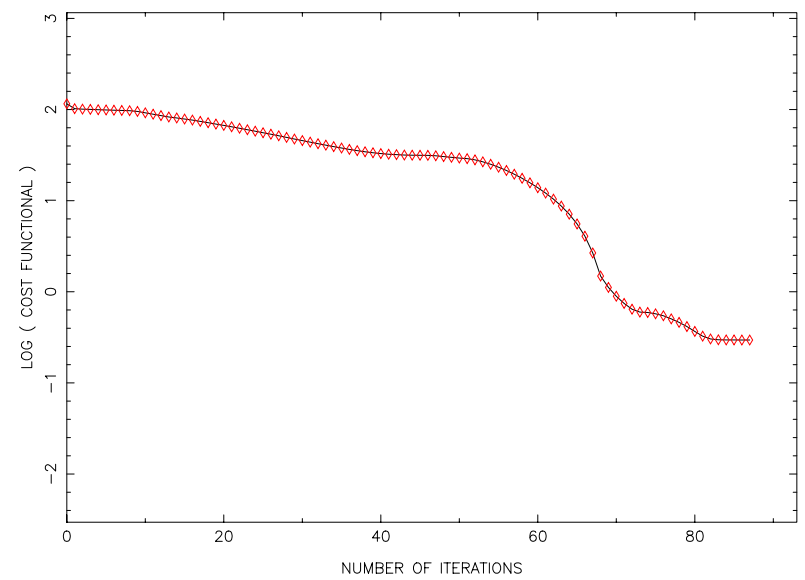

(c)

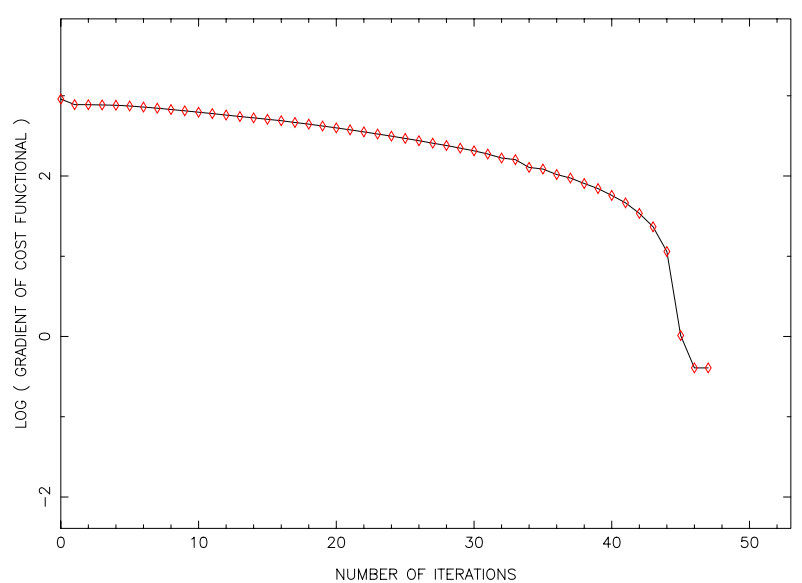

(b)

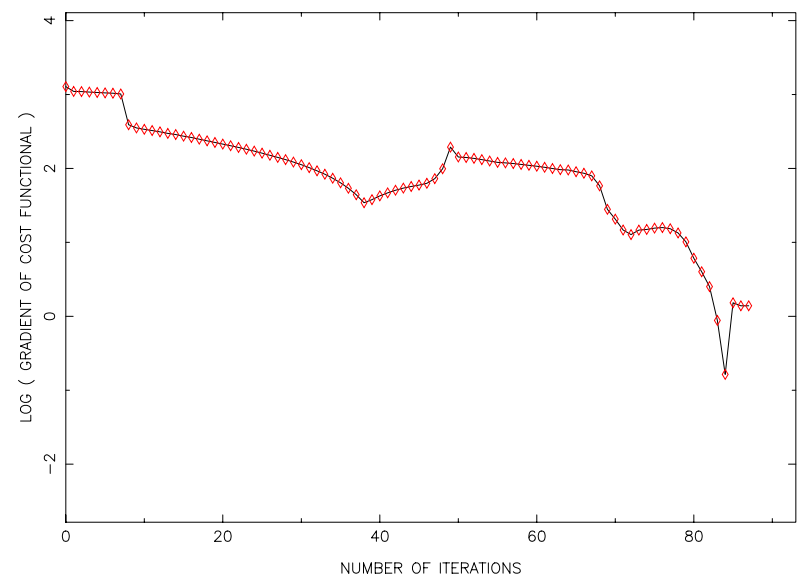

(d)

Fig. 14. Evolution of the cost functional and gradient norm during nonsmooth minimization with the artificial viscosity model for second set of observations (at time $=0.15(\mathrm{a}, \mathrm{b})$ and at time $=0.24(\mathrm{c}, \mathrm{d}))$. 


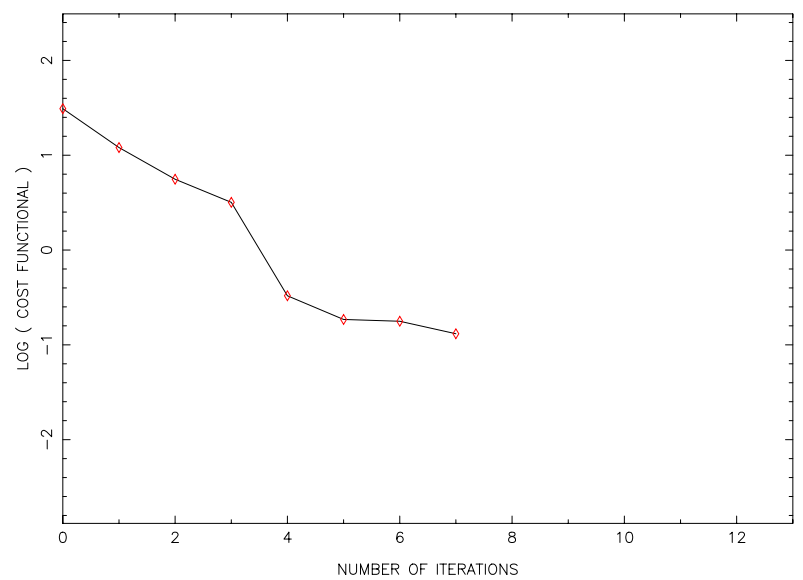

(a)

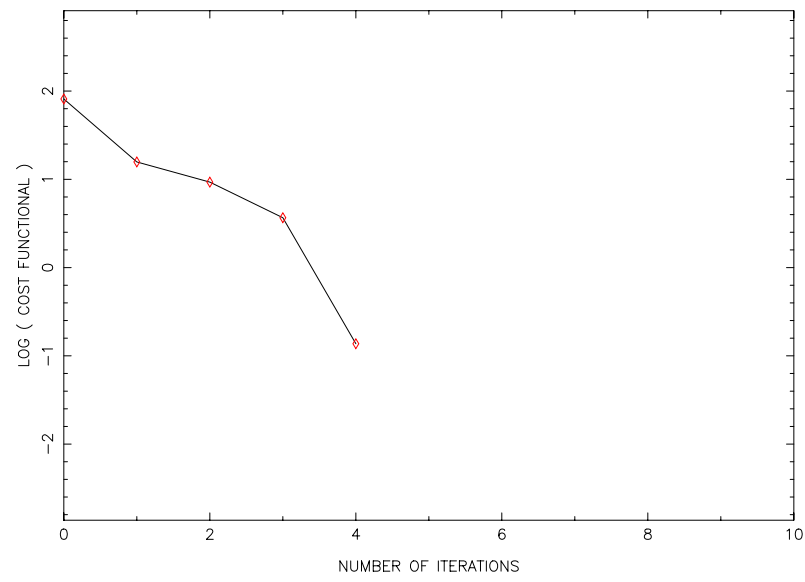

(c)

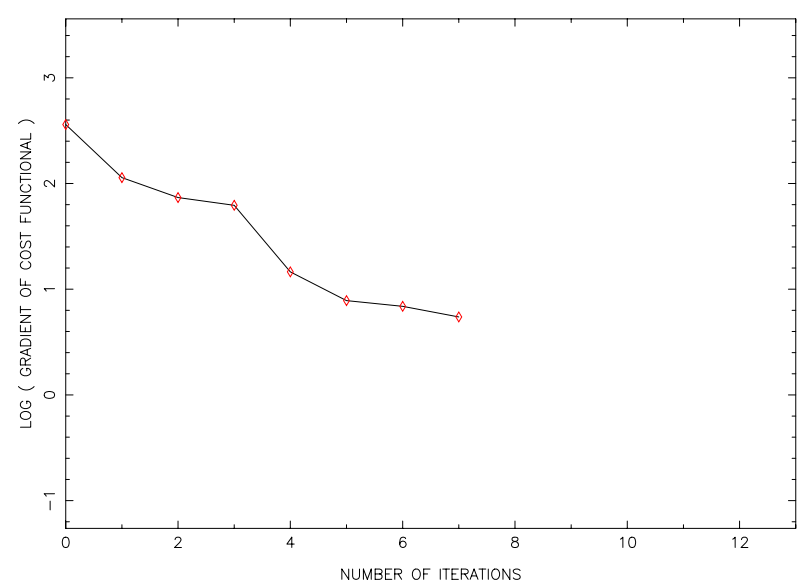

(b)

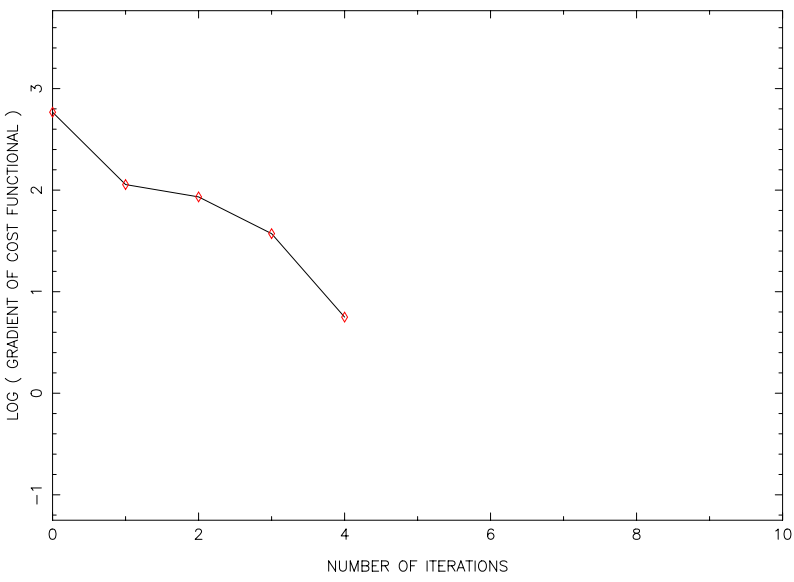

(d)

Fig. 15. Evolution of the cost functional and gradient norm during LBFGS minimization with the artificial viscosity model with weight $=0.0$ for time $=0.15$ (first set of observations (a,b) and second set of observations $(\mathrm{c}, \mathrm{d}))$. 


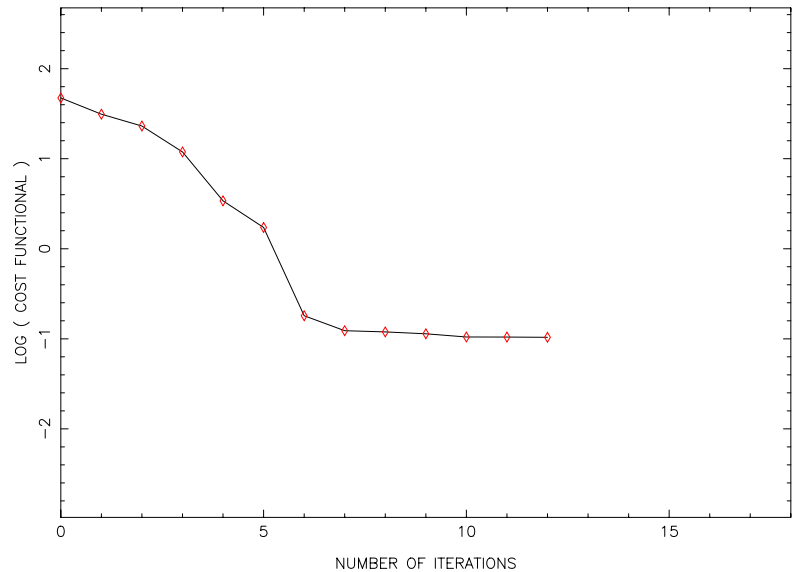

(a)

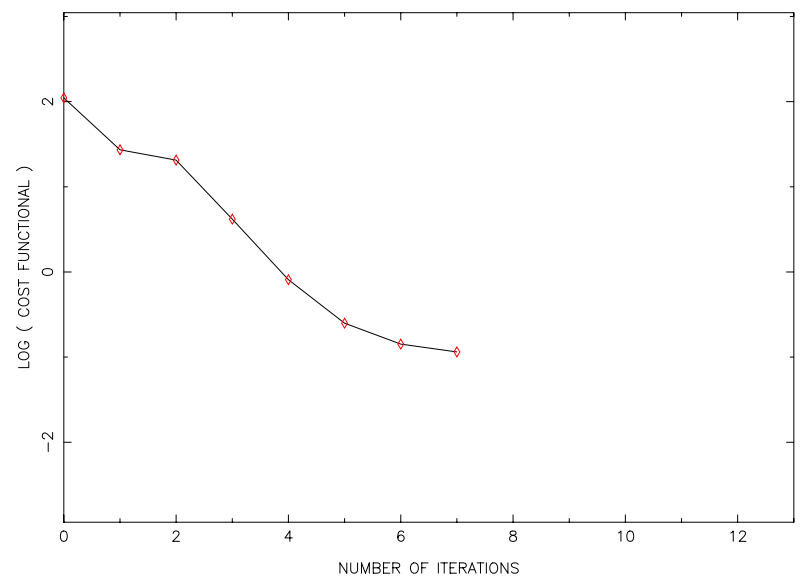

(c)

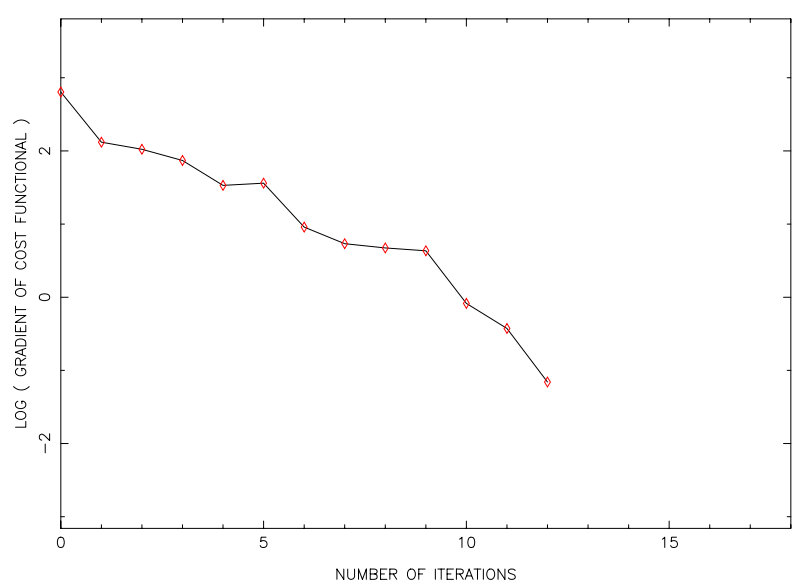

(b)

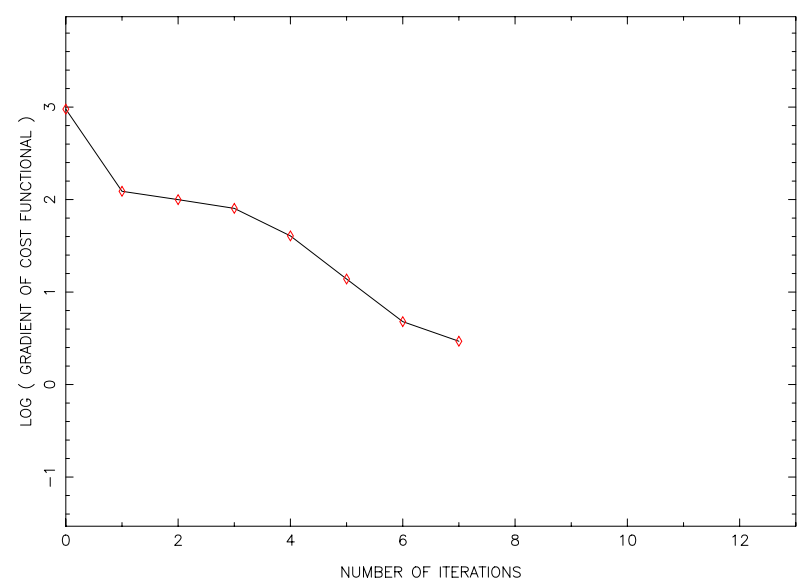

(d)

Fig. 16. Evolution of the cost functional and gradient norm during LBFGS minimization with the artificial viscosity model with weight $=0.0$ for time $=0.24$ (first set of observations (a,b) and second set of observations $(\mathrm{c}, \mathrm{d}))$. 


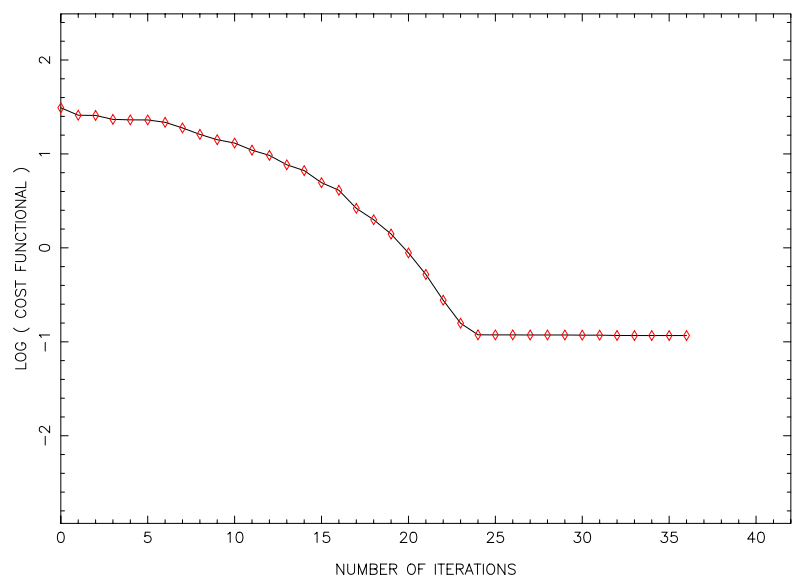

(a)

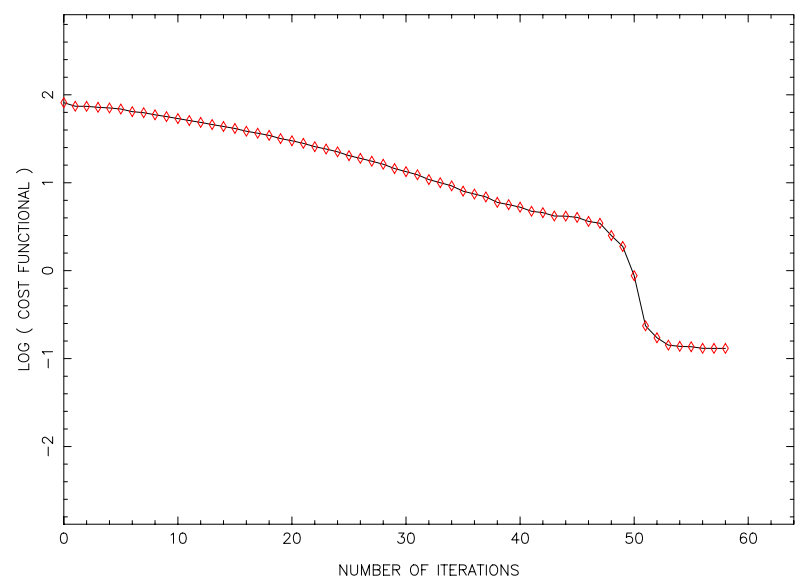

(c)

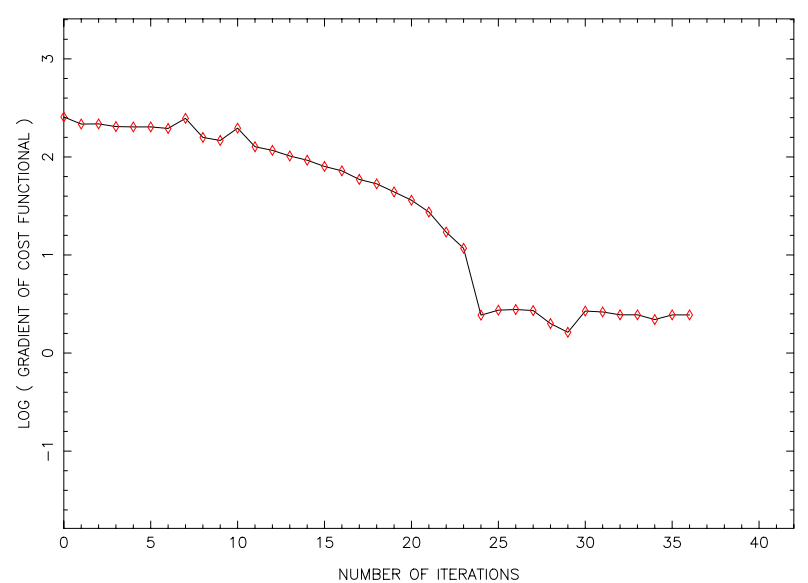

(b)

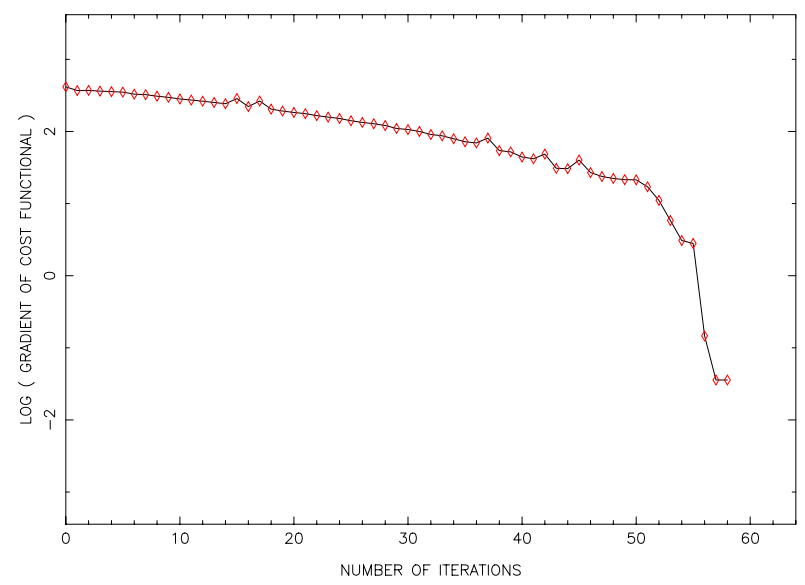

(d)

Fig. 17. Evolution of the cost functional and gradient norm during nonsmooth minimization with the artificial viscosity model with weight $=0.0$ for time $=0.15$ (first set of observations (a,b) and second set of observations (c,d)). 

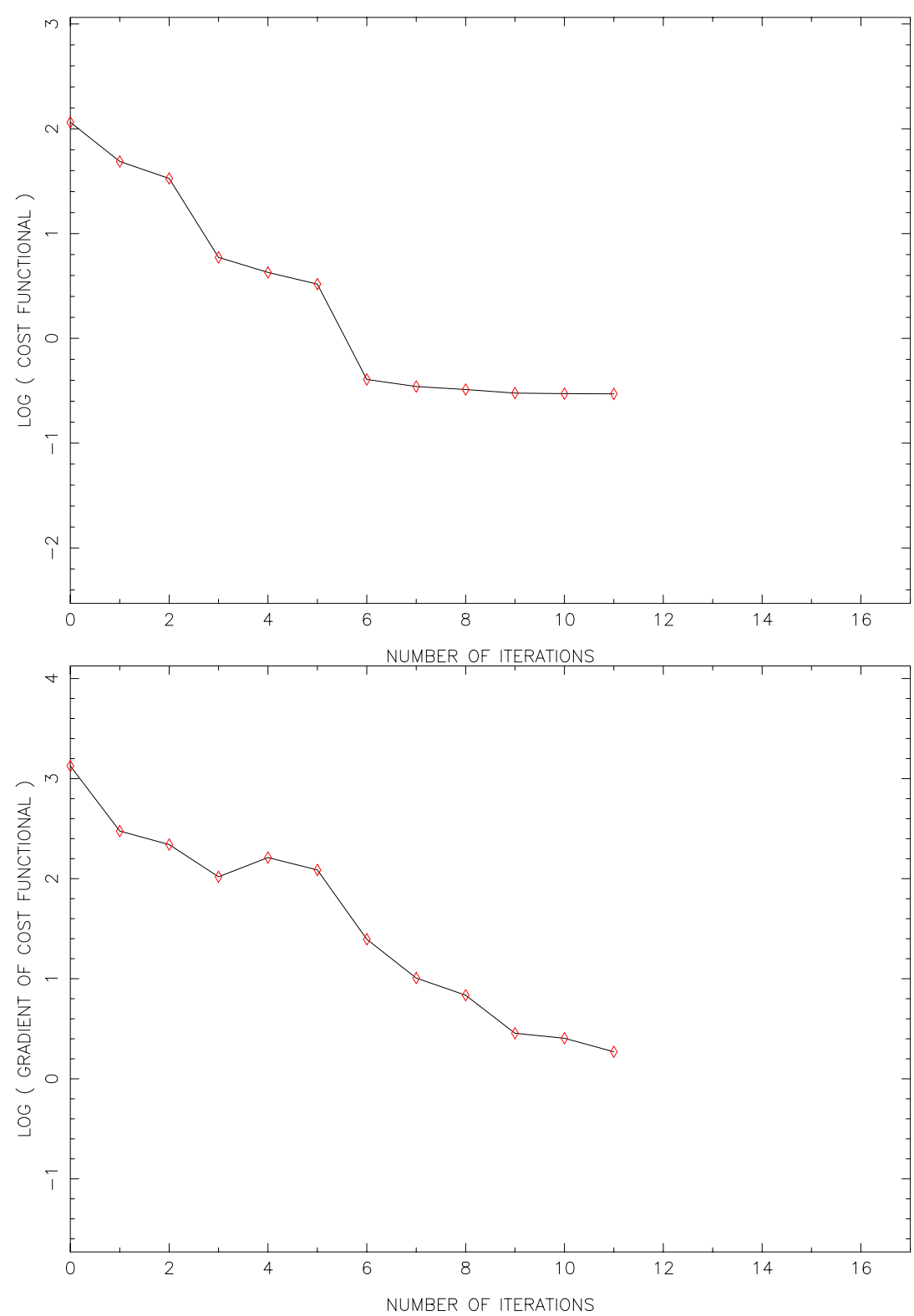

Fig. 18. Evolution of the cost functional and gradient norm during LBFGS minimization with the artificial viscosity model for time $=0.24$ and second set of observations 


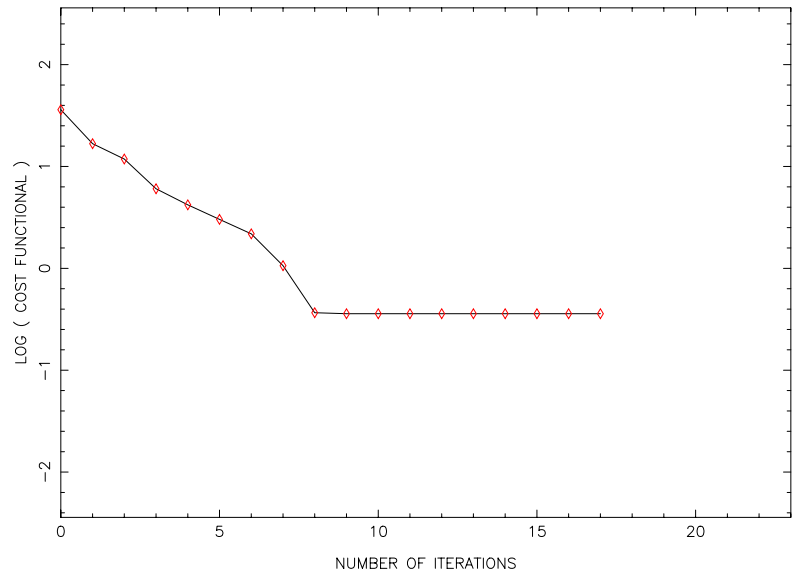

(a)

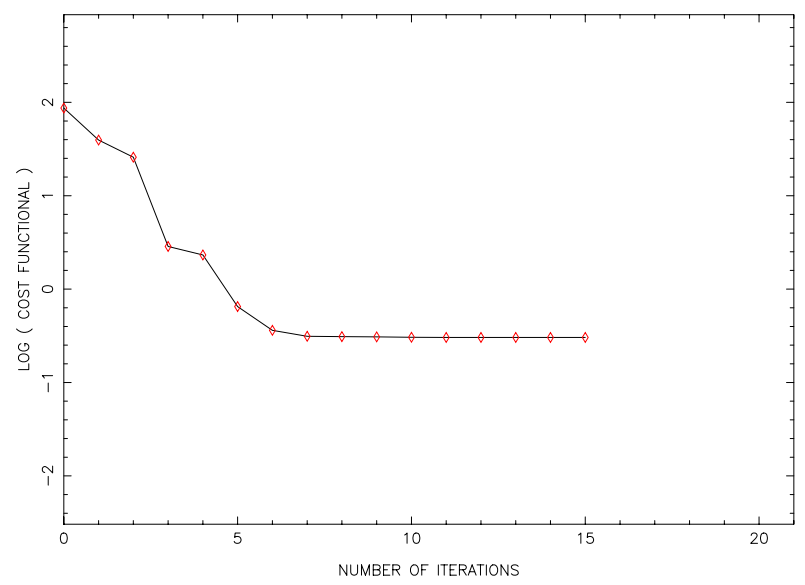

(c)

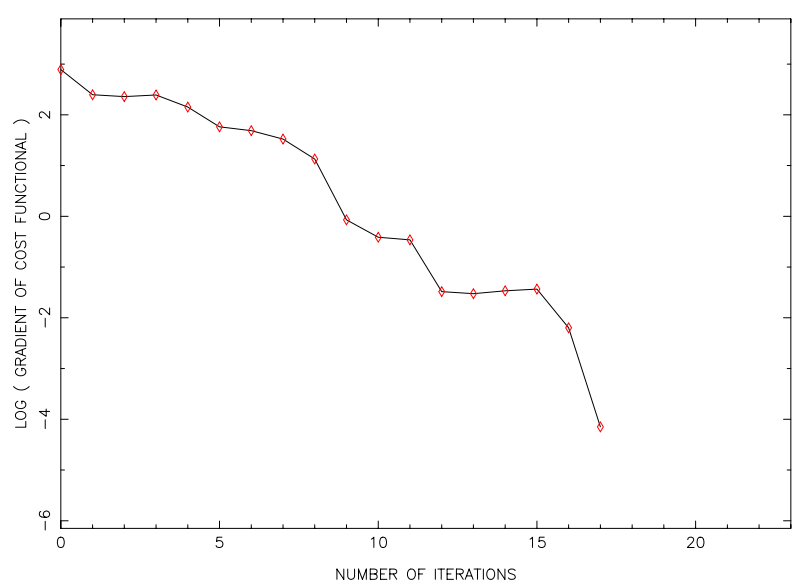

(b)

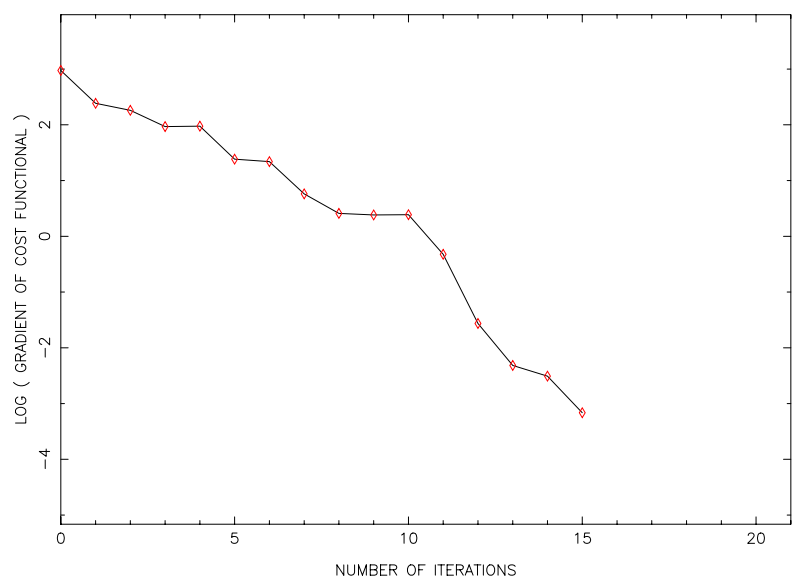

(d)

Fig. 19. Evolution of the cost functional and gradient norm during LBFGS minimization with the artificial viscosity model for time $=0.15$ (first set of observations $(\mathrm{a}, \mathrm{b})$ and second set of observations $(\mathrm{c}, \mathrm{d}))$. 


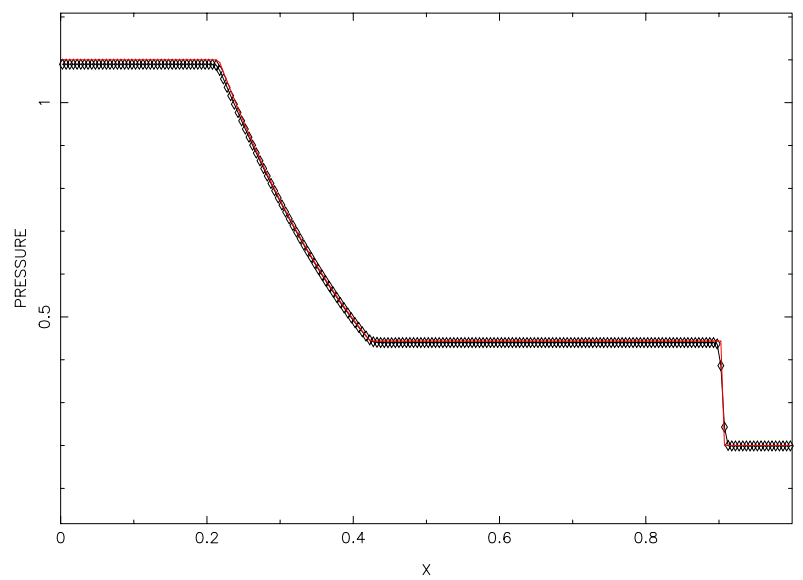

(a)

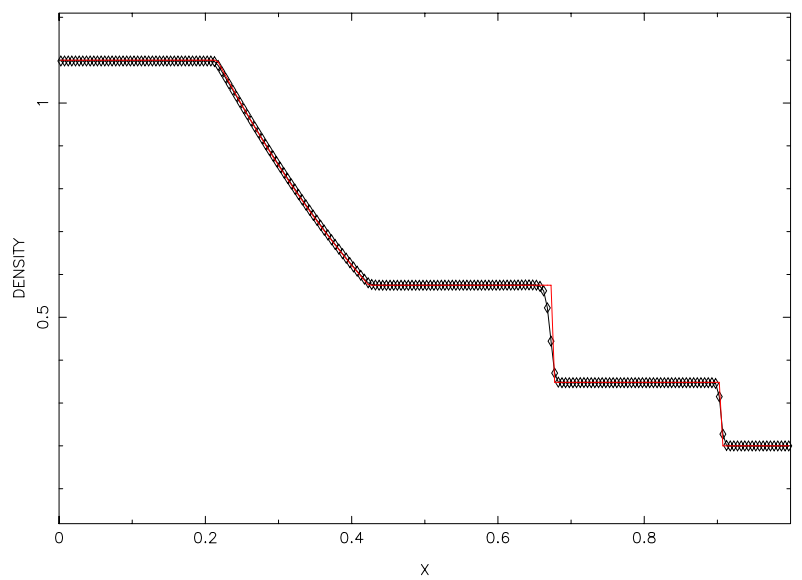

(b)

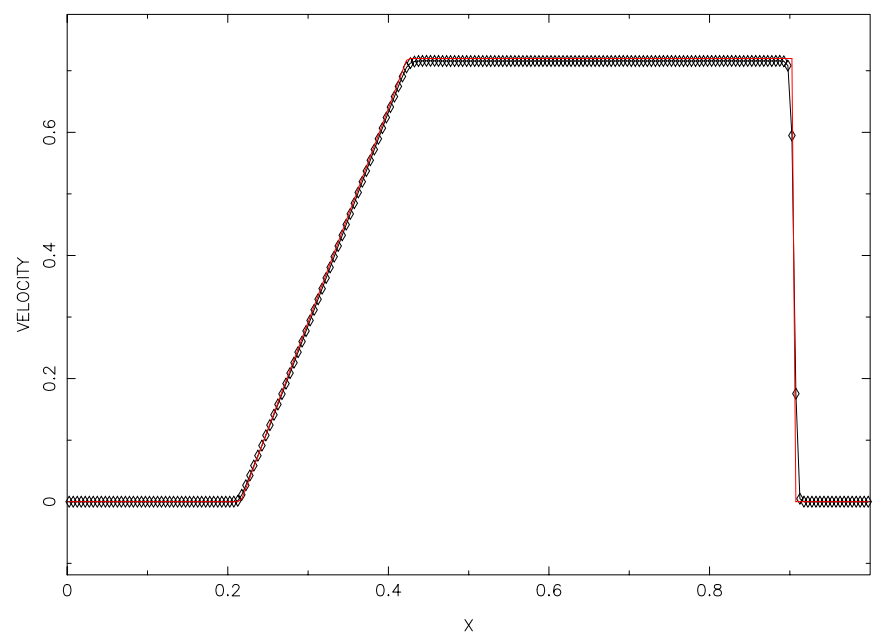

(c)

Fig. 20. Desired solution (first set of observations) (red line) and numerical solution $(\diamond)$ after nonsmooth optimization of high-resolution model at time $=0.24$ for a) pressure, b) density and c) velocity. 


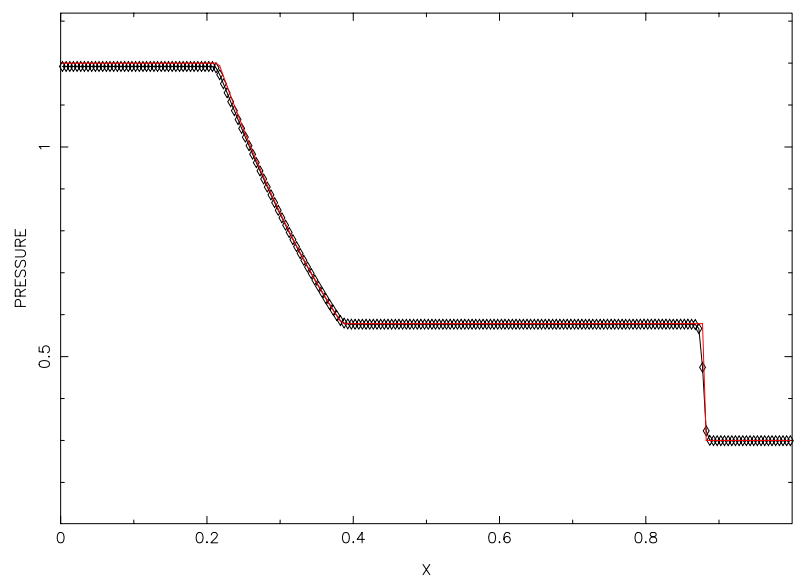

(a)

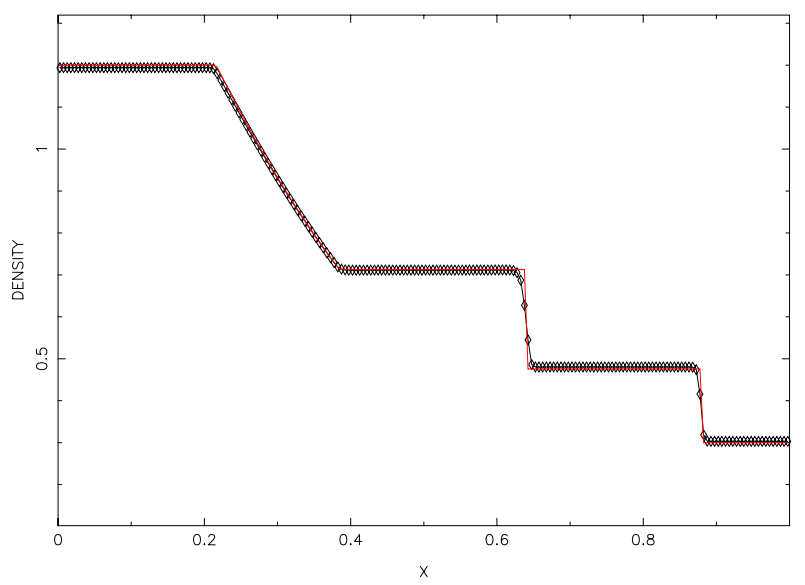

(b)

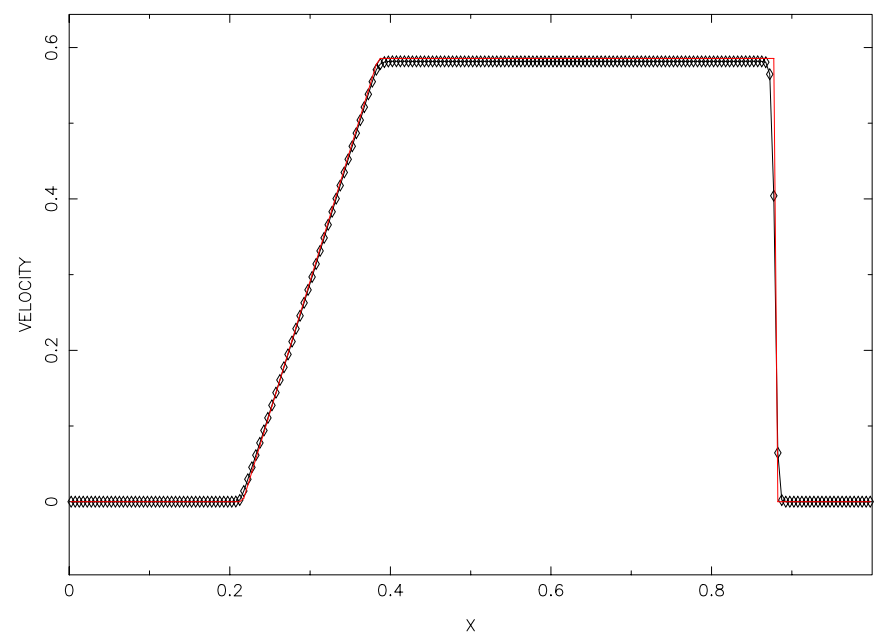

(c)

Fig. 21. Desired solution (second set of observations) (red line) and numerical solution $(\diamond)$ after nonsmooth optimization of high-resolution model at time $=0.24$ for a) pressure, b) density and c) velocity. 


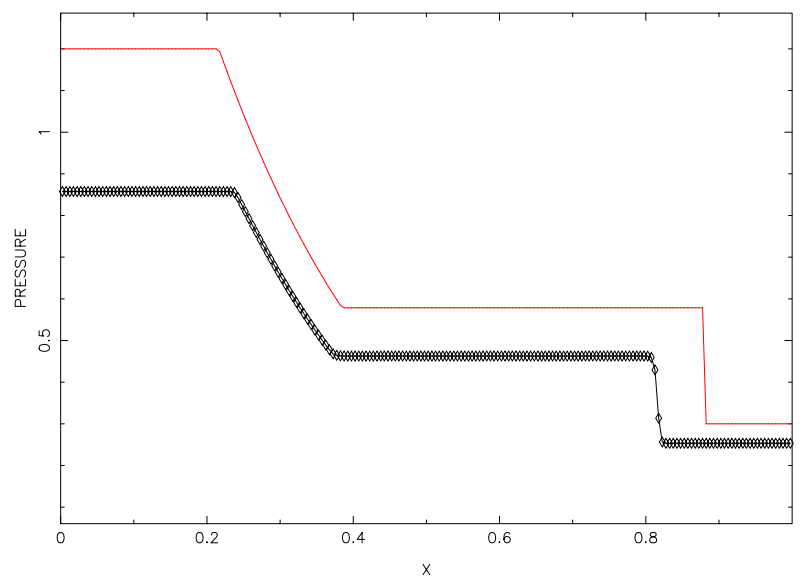

(a)

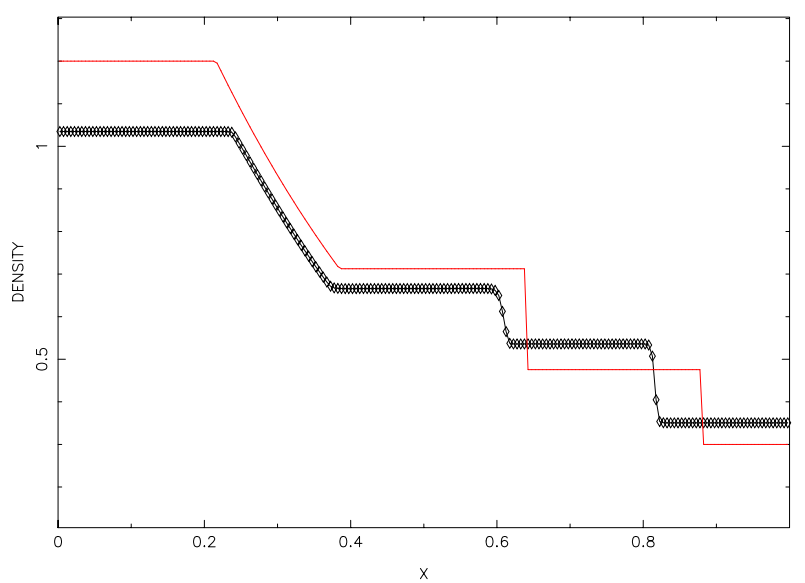

(b)

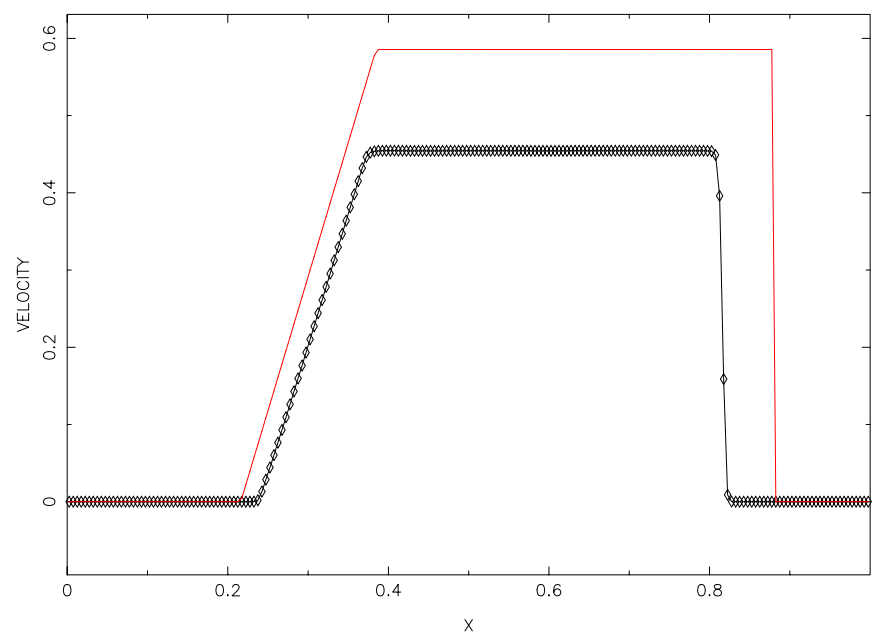

(c)

Fig. 22. Desired solution (second set of observations) (red line) and numerical solution $(\diamond)$ after LBFGS optimization of high-resolution model at time $=0.24$ for a) pressure, b) density and c) velocity. 


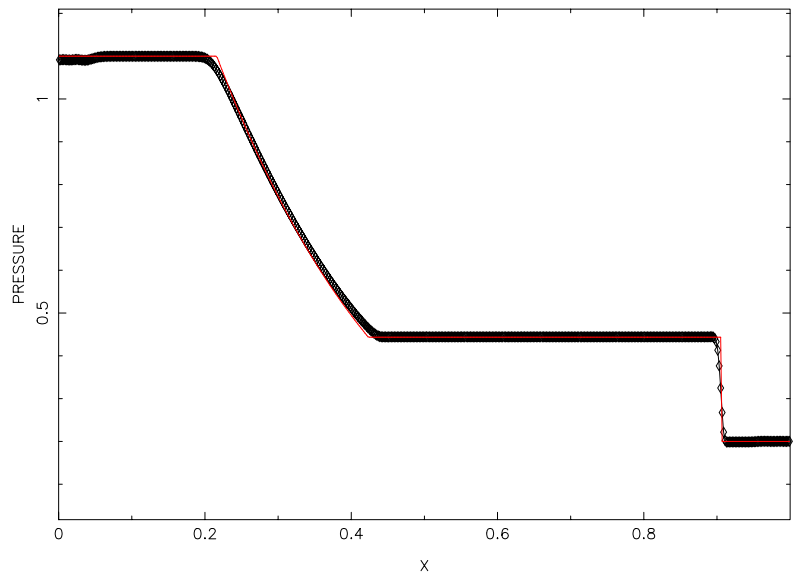

(a)

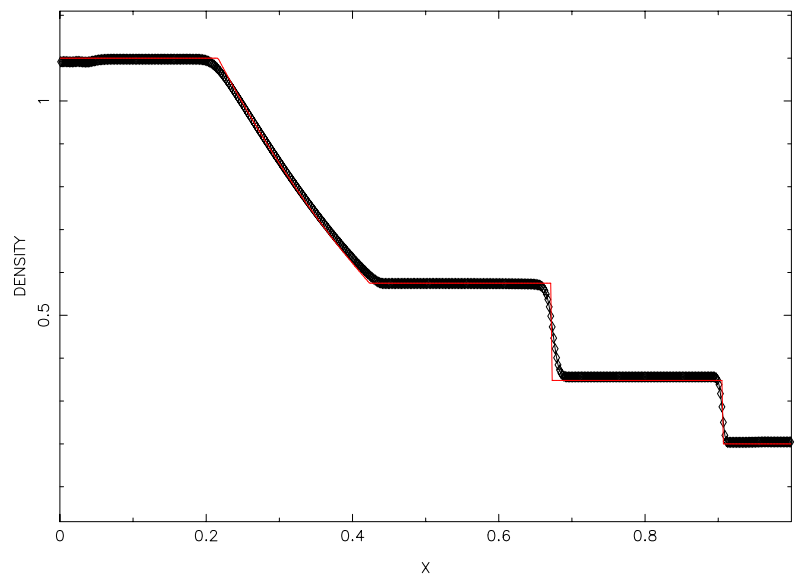

(b)

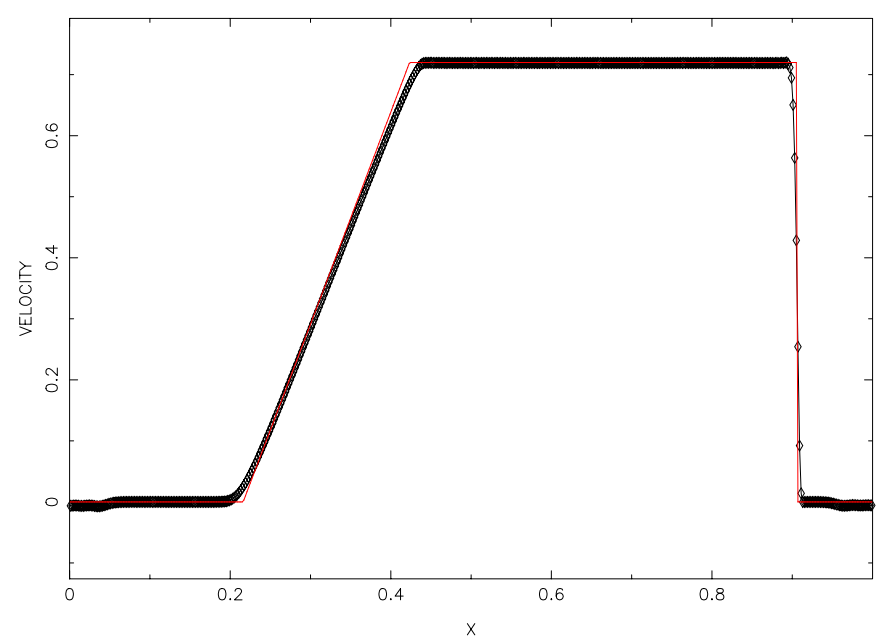

(c)

Fig. 23. Desired solution (first set of observations) (red line) and numerical solution $(\diamond)$ after nonsmooth optimization of artificial velocity model at time $=0.24$ for a) pressure, b) density and c) velocity. 


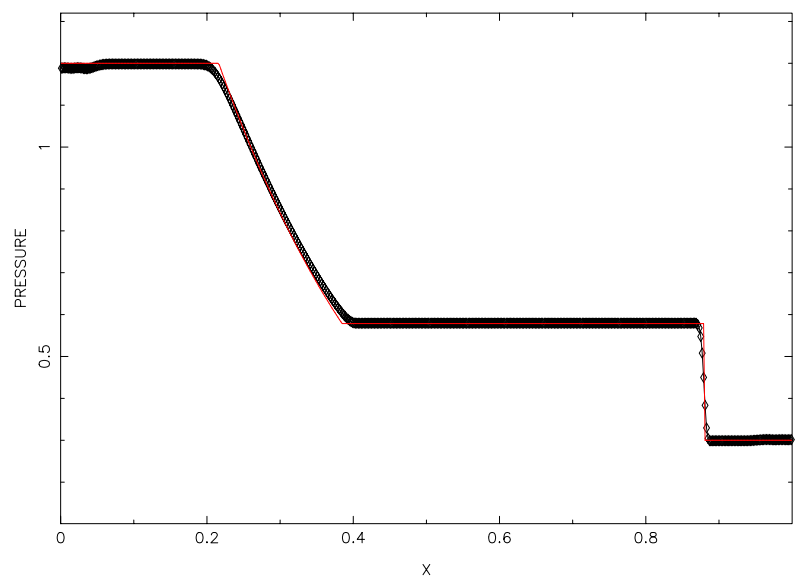

(a)

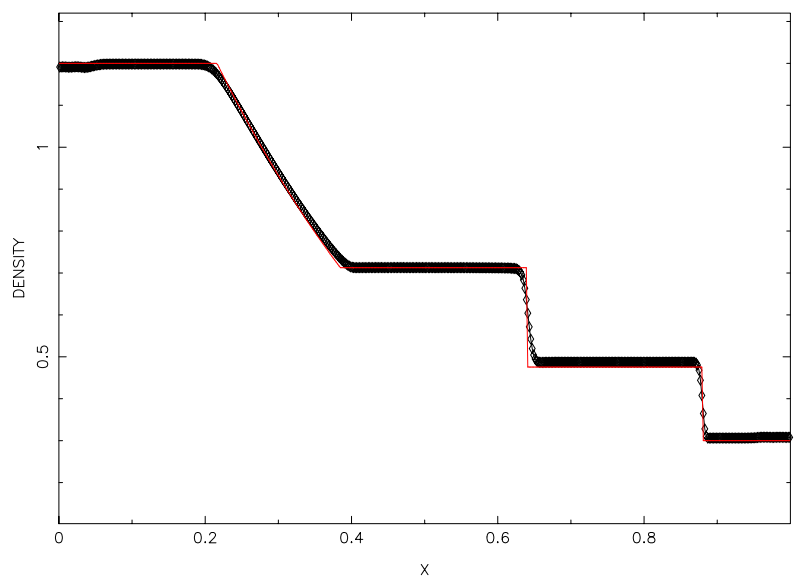

(b)

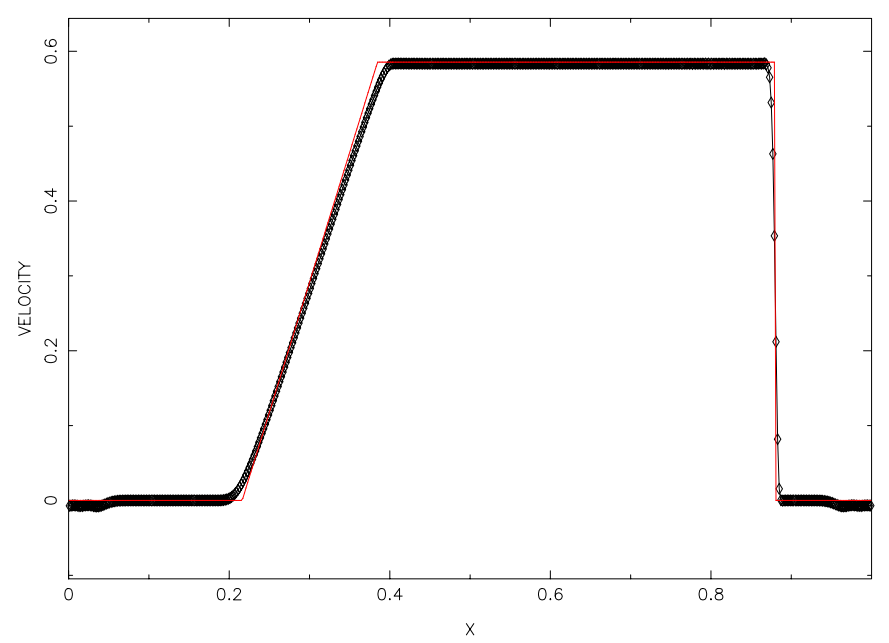

(c)

Fig. 24. Desired solution (second set of observations) (red line) and numerical solution $(\diamond)$ after nonsmooth optimization of artificial velocity model at time $=0.24$ for a) pressure, b) density and c) velocity. 


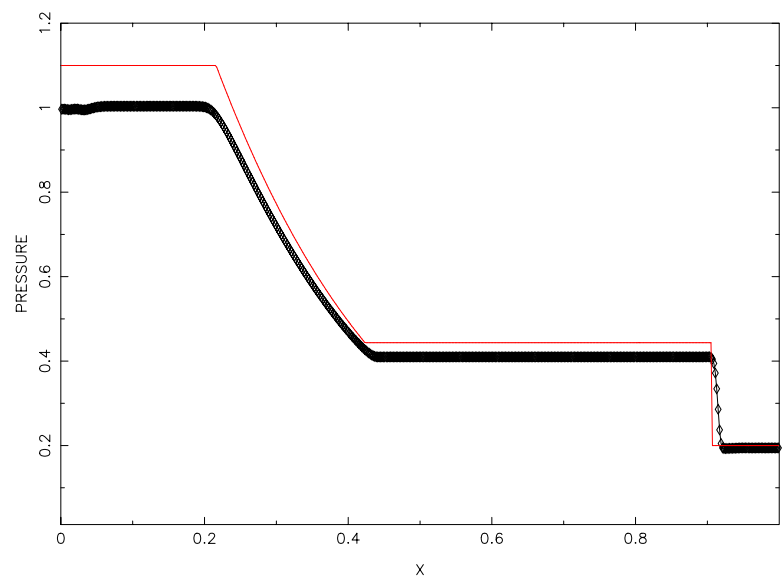

(a)

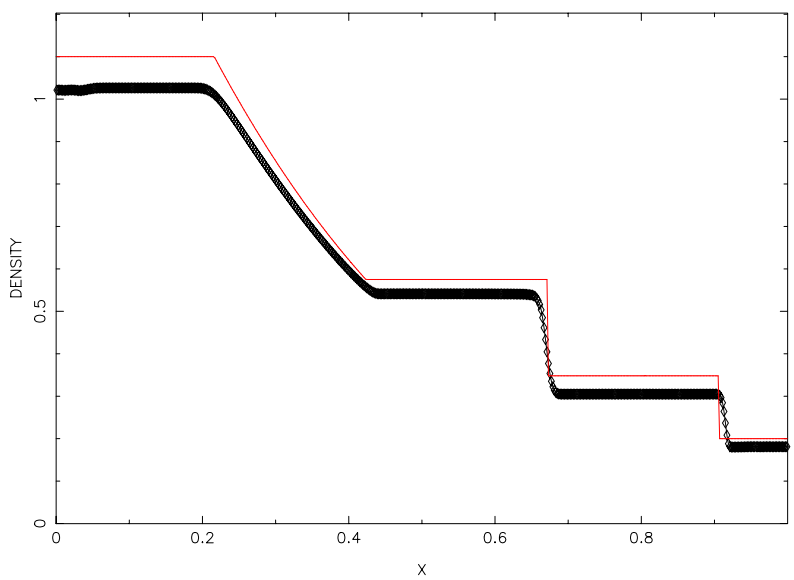

(b)

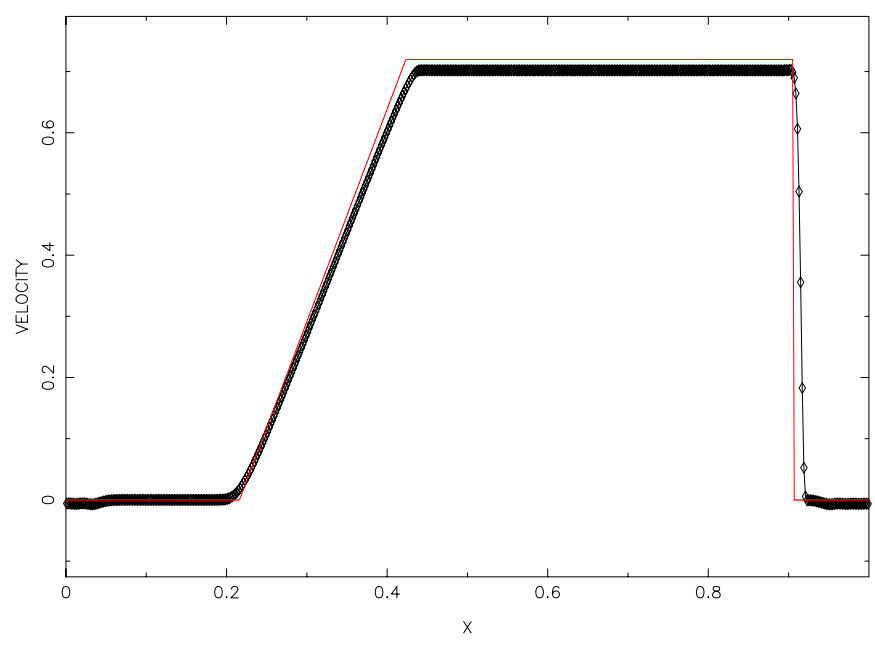

(c)

Fig. 25. Desired solution (first set of observations) (red line) and numerical solution $(\diamond)$ after LBFGS optimization of artificial velocity model at time $=0.24$ for a) pressure, b) density and c) velocity. 


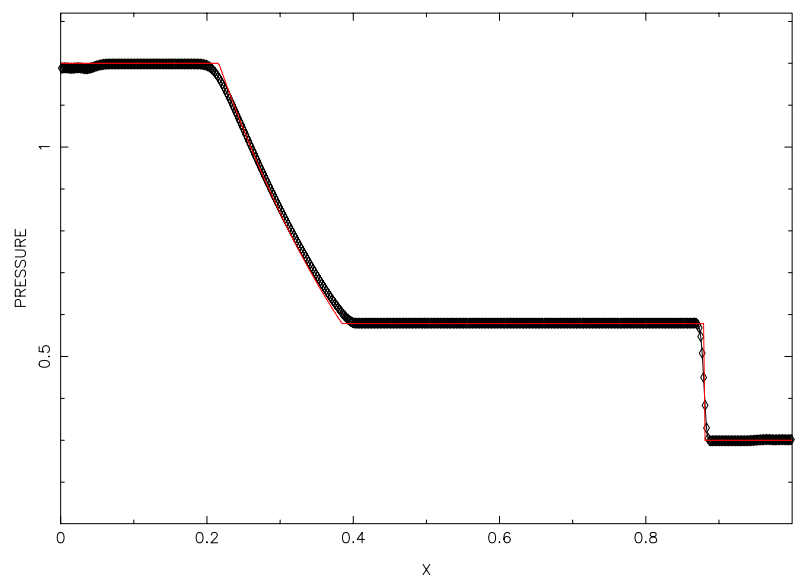

(a)

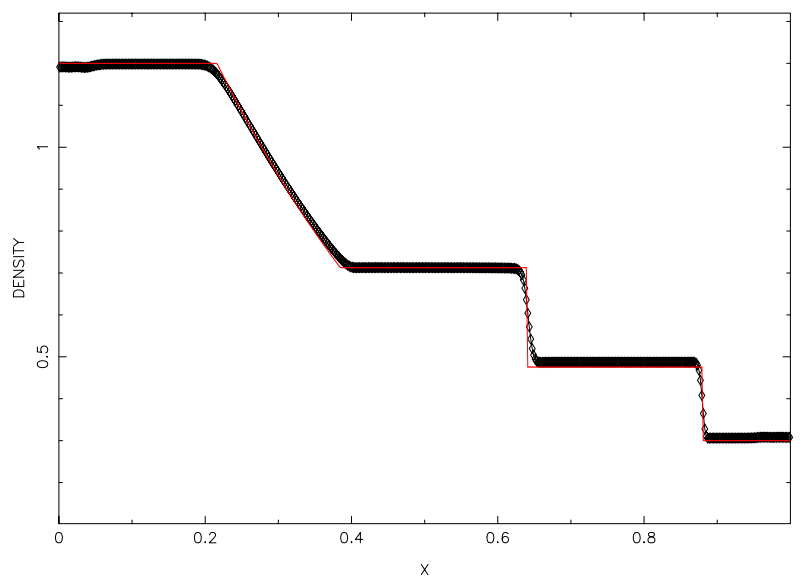

(b)

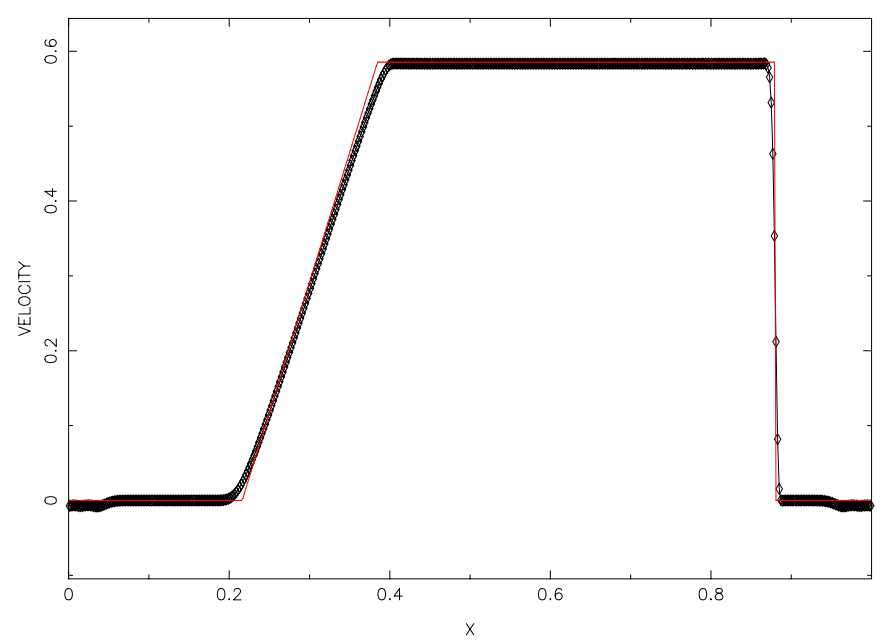

(c)

Fig. 26. Desired solution (second set of observations) (red line) and numerical solution $(\diamond)$ after LBFGS optimization of artificial velocity model at time $=0.24$ for a) pressure, b) density and c) velocity. 


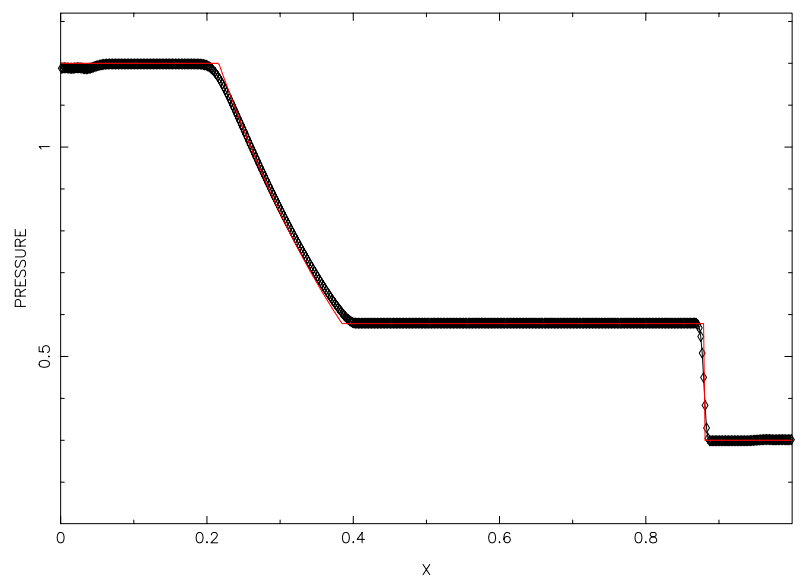

(a)

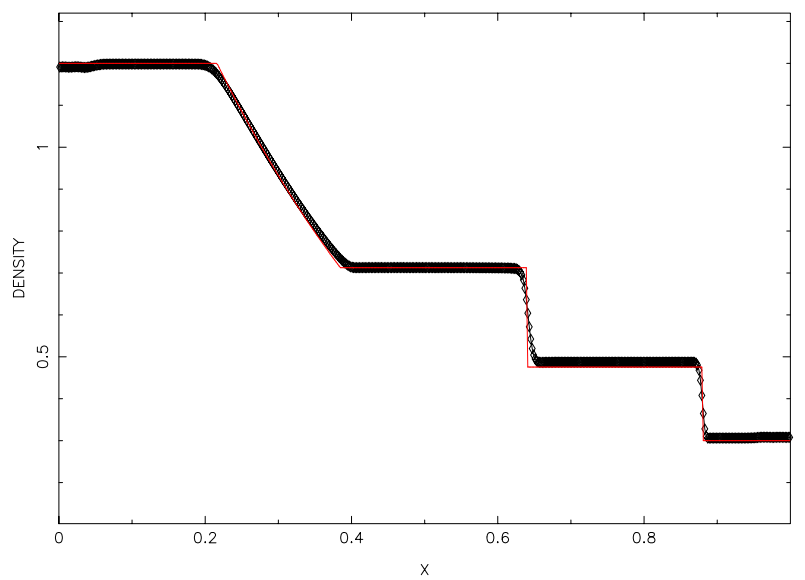

(b)

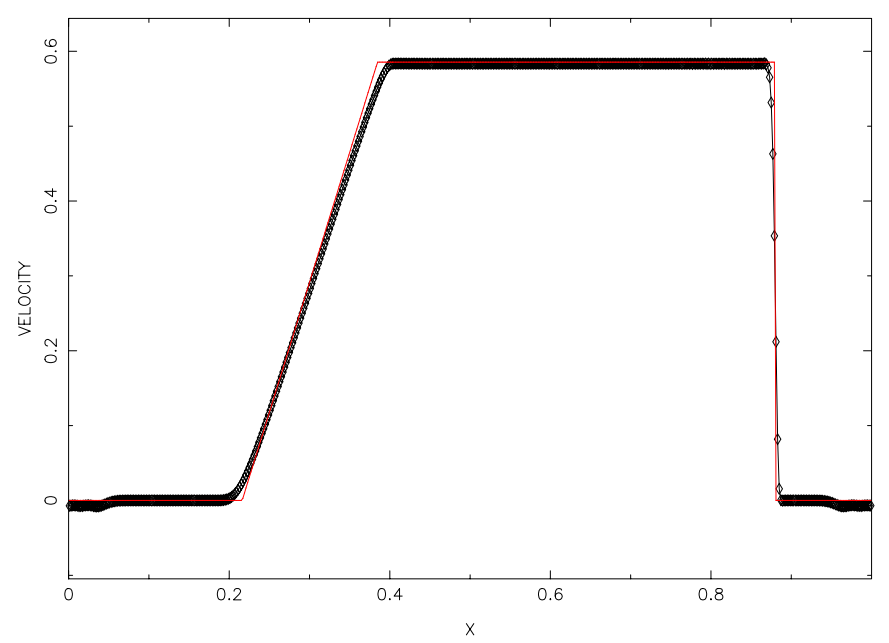

(c)

Fig. 27. Desired solution (first set of observations) (red line) and numerical solution $(\diamond)$ after LBFGS optimization (with weight=0.0) of artificial velocity model at time $=0.24$ for a) pressure, b) density and c) velocity. 


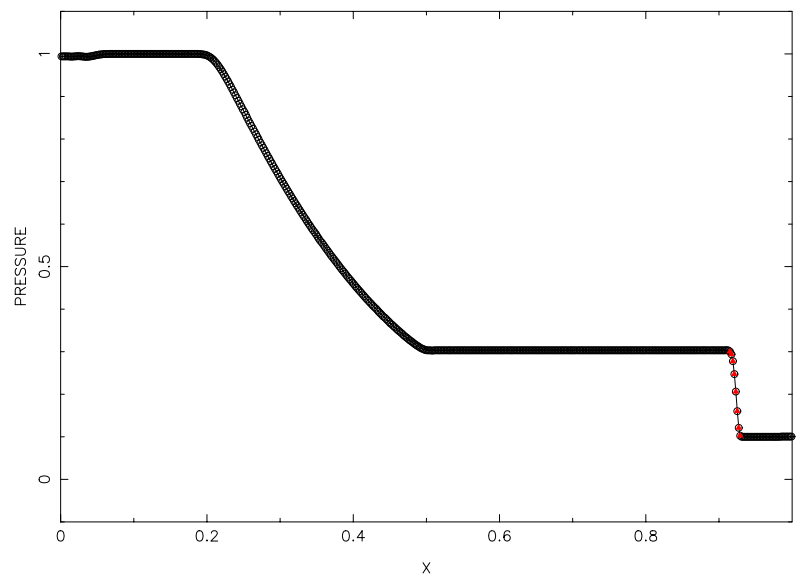

(a)

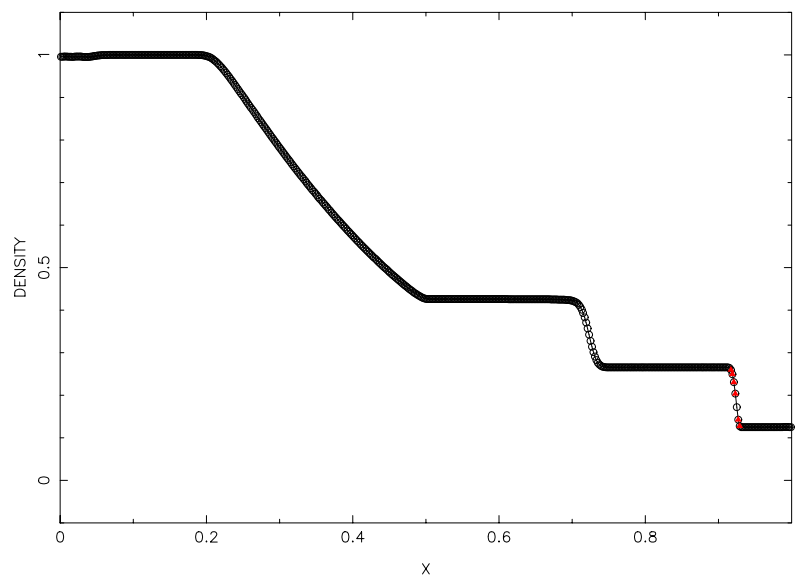

(b)

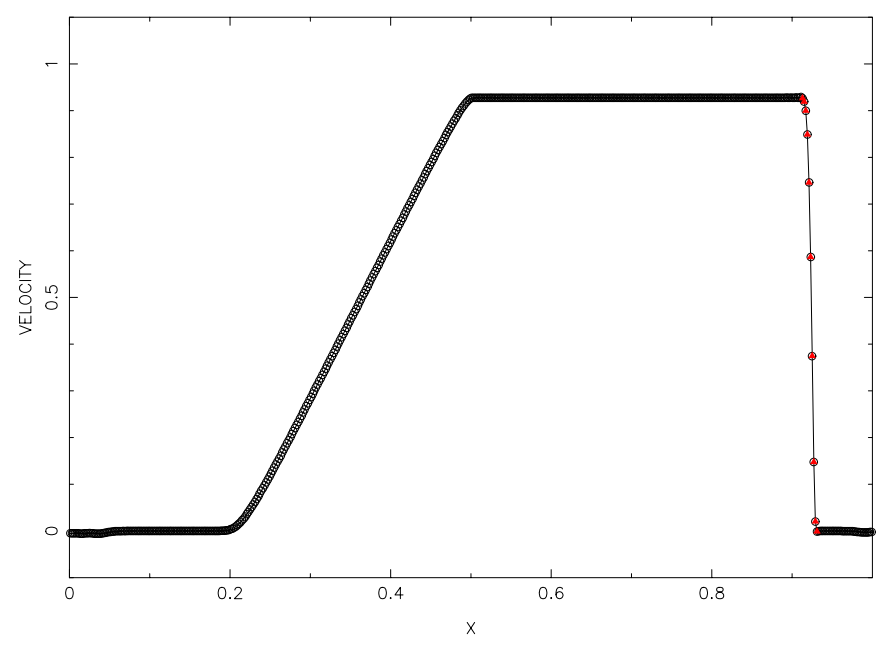

(c)

Fig. 28. The result of finding discontinuities (red filled triangles) for the numerical solution of artificial velocity model at time $=0.24$ for a) pressure, b) density and c) velocity. 


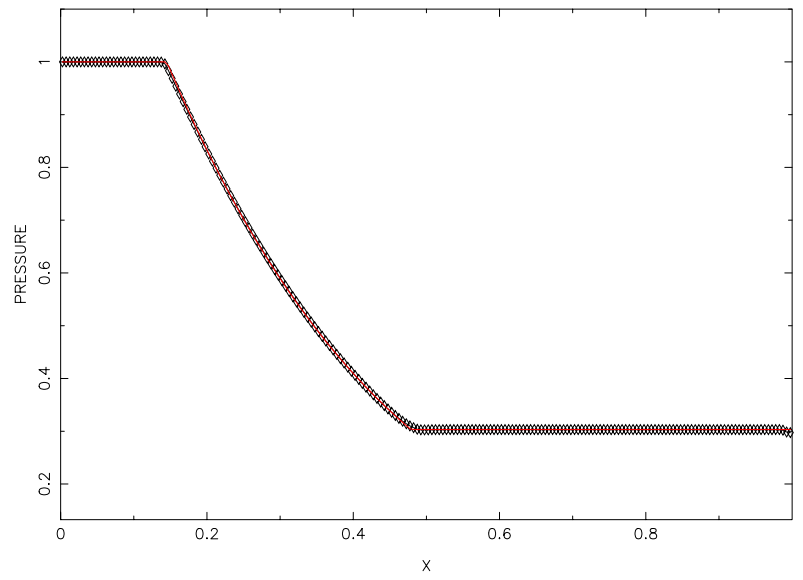

(a)

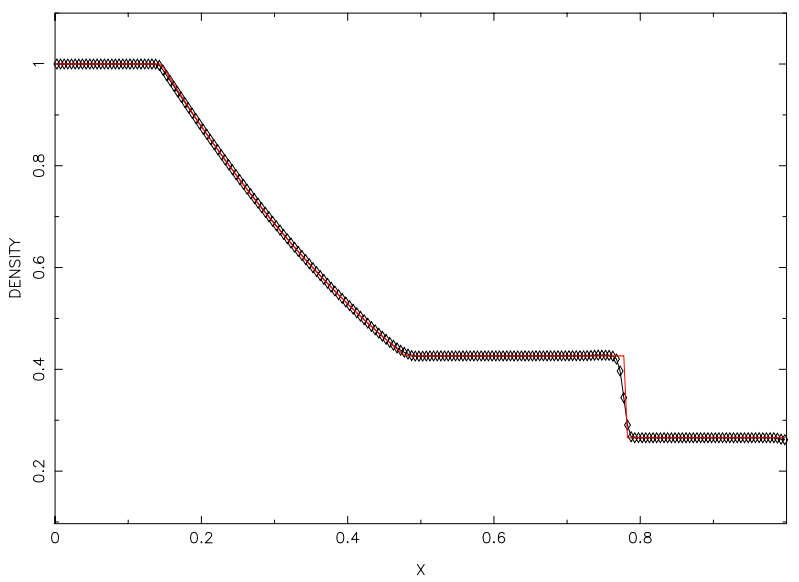

(b)

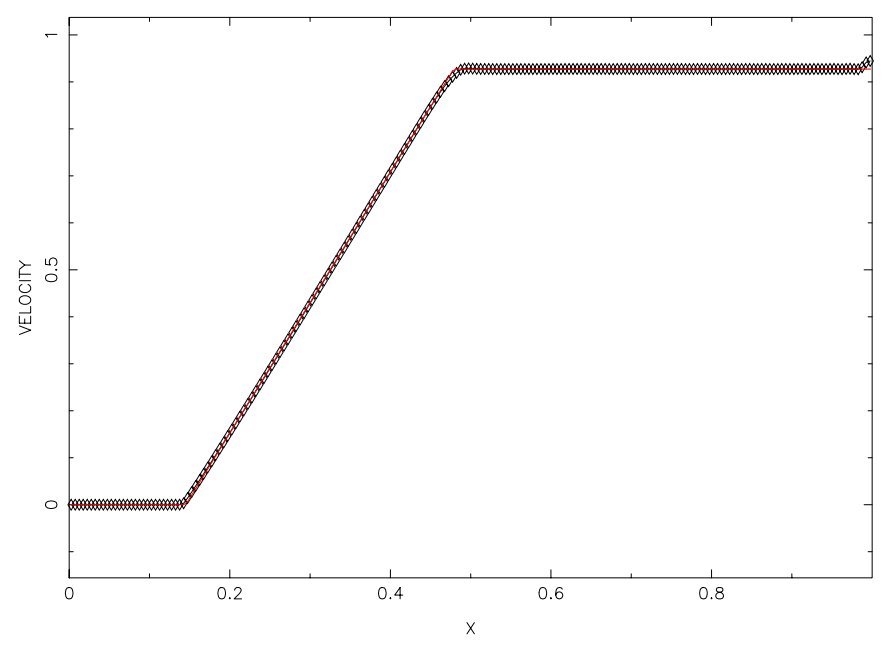

(c)

Fig. 29. Numerical ( $\diamond)$ and analytical (red line) solution of high-resolution model for the shock-tube problem at time $=0.30$ for a) pressure, b) density and c) velocity. 

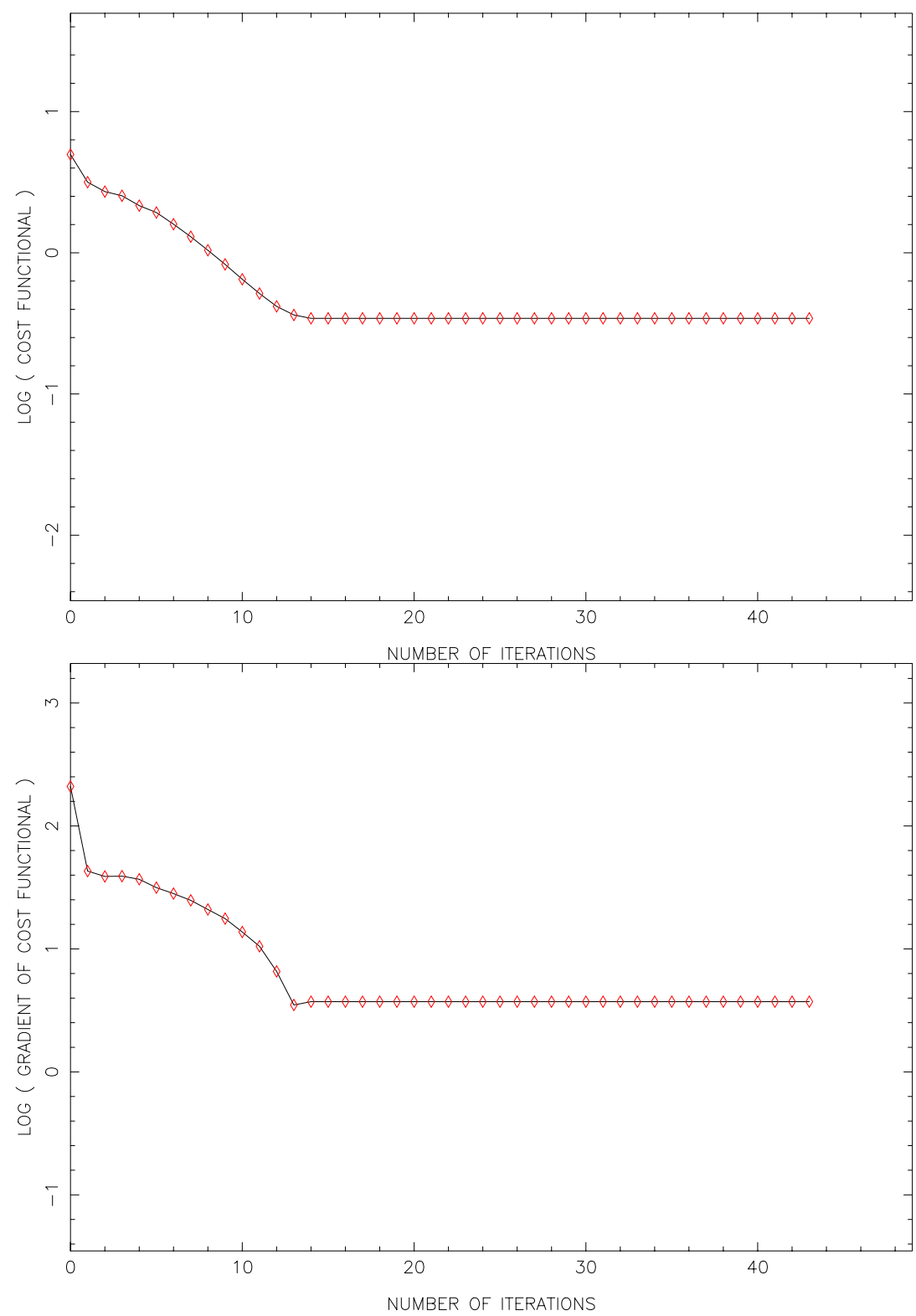

Fig. 30. Evolution of the cost functional and gradient norm during nonsmooth minimization with the initial guess from the LBFGS output for the artificial viscosity model for time $=0.24$ and first set of observations . 